\title{
Production of cold molecules via magnetically tunable Feshbach resonances
}

\author{
Thorsten Köhler and Krzysztof Góral
}

Clarendon Laboratory, Department of Physics, University of Oxford, Parks Road, Oxford, OX1 3PU, United Kingdom

Paul S. Julienne

Atomic Physics Division, National Institute of Standards and Technology, 100 Bureau Drive Stop 8423, Gaithersburg, Maryland 20899-8423

(Dated: July 16, 2018)

\begin{abstract}
Magnetically tunable Feshbach resonances were employed to associate cold diatomic molecules in a series of experiments involving both atomic Bose as well as two spin component Fermi gases. This review illustrates theoretical concepts of both the particular nature of the highly excited Feshbach molecules produced and the techniques for their association from unbound atom pairs. Coupled channels theory provides the rigorous formulation of the microscopic physics of Feshbach resonances in cold gases. Concepts of dressed versus bare energy states, universal properties of Feshbach molecules, as well as the classification in terms of entrance- and closed-channel dominated resonances are introduced on the basis of practical two-channel approaches. Their significance is illustrated for several experimental observations, such as binding energies and lifetimes with respect to collisional relaxation. Molecular association and dissociation are discussed in the context of techniques involving linear magnetic field sweeps in cold Bose and Fermi gases as well as pulse sequences leading to Ramsey-type interference fringes. Their descriptions in terms of Landau-Zener, two-level mean field as well as beyond mean field approaches are reviewed in detail, including the associated ranges of validity.
\end{abstract}

\section{Contents}

I. Introduction

II. Weakly bound diatomic molecules

III. Feshbach resonances

A. Molecular physics of resonances

1. Inter-atomic interactions

2. Coupled channels scattering

3. Threshold collisions

4. Example of channel classification

5. Near threshold bound states

B. Two-channel approach

1. Two-channel Hamiltonian

2. The bare Feshbach resonance state

C. Dressed energy states

1. Dressed continuum states

2. Single resonance approach

3. Width and shift of a zero energy resonance

4. Dressed molecular bound states

D. Universal properties of Feshbach molecules

1. Universal binding energy

2. The Wigner threshold law

3. Closed channel admixture

E. Experimental signatures of universality

1. Collisional relaxation

2. Lifetime of Feshbach molecules in Fermi gases

3. Lifetime of Feshbach molecules in Bose gases

F. Classification of zero energy resonances

1. Size of the universal regime

2. Entrance-channel dominated resonances

G. Characteristic parameters of zero energy resonances

1. The background scattering potential

2. The resonance energy

3. Inter-channel coupling

IV. Association of Feshbach molecules

A. Linear sweeps of the magnetic field strength

1. Adiabatic association of Feshbach molecules

2. Exact time evolution of a single atom pair

3. Landau-Zener approach

B. Magnetic field sweeps in Bose-Einstein condensates
1. Limit of high ramp speeds

2. Two-level mean field approach

C. Molecule production in cold Bose and Fermi gases

1. Transitions from continuum to bound states

2. Fast sweep limit of molecule production

3. Saturation of molecule production

D. Dissociation of Feshbach molecules

\section{Atom-molecule coherence}

A. Ramsey interferometry with atoms and molecules

1. Magnetic field pulse sequence

2. Dynamics of a single atom pair

B. Number of dimers produced in Bose and Fermi gases

C. Dynamics of partially condensed Bose gases

1. Beyond mean field approaches

2. The remnant Bose-Einstein condensate

3. Feshbach molecule and burst components

4. Three-component Ramsey fringes

VI. Conclusions and Outlook

Acknowledgements

References

\section{INTRODUCTION}

Resonances in general refer to the energy dependent enhancement of inter-particle collision cross sections due to the existence of a meta-stable state. Since the beginning of quantum mechanics such phenomena have been the subject of numerous studies in nuclear (Blatt and Weisskopf, 1952) as well as atomic and molecular physics (Bransden and Joachain, 2003; Child, 1974). The meta-stable state may be described in terms of tunnelling across a barrier of the potential energy or coupling of a bound level of a subsystem to its environment (Fano, 1935, 1961; Feshbach, 1958, 1962; Rice, 1933). These scenarios are respectively referred to as shape and Feshbach resonances. 
In the context of cold atomic gases, collision phenomena associated with Feshbach resonances were predicted first for systems of spin polarised hydrogen and deuterium (Stwallev, 1976; Uang and Stwalley, 1980) as well as lithium (Uang et al., 1981) in the presence of magnetic fields. The associated resonance energies depend on the field strength via the Zeeman effect in the hyperfine levels. This research has gained substantially new experimental perspectives since the achievement of Bose-Einstein condensation (Bose, 1924; Einstein, 1924, 1925) of dilute vapours of alkali atoms (Anderson et al., 1995; Bradley et al., 1997, 1995; Davis et al., 1995). Contrary to conventional gases, such atomic clouds with densities five orders of magnitude less than air and sub $\mu \mathrm{K}$ temperatures give rise to binary collision energies close to the threshold between scattering and molecular binding. In this extraordinary regime, magnetically tunable Feshbach resonances can be employed to manipulate the inter-atomic forces determined by the scattering length (Tiesinga et al., 1993), as well as for the production of diatomic molecules at rest (Timmermans et al., 1999b).

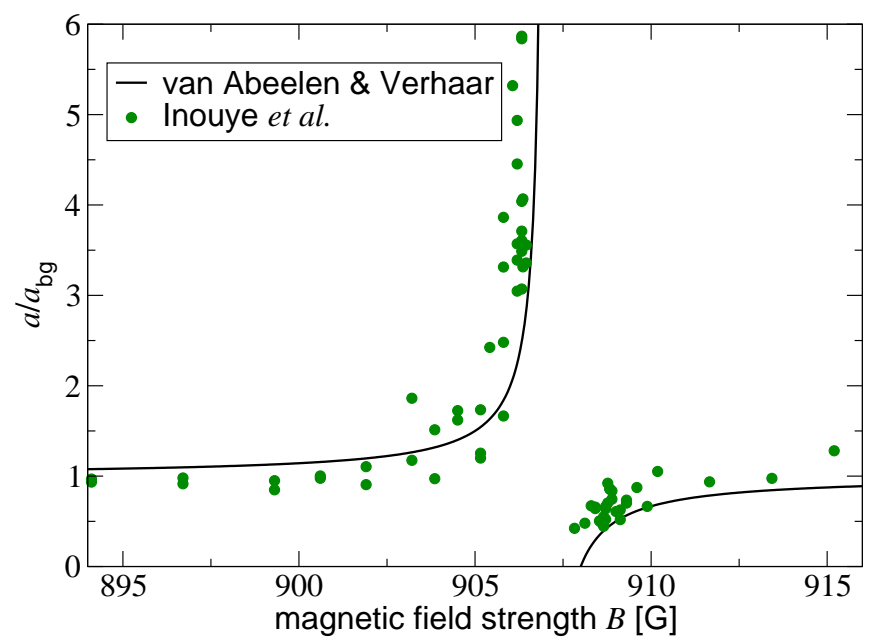

FIG. 1 (Colour in online edition) Order of magnitude variation of the scattering length, $a$, as a function of the magnetic field strength, $B$, in the vicinity of the $907 \mathrm{G}(90.7 \mathrm{mT})$ zero energy resonance of ${ }^{23} \mathrm{Na}$. The circles refer to measurements using sodium Bose-Einstein condensates (Inouve et al., 1998), while the solid curve indicates associated theoretical predictions (van Abeelen and Verhaar, 1998). The scattering length is normalised to its asymptotic value $a_{\mathrm{bg}}$ away from the singularity at $B_{0}=907 \mathrm{G}$. Adapted by permission from Macmillan Publishers Ltd: Nature (London), (Inouye et al., 1998), copyright (1998).

Both techniques were demonstrated in several series of experiments with widespread applications throughout the physics of cold gases. Figure 1 illustrates the manipulation of the scattering length, $a$, in a ${ }^{23} \mathrm{Na}$ Bose-Einstein condensate exposed to a spatially homogeneous magnetic field of variable strength, $B$ (Inouye et al., 1998). The pole in Fig. 1 at about $907 \mathrm{G}^{1}$ is due to the near degeneracy of the en-

\footnotetext{
${ }^{1}$ The Standard International unit for magnetic field is Tesla, whereas most
}

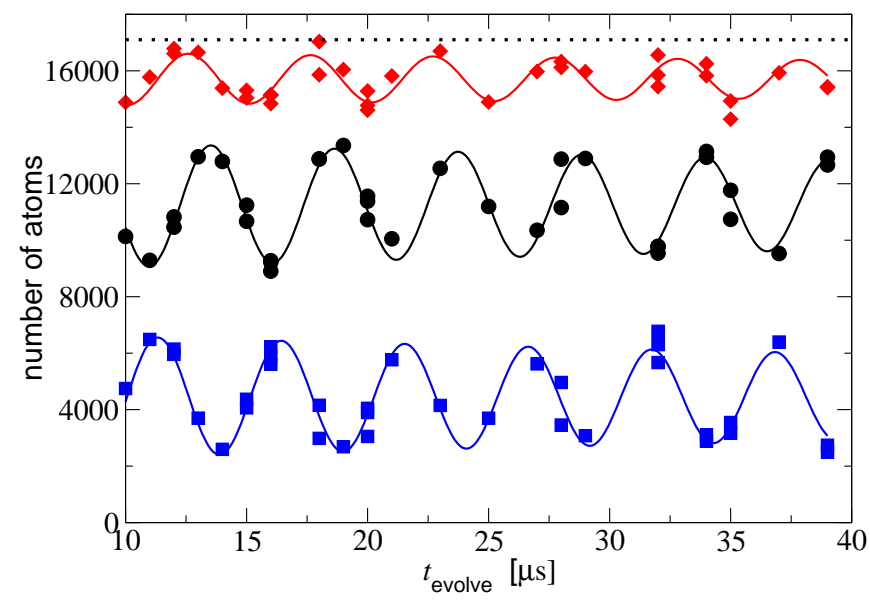

FIG. 2 (Colour in online edition) Ramsey fringes between atomic and molecular components produced from a Bose-Einstein condensate of $17100{ }^{85} \mathrm{Rb}$ atoms (Donley et al., 2002) via a sequence of magnetic field pulses in the vicinity of the $155 \mathrm{G}$ zero energy resonance. Circles and squares refer to the number of particles in the remnant condensate and in an atomic burst consisting of correlated pairs with a comparatively high relative velocity, respectively. Diamonds indicate the total amount of particles in both of these components. Its difference with respect to the total number of atoms (dotted line) indicates the production of undetected Feshbach molecules. The fringes are shown as a function of the delay time of the interferometer, $t_{\text {evolve }}$, in which the atomic and molecular states acquire their phase difference. The fringe frequency provides an accurate measure of the binding energy (Claussen et al., 2003). Adapted by permission from Macmillan Publishers Ltd: Nature (London), (Donley et al., 2002), copyright (2002).

ergy associated with a Feshbach resonance and the threshold. Such a singularity, usually referred to as a zero energy resonance (Taylor, 1972) $)^{2}$, allows the scattering length, in principle, to assume all values between $-\infty$ and $\infty$. Cold gases with such widely tunable interactions were subsequently realised for several species of alkali atoms, such as ${ }^{85} \mathrm{Rb}$ (Courteille et al., 1998; Roberts et al., 1998) and ${ }^{133} \mathrm{Cs}$ (Chin et al., 2003; Vuletic et al., 1998). Their applications involve the Bose-Einstein condensation of ${ }^{85} \mathrm{Rb}$ (Cornish et al., 2000) and ${ }^{133} \mathrm{Cs}$ (Weber et al., 2003) as well as studies of the collapse of condensates with negative scattering lengths (Donley et al., 2001; Roberts et al., 2000a).

Quite generally, the zero energy resonance position exactly coincides with the field strength at which the energy of a diatomic vibrational bound state becomes degenerate with the threshold for dissociation into an atom pair at rest. On the side of positive scattering lengths, this Feshbach molecular state describes a stable molecule in the absence of background gas collisions which ceases to exist at the position of the singularity. In the context of two-body systems involving the

of the papers quoted in this review use Gauss as the unit. Consequently, we use $\mathrm{G}$ here, where $1 \mathrm{G}=10^{-4} \mathrm{~T}$.

${ }^{2}$ In the context of cold gases, a singularity of the scattering length is also often referred to simply as a Feshbach resonance. 
same species of atoms such a bound state is usually referred to as a dimer. The relation between collision resonances above threshold and bound states below it links the manipulation of interactions to the molecular conversion of separated atom pairs.

Production of cold dimers was demonstrated first (Fioretti et al., 1998; Nikolov et al., 1999; Takekoshi et al., 1999; Wynar et al., 2000) via photo-association of atoms (Weiner et al., 1999). This achievement was followed by studies of condensed gases of ${ }^{85} \mathrm{Rb}$ exposed to time dependent magnetic field variations consisting of pairs of pulses in the vicinity of the $155 \mathrm{G}$ zero energy resonance (Donley et al., 2002). These experiments temporarily probed the regime of strong interactions where the size of the scattering length was comparable to the average inter-atomic distance. Such perturbations led to the three distinct components of the gas illustrated in Fig. 2] The oscillatory behaviour of their occupations as a function of the time delay between the pulses implied an interpretation in terms of Ramsey interference fringes due to a superposition state of separated atoms and Feshbach molecules.
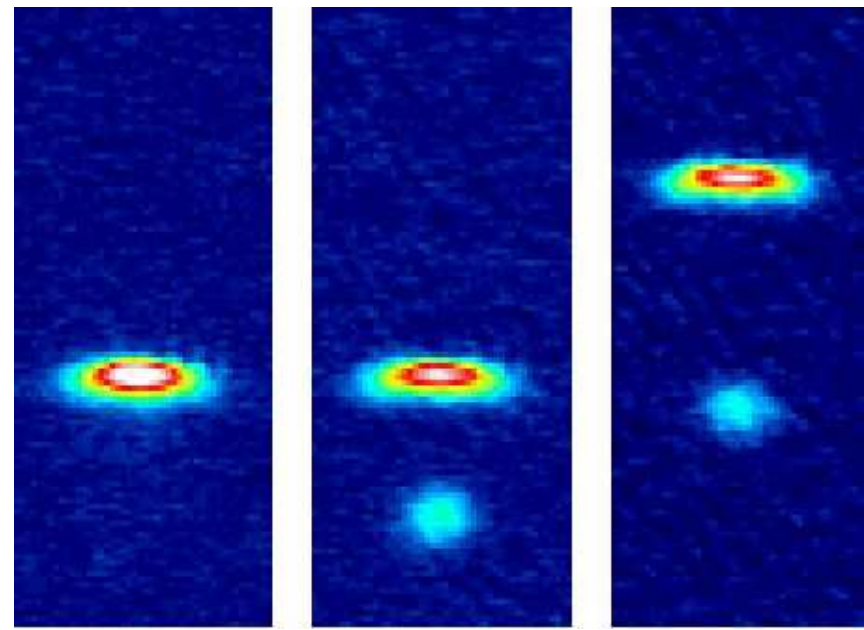

FIG. 3 (Colour in online edition) A molecular component of about 3000 dimers is out-coupled from a dilute cloud of 25000 ground state cesium atoms using Stern-Gerlach separation in an inhomogeneous magnetic field (Herbig et al., 2003). The left and middle images refer to situations in which the magnetic field is calibrated in such a way that it exactly compensates for the gravitational force acting on the atoms in gases without and with a molecular component, respectively. Due to their magnetic moment difference of $-0.57 \mu_{\text {Bohr }}$ ( $\mu_{\text {Bohr }}=9.27400949 \times 10^{-24} \mathrm{~J} / \mathrm{T}$ is the Bohr magneton) with respect to separated atoms, the Feshbach molecules in the middle image drop down from the atomic cloud which is levitated and centred at the same position as in the left reference image. Conversely, the right image shows levitation of molecules and upward acceleration of the separated atoms using a suitably adjusted inhomogeneous magnetic field.

According to Fig. 2, the pulse sequence allowed a conversion of up to about $16 \%$ of the ${ }^{85} \mathrm{Rb}$ atoms into Feshbach molecules. Subsequent experiments improved the production efficiency by using magnetic field sweeps from negative to positive scattering lengths across a zero energy res- onance. This technique was applied to cold gases consisting of incoherent two spin component mixtures of either ${ }^{40} \mathrm{~K}$ or ${ }^{6} \mathrm{Li}$ atoms (Cubizolles et al., 2003; Jochim et al., 2003a; Regal et al., 2003a; Strecker et al., 2003) as well as BoseEinstein condensates of ${ }^{133} \mathrm{Cs},{ }^{87} \mathrm{Rb}$ and ${ }^{23} \mathrm{Na}$ (Dürr et al., 2004a; Herbig et al., 2003; Xu et al., 2003). In the context of these experiments, new schemes for the detection of Feshbach molecules were developed. These techniques involve radio frequency (rf) photo-dissociation, atom loss and recovery, as well as the spatial separation of molecules from the remnant atomic cloud followed by their dissociation using magnetic field sweeps. Separation of Feshbach molecules from an atomic gas, for instance, may be achieved via the SternGerlach approach of Fig. 3 (Chin et al., 2005; Dürr et al., 2004a; Herbig et al., 2003), probing the magnetic moments of dimers at magnetic fields away from the zero energy resonance.
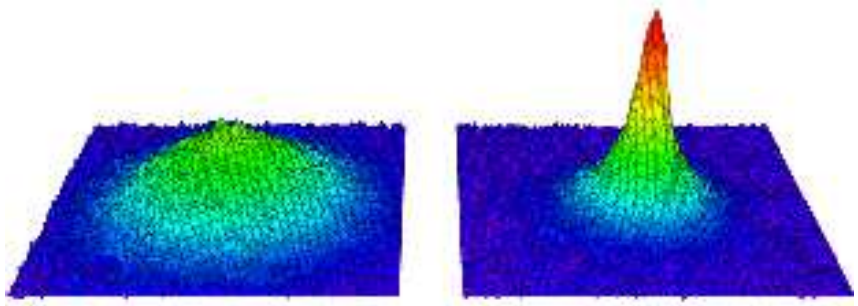

FIG. 4 (Colour in online edition) Density distributions of dilute thermal (left image) and partially Bose-Einstein condensed (right image) gases of ${ }^{40} \mathrm{~K}_{2}$ Feshbach molecules (Greiner et al., 2003). Both molecular vapours were produced using the technique of linear magnetic field sweeps across the $202 \mathrm{G}$ zero energy resonance. The initial two spin component clouds of 470000 and 250000 Fermi atoms were prepared above $(250 \mathrm{nK})$ and below $(79 \mathrm{nK})$ the associated critical temperatures for condensation, respectively. The condensate fraction in the right image is $12 \%$. Adapted by permission from Macmillan Publishers Ltd: Nature (London), (Greiner et al., 2003), copyright (2003).

Near resonance, their large spatial extent associated with a loose bond (Köhler et al., 2003a b) can lead to a remarkable stability of Feshbach molecules with respect to inelastic collisions in the environment of a two spin component Fermi gas (Petrov et al., 2004, 2005b). The lifetimes of such dimers, ranging from about $100 \mathrm{~ms}$ in the case of ${ }^{40} \mathrm{~K}_{2}$ (Regal et al., 2004a) to several seconds in ${ }^{6} \mathrm{Li}_{2}$ gases (Cubizolles et al., 2003; Jochim et al., 2003a), were sufficient for the observation of their Bose-Einstein condensation. This achievement was based on either the magnetic field sweep technique illustrated in Fig. 4 (Greiner et al., 2003) or evaporative cooling of a molecular cloud (Jochim et al., 2003b; Zwierlein et al., 2003). Such pioneering experiments gave rise to an ongoing series of studies (Bartenstein et al., 2004; Bourdel et al., 2004; Chin et al., 2004a; Kinast et al., 2004; Loftus et al., 2002; O’Hara et al., 2002; Partridge et al., 2005; Regal et al., 2004b; Zwierlein et al., 2005, 2004) probing the crossover from Bardeen-Cooper-Schrieffer (BCS) pairing at negative scattering lengths to molecular Bose-Einstein condensation (Eagles, 1969; Leggett, 1980; Nozières and Schmitt-Rink, 1985; Randeria, 1995). 
This article reviews the theoretical background of the exotic species of highly excited Feshbach molecules produced as well as their formation in the environment of a cold gas.

Section 【 briefly introduces the concept of universality of weakly bound dimers, their relevant length and energy scales as well as the general form of their wave functions. This discussion integrates near resonant Feshbach molecules into the general class of quantum halo systems whose classic examples are the deuteron of nuclear physics and the helium dimer ${ }^{4} \mathrm{He}_{2}$ molecule. More details about such exotic two-particle states as well as their extensions to few-body systems are given in a recent review (Jensen et al., 2004).

Section III discusses those concepts of diatomic scattering and molecular physics that are particular to the applications of Feshbach resonances in cold gases. Subsection III.A introduces the microscopic origin of the resonance enhancement of interactions illustrated in Fig. 1, scattering channels and rigorous coupled channels theory (Mies et al., 1996; Stoof et al., 1988), as well as the relation between zero energy resonances and molecular energy spectra. Several aspects of the rigorous method are well recovered in terms of two-channel approaches whose general concepts, such as the two-channel Hamiltonian and the meta-stable Feshbach resonance state, are discussed in Subsection III.B. On this basis, Subsection III.C introduces the bare and dressed bound and continuum energy levels. The universal properties of Feshbach molecules near resonance are strictly derived in Subsection III.D Their physical significance is illustrated in Subsection III.E for several experimentally relevant examples, such as molecular binding energies and the lifetimes of dimers in cold Fermi and Bose gases. The size of the magnetic field range associated with universality implies a distinction between entrance- and closed-channel dominated zero energy resonances, whose physical origin is discussed in Subsection III.F Implementations of two-channel approaches are given in detail in Subsection III.G describing properties of Feshbach molecules close to as well as away from zero energy resonances. Their applications involve the Stern-Gerlach separation of dimers shown in Fig. 3. Characteristic parameters relevant to two-channel approaches, such as, for instance, the magnetic moments associated with Feshbach resonances of several atomic species, are summarised in Tables IV]and V.

Section IV reviews dynamical approaches describing the production and dissociation of cold Feshbach molecules via linear magnetic field sweeps across zero energy resonances. Its introductory paragraphs outline, on the basis of the discrete energy spectrum of a trapped atom pair, the adiabatic transfer from quasi-continuum to dimer states. Molecular association of an atom pair via linear magnetic field sweeps falls into the category of rare dynamical two-body problems whose solutions can be represented in an analytic form (Demkov and Osherov, 1968; Macek and Cavagnero, 1998). A detailed derivation of the associated transition amplitudes in Subsection IV.A provides the foundation for all subsequent applications of the Landau-Zener approach (Landau, 1932; Zener, 1932). These involve predictions of final populations of the quasi-continuum to dimer state transfer in tight atom traps, as well as fast sweep limits of molecule production in cold Bose and two spin component Fermi gases. Such conversion efficiencies can be sensitive to the quantum statistics associated with identical atoms whose effects are discussed in Subsection IV.B. The opposite saturation limits of molecule production via magnetic field sweeps in cold gases are a subject of ongoing research requiring dynamical descriptions of many-particle systems. Among such methods, the two-level mean field approach to Bose-Einstein condensates (Timmermans et al., 1999b) is outlined in detail, including its relation to the associated Landau-Zener theory (Góral et al., 2004; Mies et al., 2000). Intuitive as well as quantitative methods in the context of molecular Bose-Einstein condensation illustrated in Fig. 4 are reviewed in Subsection IV.C. outlining the concept of an adiabatic production of dimers via magnetic field sweeps in cold gases with a significant momentum spread. SubsectionIV.D discusses the theory of Feshbach molecular dissociation, demonstrating the accuracy of analytic treatments of linear magnetic field sweeps across zero energy resonances.

Section $\nabla$ addresses the production of dimers via nonlinear magnetic field variations, illustrated, for instance, in Fig. 2] in the context of Ramsey interferometry with atoms and Feshbach molecules. The description of these experiments requires general theoretical concepts, such as a precise treatment of molecular populations in gases, which are obscured in many applications involving linear magnetic field sweeps. Subsection V.A gives an intuitive explanation for the observations of the Ramsey fringes of Fig. 2] using a simplifying two-body approach. The general observable describing the number of molecules in the environment of a gas (Köhler et al., 2003a) is introduced in Subsection V.B. This introduction includes simple applications to the fast sweep limits of dimer production in Bose and two spin component Fermi gases. Subsection V.C addresses the complete description of the Ramsey interferometry experiments in terms of dynamical beyond mean field approaches (Holland et al., 2001; Köhler and Burnett, 2002).

Section VI concludes this review and provides an outlook on related research, such as $p$-wave and optical Feshbach resonances, cold dipolar and ground state molecules as well as extensions to few-body physics and studies of Efimov's effect.

\section{WEAKLY BOUND DIATOMIC MOLECULES}

Weakly bound diatomic molecules are special cases of low energy halo systems (Jensen et al., 2004). These remarkable quantum states are characterised by a large mean separation between the constituent particles which very much exceeds the outer classical turning point, $r_{\text {classical }}$, determined by their binding energy and their attractive interaction. Halos are therefore quite distinct from spatially extended quasi classical states, such as the Rydberg levels associated with the long range Coulomb potential. Due to their non-classical but comparatively simple nature, two-body halos have played a significant role in the present understanding of composite few-particle systems. Classic examples are the deuteron in nuclear physics (Blatt and Weisskopf, 1952) as well as 
the weakly bound helium dimer ${ }^{4} \mathrm{He}_{2}$ molecule (Luo et al. 1993; Schöllkopf and Toennies, 1994), whose typical halo wave function is illustrated in Fig. 5. We note that the modulus of the bound state energy of this halo molecule with respect to the dissociation threshold, i.e. the zero of energy in Fig. 5, is negligibly small compared to the well depth of the pair interaction. The ${ }^{4} \mathrm{He}$ atoms are separated by distances larger than the classical turning point, $r_{\text {classical }}$, with a probability of about $80 \%$. Such distances very much exceed the intuitive force range associated with the potential well.

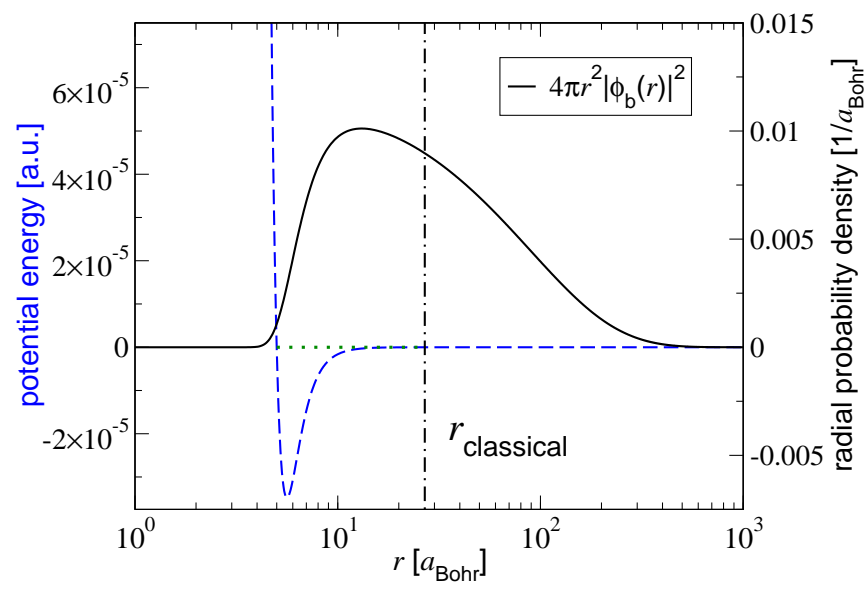

FIG. 5 (Colour in online edition) Helium dimer interaction potential (dashed curve) (Tang et al., 1995) and radial probability density of the ${ }^{4} \mathrm{He}_{2}$ molecule (solid curve). The dotted and dot-dashed lines indicate the bound state energy $E_{\mathrm{b}}=-4.2 \times 10^{-9}$ a.u. ( 1 a.u. $=$ $4.35974417 \times 10^{-18} \mathrm{~J}$ is the atomic unit of energy) and the classical turning point $r_{\text {classical }}=27 a_{\text {Bohr }}\left(a_{\text {Bohr }}=0.052917 \mathrm{~nm}\right.$ is the Bohr radius), respectively. The inter-atomic distance $r$ is given on a logarithmic scale.

The characteristic long range of isotropic diatomic halo molecules, such as the helium dimer, is determined mainly by a single parameter of the interaction potential, the $s$-wave scattering length $a$. Whenever the inter-atomic interaction supports a weakly bound halo state the scattering length is positive. In accordance with effective range theory (Bethe, 1949; Schwinger, 1947a b), the length scale $a$ may be interpreted in terms of the radius of a hypothetical hard sphere which mimics the scattering properties of the real microscopic potential in the limit of zero collision energy. This radius is closely related to the bound state energy of a weakly bound diatomic halo molecule which is well approximated by the formula:

$$
E_{\mathrm{b}}=-\hbar^{2} /\left(m a^{2}\right) \text {. }
$$

Here $m$ is twice the reduced mass of the atom pair which in the case of identical particles coincides with the mass of a single atom. At inter-atomic distances, $r$, large compared to the classical turning point, $r_{\text {classical }}$, the associated isotropic bound state wave function assumes the following general form:

$$
\phi_{\mathrm{b}}(r)=\frac{1}{\sqrt{2 \pi a}} \frac{e^{-r / a}}{r} .
$$

This wave function gives the mean distance between the atoms, i.e. the bond length of the molecule, to be:

$$
\langle r\rangle=4 \pi \int_{0}^{\infty} r^{2} d r r\left|\phi_{\mathrm{b}}(r)\right|^{2}=a / 2 .
$$

For the typical example of the helium dimer in Fig. 5 the solution of the stationary two-body Schrödinger equation with a realistic pair interaction (Tang et al., 1995) predicts $\langle r\rangle=$ $5.1 \mathrm{~nm}$, while the estimate of Eq. (3) yields $\langle r\rangle=5.2 \mathrm{~nm}$. For comparison, measurements based on the diffraction of a helium molecular beam from a micro-fabricated material transmission grating (Grisenti et al., 2000) determined the bond length of ${ }^{4} \mathrm{He}_{2}$ to be $\langle r\rangle=5.2(4) \mathrm{nm}$.

A dependence of physical quantities, such as those of Eqs. (1) and (2), only on the scattering length rather than the details of the microscopic forces is usually referred to as universality. The relation between Eqs. (1) and (2) follows immediately from the stationary Schrödinger equation, $H_{2 \mathrm{~B}} \phi_{\mathrm{b}}(r)=E_{\mathrm{b}} \phi_{\mathrm{b}}(r)$, using the general two-body Hamiltonian associated with the relative motion of an atom pair,

$$
H_{2 \mathrm{~B}}=-\frac{\hbar^{2}}{m} \nabla^{2}+V(r) .
$$

A typical realistic molecular potential $V(r)$ (Tang et al., 1995) is depicted in Fig. 5 for the example of the helium dimer. Its behaviour in the limit of large inter-atomic distances is dominated by the van der Waals interaction,

$$
V(r) \underset{r \rightarrow \infty}{\sim}-C_{6} / r^{6} .
$$

The constant $C_{6}$ is known as the van der Waals dispersion coefficient. In accordance with Eq. (5), the outer classical turning point of the relative motion of an atom pair with the energy $E_{\mathrm{b}}$ of Eq. (1) is given by the following formula:

$$
r_{\text {classical }}=\left[a\left(2 l_{\mathrm{vdW}}\right)^{2}\right]^{1 / 3} .
$$

Here $l_{\mathrm{vdW}}$ is a characteristic range associated with the van der Waals interaction between atoms, usually referred to as the van der Waals length,

$$
l_{\mathrm{vdW}}=\frac{1}{2}\left(m C_{6} / \hbar^{2}\right)^{1 / 4} .
$$

The characteristic property of diatomic halo molecules that their spatial extent, determined by the scattering length $a$, very much exceeds $r_{\text {classical }}$ therefore implies the condition $a \gg l_{\mathrm{vdW}}$ for their existence. In the range of such typically large inter-atomic distances, $r \gg l_{\mathrm{vdW}}$, the stationary Schrödinger equation for a halo molecule reduces to its interaction-free counterpart, i.e.

$$
E_{\mathrm{b}} \phi_{\mathrm{b}}(r) \underset{r \rightarrow \infty}{\sim}-\left(\hbar^{2} / m\right) \nabla^{2} \phi_{\mathrm{b}}(r),
$$

whose unit normalised solution associated with the bound state energy of Eq. (1) is given by Eq. (2).

The universal properties of Eqs. (1) and (2) and the length scales, $a$ and $l_{\mathrm{vdW}}$, associated with diatomic halo systems characterise, in the same manner, the helium dimer as well as 
highly excited long range Feshbach molecules. Any other details of their binary interactions are obscured by the large spatial extent of the bound states. The size of the scattering length, however, which determines the long range of these states depends sensitively on the microscopic potential whose detailed structure varies considerably among different species.

\section{FESHBACH RESONANCES}

Inter-atomic collisions in cold gases are characterised by de Broglie wavelengths much larger than the van der Waals length of the microscopic potential. Similarly to the universal properties of diatomic halo molecules, such a separation of length scales implies that the associated low energy interactions are determined mainly by the $s$-wave scattering length, $a$. Feshbach resonances provide an opportunity of manipulating these inter-atomic forces by exposing a cold gas of alkali atoms to a spatially homogeneous magnetic field of strength $B$. Based on an introduction to the microscopic physics, this section describes the concept of magnetic tuning of the scattering length and the associated cross section (given, for instance, by $8 \pi a^{2}$ in the case of identical Bose atoms) as well as its relation to the properties of molecular states.

\section{A. Molecular physics of resonances}

Resonance enhancement of collision cross sections relies upon the existence of meta-stable states. The two-body molecular physics of such neutral atom Feshbach resonance states is the subject of this subsection. This involves the microscopic origin of the inter-atomic interactions as well as the atomic and molecular symmetries that permit the classification of scattering channels and molecular bound and metastable energy levels. Such a basic understanding of low energy neutral atom scattering and bound states provides the grounding for practical two-channel approaches of the subsequent subsections.

\section{Inter-atomic interactions}

The helium atom of Section $\Pi$ has a ${ }^{1} \mathrm{~S}_{0}$ configuration with no unpaired electron, and the interaction of two such atoms is represented by the single molecular Born-Oppenheimer potential illustrated in Fig.5] Such a system is too simple to have a magnetically tunable resonance state. It is therefore necessary to consider here the experimentally relevant case of a pair of ${ }^{2} S_{1 / 2}$ atoms of the same species. The unpaired electron spins $\mathbf{s}_{1}$ and $\mathbf{s}_{2}$ from each atom can be coupled to a total spin $\mathbf{S}=\mathbf{s}_{1}+\mathbf{s}_{2}$ with the associated quantum numbers $S=0$ or 1 . States with $S=0$ or $S=1$ are called singlet or triplet states, respectively. The electronic part of the inter-atomic interaction is represented, as for the simplest molecule $\mathrm{H}_{2}$ (Pauling, 1939), by singlet and triplet molecular Born-Oppenheimer potentials of ${ }^{1} \Sigma_{g}^{+}$and ${ }^{3} \Sigma_{u}^{+}$symmetry, corresponding respectively to $2 S+1=1$ and 3 . Here the notation " $\Sigma$ " refers to zero projection of the electronic orbital angular momentum on the inter-atomic axis. The label "+" indicates that the electronic wave function is left unchanged upon reflection in a plane containing the nuclei. Finally, " $g$ " and " $u$ " are associated with the gerade (even) and ungerade (odd) symmetry upon inversion through the geometric centre of the molecule, respectively. Consequently, the latter symmetry is absent when the atoms are of different species.

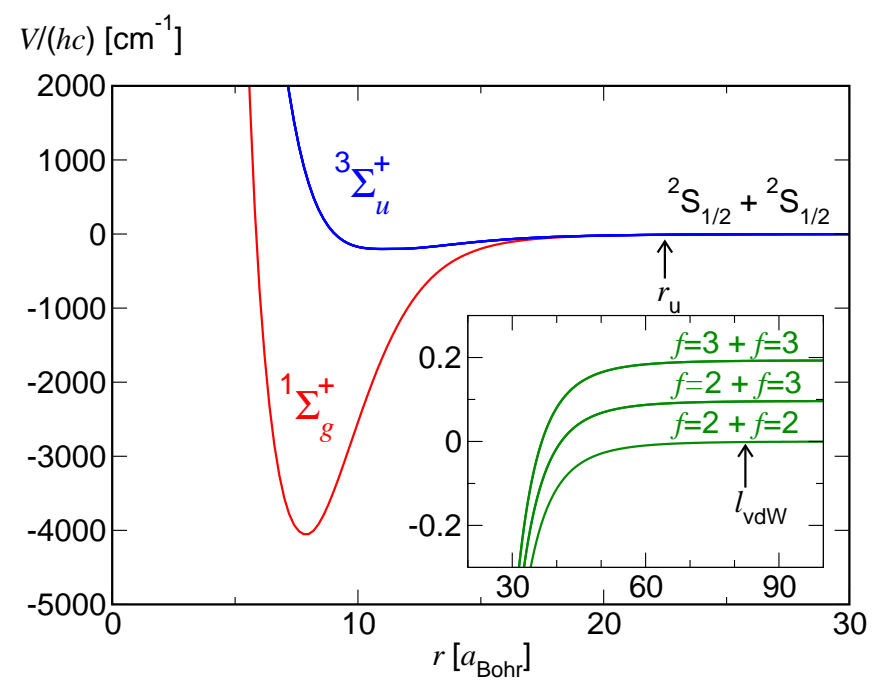

FIG. 6 (Colour in online edition) The ${ }^{1} \Sigma_{g}^{+}$and ${ }^{3} \Sigma_{u}^{+}$BornOppenheimer potential energy curves for the $\mathrm{Rb}_{2}$ dimer molecule correlating with two separated ${ }^{2} \mathrm{~S}_{1 / 2} \mathrm{Rb}$ atoms. An energy $E$ associated with the wave number unit $E / h c=1 \mathrm{~cm}^{-1}$ corresponds to $E / h=29.979 \mathrm{GHz}$. The inset shows the long range adiabatic potentials (Mies et al., 1996, 2000) on a much smaller energy scale with the zero field $(B=0)$ hyperfine structure of the ${ }^{85} \mathrm{Rb}$ isotope with $E_{\mathrm{hf}} / h=3.035 \mathrm{GHz}$. The arrows show the van der Waals length $l_{\mathrm{vdW}}$ of Eq. (7) and the uncoupling distance, $r_{\mathrm{u}}$, where the hyperfine energy $E_{\mathrm{hf}}$ equals the difference between the ${ }^{3} \Sigma_{u}^{+}$and ${ }^{1} \Sigma_{g}^{+}$Born-Oppenheimer potential curves. The ${ }^{87} \mathrm{Rb}$ isotope has the same Born-Oppenheimer potential energy curves, but the long range curves would be different with atomic levels $f=1$ and 2 and $E_{\mathrm{hf}} / h=6.835 \mathrm{GHz}$.

Figure 6 shows potential energy curves $V_{S}(r)$ for $S=0$ and 1 for two $\mathrm{Rb}$ atoms as an illustrative case. Similar potentials exist for any pair of like alkali metal atoms, and if the gerade and ungerade symmetry labels $g$ and $u$ are dropped, for pairs of unlike alkali metal atoms. For the case of two $\mathrm{S}$-state atoms, the long range form of the potential is the van der Waals dispersion with the lead term $-C_{6} / r^{6}$ of Eq. (5) that is identical for the ${ }^{1} \Sigma_{g}^{+}$and ${ }^{3} \Sigma_{u}^{+}$potentials. The splitting between these two potentials comes from the difference in chemical bonding interactions when the charge clouds of the two atoms overlap at small inter-atomic distances, $r<1 \mathrm{~nm}$. The long-range form of this splitting is associated with the electron exchange interaction, which decreases exponentially as $r$ increases. This interaction is attractive for the ${ }^{1} \Sigma_{g}^{+}$state and repulsive for the ${ }^{3} \Sigma_{u}^{+}$state.

More diatomic states need to be accounted for when the atomic nuclear spin quantum number $i$ is nonzero. At zero magnetic field, i.e. $B=0$, the unpaired electrons in each atom with total electronic angular momentum $\mathbf{j}$ can interact with the 


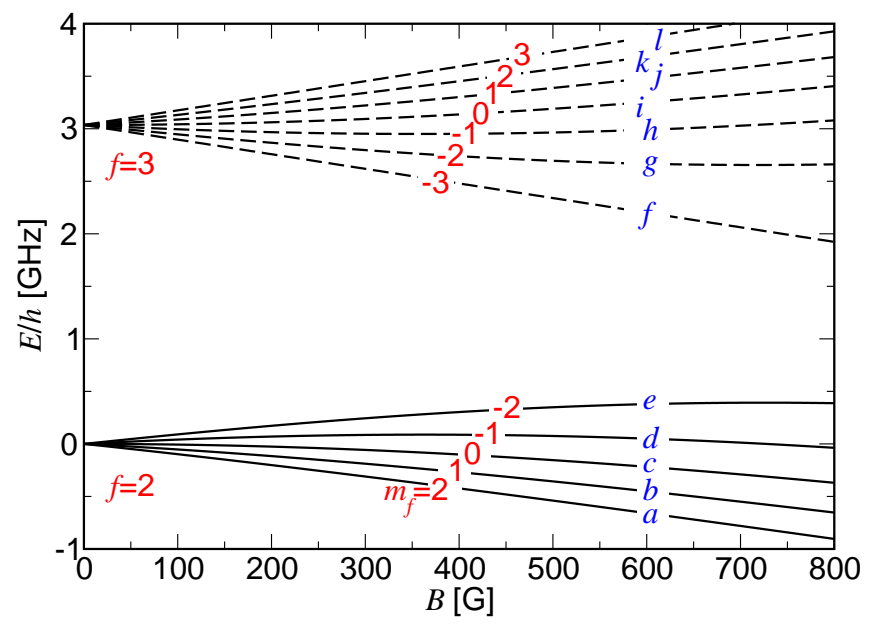

FIG. 7 (Colour in online edition) Zeeman levels of the ${ }^{85} \mathrm{Rb}{ }^{2} \mathrm{~S}_{1 / 2}$ atom versus the magnetic field strength, $B$. Solid and dashed curves refer to the $f=2$ and $f=3$ multiplets, respectively. The projection quantum numbers $m_{f}$ and the alphabetic labels $a, b, c, \ldots$ are shown.

nuclear spin $\mathbf{i}$ to produce the hyperfine levels of total angular momentum $\mathbf{f}=\mathbf{j}+\mathbf{i}$. For alkali metal atoms in their electronic ground states the identity $\mathbf{j}=\mathbf{s}$ gives the total angular momentum quantum number to be either $f=i-1 / 2$ or $i+1 / 2$. The difference in energy between these two hyperfine levels is the hyperfine splitting $E_{\mathrm{hf}}$. If $f$ is an integer, the atom will be a composite boson, whereas if $f$ is half an odd integer, the atom will be a composite fermion. The interaction with a magnetic field will further split the energy of each $f$ state into its Zeeman components. Figure 7 illustrates the $B$-dependent energy levels of ${ }^{85} \mathrm{Rb}$, a bosonic isotope. In the limit of high magnetic field strengths the Zeeman energy of the unpaired electron becomes large compared to $E_{\mathrm{hf}}$. Consequently, $f$ will no longer be a good quantum number. Rotation about the magnetic field axis, however, is a symmetry transformation. This implies that $m_{f}$, the quantum number associated with the projection of the total angular momentum onto this axis, is conserved. Figure 7 also shows that the Zeeman levels of a ${ }^{85} \mathrm{Rb}$ atom do not anti-cross in a range of magnetic fields up to a few hundred G. As for notation, it is therefore often convenient to label the Zeeman levels by $f m_{f}$ where $f$ is the total angular momentum quantum number at $B=0$ with which the level adiabatically correlates. We will use such an explicit reference to the different Zeeman states in the subsequent subsections. An alternative, brief notation (Mies et al., 2000; Stoof et al., 1988) indicated in Fig. 7 is to label the Zeeman components in order of increasing energy as $a, b, c, \ldots$ and shall be used throughout this subsection.

\section{Coupled channels scattering}

The hyperfine splitting $E_{\mathrm{hf}}$ and the magnetic Zeeman energy are typically large compared to the kinetic energy for cold collisions. Consequently, the scattering properties of two atoms depend strongly on the Zeeman levels in which they are prepared. A scattering channel is defined by specifying the quantum numbers that describe each of the two initially separated atoms. In the following it is convenient to perform a partial wave expansion of the stationary scattering wave function of the relative motion of an atom pair. Its components thus depend on the inter-atomic distance, $r$, in addition to the quantum numbers $\ell$ and $m_{\ell}$ associated with the angular momentum of the relative motion and its orientation with respect to the magnetic field axis. Given this convention, a scattering channel is specified by the channel in$\operatorname{dex} \alpha=\left\{f_{1} m_{f_{1}} f_{2} m_{f_{2}} \ell m_{\ell}\right\}$. Here the braces signify that the wave function is (anti-)symmetric with respect to exchange of identical Bose (Fermi) particles (Stoof et al., 1988). Consequently, the order of atomic indices 1 and 2 is unimportant. Only those channels with even (odd) $\ell$ exist for two identical bosons (fermions) in the same Zeeman level. All $\ell$ values are possible for identical bosons or fermions in different Zeeman levels or for nonidentical species. The partial waves associated with $\ell=0,1$, or 2 are designated $s, p$, or $d$ waves. If the channel energy, $E_{\alpha}=E_{f_{1} m_{f_{1}}}+E_{f_{2} m_{f_{2}}}$, is less than the total energy of the system, $E$, the channel $\alpha$ is said to be open. Conversely, if $E_{\alpha}>E$, channel $\alpha$ is referred to as closed. When $E$ is lower than the lowest channel energy, all channels are closed and $E$ can refer only to one of the discrete molecular levels. If $E$ is higher than the lowest channel energy, then at least one channel is open, and $E$ is associated with a stationary energy level in the scattering continuum.

In the case of the ${ }^{1} \mathrm{~S}_{0}{ }^{4} \mathrm{He}$ atom of Section $\prod$ the two-body interaction Hamiltonian is specified by a single-channel potential describing the helium dimer bound state as well as cold collisions. Such a treatment is not adequate for alkali atoms with nuclear spin because of intrinsic coupling among the various channels. It is therefore convenient to represent the wave functions for scattering or bound states as a coupled channels expansion into their components $\Psi_{\alpha}(r, E)$ in the separated atom spin basis labelled by $\alpha$ (Gao, 1996; Mies et al. 1996; Stoof et al., 1988). The associated radial wave function $F_{\alpha}(r, E)$ of the relative motion of an atom pair with the energy $E$ is determined by the following formula:

$$
\Psi_{\alpha}(r, E)=F_{\alpha}(r, E) / r .
$$

Substituting Eq. (9) into the stationary Schrödinger equation gives the following coupled channels equations which, in combination with the boundary conditions imposed on $F_{\alpha}(r, E)$, determine both the continuum and bound states:

$$
\frac{\partial^{2} F_{\alpha}(r, E)}{\partial r^{2}}+\frac{m}{\hbar^{2}} \sum_{\beta}\left[E \delta_{\alpha \beta}-V_{\alpha \beta}(r)\right] F_{\beta}(r, E)=0 .
$$

We note that $m$ should be identified in Eq. (10) with twice the reduced mass of an atom pair. The potential matrix, $V(r)$, takes on the following form in the separated atom spin basis:

$$
V_{\alpha \beta}(r)=\left[E_{f_{1} m_{f_{1}}}+E_{f_{2} m_{f_{2}}}+\frac{\hbar^{2} \ell(\ell+1)}{m r^{2}}\right] \delta_{\alpha \beta}+V_{\alpha \beta}^{\mathrm{int}}(r) .
$$

Here the atomic hyperfine and magnetic interaction terms are given by the experimentally known separated atom energies 
$E_{f_{1} m_{f_{1}}}$ and $E_{f_{2} m_{f_{2}}}$, and the kinetic energy of axis rotation is given by the centrifugal energy term proportional to $\ell(\ell+1)$. These terms are diagonal in the asymptotic basis. The complicated part of the scattering due to the electronic BornOppenheimer potentials and electron spin-dependent interactions is contained in the interaction matrix $V_{\text {int }}(r)$ of Eq. (11), which is comprised of two parts:

$$
V_{\mathrm{int}}(r)=V_{\mathrm{el}}(r)+V_{\mathrm{ss}}(r) .
$$

The contribution $V_{\mathrm{el}}(r)$ represents the strong electronic interaction. It is diagonal in $\ell$ but non-diagonal in the atomic channel quantum numbers $f_{1} m_{f_{1}} f_{2} m_{f_{2}}$. Its elements diagonal in $f_{1} m_{f_{1}} f_{2} m_{f_{2}}$ depend on weighted sums of the two BornOppenheimer potentials $V_{S=0}(r)$ and $V_{S=1}(r)$, whereas the offdiagonal terms depend on the difference between $V_{S=0}(r)$ and $V_{S=1}(r)$. The strong electronic interaction, $V_{\mathrm{el}}(r)$, is responsible for elastic scattering and inelastic spin-exchange collisions (Bender, 1963; Dalgarno and Rudge, 1965), and gives rise to the broadest scattering resonances.

The term $V_{\mathrm{ss}}(r)$ in Eq. (12) represents the weak relativistic spin-spin potential energy (Moerdijk et al., 1995; Stoof et al., 1988). It is due to the anisotropic dipolar interaction between the two electron spins, and is non-diagonal in both $f_{1} m_{f_{1}} f_{2} m_{f_{2}}$ and $\ell$, i.e., it couples different partial waves. In the limit of large inter-atomic distances $V_{\mathrm{ss}}(r)$ is proportional to $\alpha^{2} / r^{3}$, where $\alpha=1 / 137.0426$ is the fine structure constant. As $V_{\mathrm{ss}}(r)$ is a tensor operator of rank 2, only blocks that differ in $\ell$ by zero or two units have non-vanishing matrix elements, according to the Wigner-Eckart theorem (Eckart, 1930; Wigner, 1927). In addition, there are no $s$-wave diagonal potentials varying as $1 / r^{3}$.

At short range the spin-dipole interaction can be modified by second-order spin-orbit contributions, which are important for a heavy atom like Cs (Kotochigova et al., 2001; Mies et al., 1996). In general, the potential energy contribution $V_{\mathrm{ss}}(r)$ is responsible for weak inelastic spin-dipolar relaxation and gives rise to narrow scattering resonances.

The low energy collision physics of alkali atoms in specific hyperfine states is sensitive to the re-coupling of electron spins between the separated atoms and the short range zone of strong chemical interactions. At small inter-atomic distances the potential energy scale is orders of magnitude larger than $E_{\mathrm{hf}}$. Whereas the electron spin is coupled to the nuclear spin on the same atom when the atoms are far separated, the electron spins become uncoupled from the nuclear spin and couple strongly to one another at small $r$ to make the $S=0$ and 1 states of the Born-Oppenheimer potentials. The distance where this re-coupling occurs is near $r_{\mathrm{u}}$ of Fig. 6 where the difference in Born-Oppenheimer potentials $V_{S=1}\left(r_{\mathrm{u}}\right)-V_{S=0}\left(r_{\mathrm{u}}\right)$ due to the exchange potential is equal to the atomic hyperfine energy $E_{\mathrm{hf}}$. This occurs typically in the distance range of 20 to $25 a_{\text {Bohr }}$ for alkali atoms.

The coupled channels method (Mies et al., 1996; Stoof et al., 1988) of Eq. (10) properly accounts for the dynamical changes in the couplings among the five angular momenta $\mathbf{s}_{1}, \mathbf{s}_{2}, \mathbf{i}_{1}, \mathbf{i}_{2}$, and $\boldsymbol{\ell}$ as the atoms move through the region near $r_{\mathrm{u}}$. Basic symmetries of the coupling terms in $V_{\text {int }}(r)$ of Eq. (12) allow us to separate the interaction matrix into blocks, within which the coupling is strong and between which the coupling is intrinsically weak. Such a separation gives rise to classifications of the various stationary energy states in terms of their predominant symmetry properties. Projected levels that are bound just within a particular block are, in general, associated with scattering resonances when their energy is above the scattering threshold. We note, however, that in contrast to the complete stationary states determined by Eq. (10), such Feshbach resonance levels depend on the separation of $V_{\text {int }}(r)$ into blocks or, equivalently, on the choice of basis set.

In this context, the separated atom spin basis referred to in Eq. (11) is convenient at long range, but leads to off-diagonal elements at short range. One alternative, short range basis would first couple $\mathbf{s}_{1}$ and $\mathbf{s}_{2}$ to a resultant $\mathbf{S}$ and $\mathbf{i}_{1}$ and $\mathbf{i}_{2}$ to a resultant $\mathbf{I}$. Then $\mathbf{S}$ and $\mathbf{I}$ can be coupled to a resultant $\mathbf{F}$, which in turn couples to $\ell$ to give the total angular momentum $\mathbf{F}_{\text {total }}$ Moerdijk et al., 1995; Tiesinga et al., 1993). Then we could set up a molecular basis set with quantum numbers $(S I) F \ell F_{\text {total }} M$. Here $M$ refers to the orientation quantum number associated with the projection of $\mathbf{F}_{\text {total }}$ onto the magnetic field axis. This short range basis takes advantage of the fact that $V_{\mathrm{el}}(r)$ is diagonal in $S$. An alternative separated atom basis set could couple $\mathbf{f}_{1}$ and $\mathbf{f}_{2}$ to a resultant $\mathbf{F}$, and give the basis $\left(f_{1} f_{2}\right) F \ell F_{\text {total }} M$. This basis is useful at low $B$ fields at which the Zeeman levels do not anti-cross, and where $F$ may be viewed as a good quantum number. The unitary transformation between separated atom and molecular basis sets is called a frame transformation (Bender, 1963; Burke et al., 1998; Dalgarno and Rudge, 1965; Gao et al., 2005). Bound and meta-stable states of light elements like $\mathrm{Li}$ and $\mathrm{Na}$ are best classified by the molecular basis (Moerdijk et al., 1995; Simonucci et al., 2005) whereas the separated atom basis is better for heavy elements like $\mathrm{Rb}$ or Cs (Chin et al., 2004b; Marte et al., 2002). Similarly, the long-range part of a scattering wave function is best described in the separated atom basis set, whereas the molecular basis is more appropriate for the short range part.

\section{Threshold collisions}

The quantum numbers $f_{1} m_{f_{1}}$ and $f_{2} m_{f_{2}}$ associated with the Zeeman states of the separated atoms in which a dilute gas is prepared determine the entrance channel of a two-body collision. In the context of cold collisions, it is usually sufficient to consider just the $s$-wave $(\ell=0)$ component of an initial plane wave momentum state of the relative motion of an atom pair. To avoid inelastic processes known as spin relaxation, most experimental applications of Feshbach resonances involve atom pairs in the lowest energetic Zeeman states for which $s$-wave scattering is allowed. In the following, we assume such a case and choose the zero of energy at the entrance channel energy, $E_{\alpha}$. This convention implies that the total energy $E$ of a colliding atom pair is identical to its positive kinetic energy, $\hbar^{2} k^{2} / m$. Here $k$ is referred to as the wave number and $p=\hbar k$ is the momentum of the relative motion. On the other hand, bound state energies, $E_{\mathrm{b}}$, are always negative. 
The amplitudes associated with transitions between the initial and final states of a diatomic collision may be inferred from the asymptotic form of the scattering solutions to Eq. (10) in the limit of large distances, $r \rightarrow \infty$. As the only open channel is assumed to be the entrance channel, the long range boundary condition imposed on the associated component of the radial wave function reads (Taylor, 1972):

$$
F_{\alpha}\left(r, \hbar^{2} k^{2} / m\right) \propto \sin [k r+\xi(\hbar k)] .
$$

Here the absolute magnitude of $F_{\alpha}\left(r, \hbar^{2} k^{2} / m\right)$ is determined up to an overall energy dependent pre-factor whose value is a matter of convention. All information about a collision is contained in the scattering phase shift, $\xi(\hbar k)$. According to effective range theory (Bethe, 1949; Schwinger, 1947a b), $\xi(\hbar k)$ determines the scattering length, $a$, via the following low energy asymptotic expansion:

$$
k \cot \xi(\hbar k)=-\frac{1}{a}+\frac{1}{2} k^{2} r_{\mathrm{eff}} .
$$

Here $r_{\text {eff }}$ is known as the effective range. For most of the applications in this review, the scattering length alone is sufficient for the description of cold diatomic collisions.

In general, the scattering length is only weakly dependent on the magnetic field strength, $B$, unless $B$ can be tuned in such a way that a closed channel Feshbach resonance level crosses the entrance channel scattering threshold. Such a scenario may occur due to a difference in magnetic moments, $\partial E_{\alpha} / \partial B$ and $\partial E_{\beta} / \partial B$, associated with the entrance and closed channels, respectively. The meta-stability of the resonance state leads to a time delay during a collision when the energies of the scattered atoms and of the resonance level are nearly matched. This results in an enhancement of the scattering cross section whose width in energy depends on the strength of the coupling between the entrance and closed channels via the lifetime of the resonance state. As the zero energy cross section is proportional to $a^{2}$, such a resonance enhancement of collisions may be used to widely tune the scattering length (Tiesinga et al., 1993) as illustrated in Fig. 1 We note, however, that because of the inter-channel coupling the magnetic field strength $B_{0}$ associated with the singularity of $a$ differs from the crossing point between the resonance energy and the scattering threshold. The magnetic field width and shift of such zero energy resonances will be the subject of Subsection III.C.

Singularities of the $s$-wave scattering length are always accompanied by the degeneracy of a bound vibrational level with the scattering threshold (Taylor, 1972). In the context of magnetic Feshbach resonances, the properties of such a coupled channels stationary energy state, termed the Feshbach molecule, may be inferred from Eq. (10) in the zero bound state energy limit, $E_{\mathrm{b}} \rightarrow 0$. Similarly to the studies of Section II the derivation just relies upon the fact that the potential matrix $V_{\text {int }}(r)$ of Eq. (11) vanishes at large separations, $r \rightarrow \infty$. In such an asymptotic distance range the atoms cease to interact, and the solution of Eq. (10) associated with any $s$-wave $(\ell=0)$ channel is given by the following formula:

$$
F_{\alpha}\left(r, E_{\mathrm{b}}\right) \propto \exp \left[-\sqrt{-m\left(E_{\mathrm{b}}-E_{\alpha}\right)} r / \hbar\right] .
$$

Its pre-factor depends on the overall normalisation of the bound state and is thus determined by all components. In the limit $E_{\mathrm{b}} \rightarrow 0$, however, only the entrance channel radial wave function acquires a long range and, therefore, predominates all the others. The relation between the binding energy and the scattering length in the vicinity of a zero energy resonance will be the subject of Subsection III.D This discussion will show, on the basis of general arguments, that $E_{\mathrm{b}}$ is determined by Eq. (1), while the Feshbach molecular wave function reduces to its entrance channel component given by Eq. (2).

If the entrance channel is not the lowest in energy the above scenario of zero energy resonances and Feshbach molecules is only approximate. This implies that the scattering length is always finite even when a closed channel Feshbach resonance level is magnetically tuned to cross the entrance channel energy. The associated Feshbach molecule can decay into the lower energetic open channels via spin relaxation (Köhler et al., 2005; Thompson et al., 2005b). Such a rather general situation underlies, for instance, the experiments of Fig. 2 involving gases of ${ }^{85} \mathrm{Rb}$, and serves as an example for the following explicit channel classification.

\section{Example of channel classification}

The most rigorous classification of scattering channels is by the total projection quantum number $M=m_{f_{1}}+m_{f_{2}}+m_{\ell}$. In the absence of external fields other than $B$, the symmetry with respect to rotation about the magnetic field axis implies that $M$ is strictly conserved during the course of a collision, i.e., states with different $M$ can not couple. The next useful classification is by the partial wave quantum number $\ell$. Only weak coupling is normally possible between states of different $\ell$, since it can only originate from the intrinsically small and anisotropic $V_{\mathrm{ss}}(r)$ matrix elements.

TABLE I Separated atom quantum numbers for the $s$-wave $(\ell=0)$ $M=-4$ block of the coupling matrix $V_{\mathrm{el}}(r)$ for ${ }^{85} \mathrm{Rb}$, for which $f$

\begin{tabular}{|c|c|c|c|c|}
\hline$\left(f_{1} f_{2}\right)$ & $m_{f_{1}} m_{f_{2}}$ & $\alpha$ & $\begin{array}{c}E_{\alpha} / h[\mathrm{GHz}] \\
\text { for } B=0\end{array}$ & $\begin{array}{c}E_{\alpha} / h[\mathrm{GHz}] \\
\text { for } B=160 \mathrm{G}\end{array}$ \\
\hline (2) & $-2-2$ & $e e$ & 0 & 0 \\
\hline (2 3) & $-1-3$ & $d f$ & 3.035732 & 2.591623 \\
\hline (2 3) & $-2-2$ & $e g$ & 3.035732 & 2.756754 \\
\hline (3 3 3) & $-3-1$ & $f h$ & 6.071464 & 5.508558 \\
\hline (3 3 ) & $-2-2$ & $g g$ & 6.071464 & 5.513508 \\
\hline
\end{tabular}
assumes the values 2 and 3 . The separated atom energies relative to $E_{e e}=0$ are shown for $B=0$ and $160 \mathrm{G}(16.0 \mathrm{mT})$.

Table 1 shows an example of the quantum numbers needed to describe the $s$-wave channels associated with the interaction of a pair of $f=2, m_{f}=-2{ }^{85} \mathrm{Rb}$ atoms, which is the $e$ state in the alphabetic notation. This state is one for which there is a broad Feshbach resonance close to the scattering threshold near $155 \mathrm{G}$. Both the binding energies of the associated Feshbach molecules and the scattering length may be inferred from solutions of Eq. (10), using just the matrix elements of the potential $V_{\mathrm{el}}(r)$ between the channel states of 
Table 【 Since the nuclear spin of a ${ }^{85} \mathrm{Rb}$ atom is $i=5 / 2$, the two ground state $f$ values are 2 and 3 . The total projection quantum number for $s$ waves is $M=-4$ for any $B$. There are only five possible separated atom spin channels. As the hyperfine splitting is $E_{\mathrm{hf}} / h=3.035 \mathrm{Ghz}$ for this species, cold collisions associated with temperatures $T$ on the order of $1 \mu \mathrm{K}$ (where $k_{\mathrm{B}} T / h=21 \mathrm{kHz}$ given the Boltzmann constant $k_{\mathrm{B}}=1.3806505 \times 10^{-23} \mathrm{~J} / \mathrm{K}$ ) have only a single open $s$-wave channel, the ee channel. All the other $\ell=0$ channels, $d f, e g$, $f h$, and $g g$ are closed.

TABLE II Separated atom quantum numbers for the open channel $d$-wave $(\ell=2) M=-4$ block of the coupling matrix $V_{\mathrm{ss}}(r)$ for ${ }^{85} \mathrm{Rb}$. The separated atom energies relative to $E_{e e}=0$ are shown for $B=$ 0 and $160 \mathrm{G}(16.0 \mathrm{mT})$. There are also 19 closed channels in the $d$ wave $M=-4$ block.

\begin{tabular}{|c|c|c|c|c|}
\hline$\left(f_{1} f_{2}\right)$ & $m_{f_{1}} m_{f_{2}} m_{\ell}$ & $\alpha$ & $\begin{array}{c}E_{\alpha} / h[\mathrm{GHz}] \\
\text { for } B=0\end{array}$ & $\begin{array}{c}E_{\alpha} / h[\mathrm{GHz}] \\
\text { for } B=160 \mathrm{G}\end{array}$ \\
\hline (2) & $-1-1-2$ & $d d$ & 0 & -0.161496 \\
\hline (2) & $0-2-2$ & ce & 0 & -0.157321 \\
\hline (2 2) & $-1-2-1$ & de & 0 & -0.080748 \\
\hline (2) & $\begin{array}{lll}-2 & -2 & 0\end{array}$ & $e e$ & 0 & 0 \\
\hline
\end{tabular}

The anisotropic interaction $V_{\mathrm{ss}}(r)$ weakly couples the $s$ wave block to the $d$-wave block, and the $d$-wave block to the $g$-wave block, etc. This has two consequences: extra spinrelaxation channels are possible, and projected energy states of $d$-wave character (or even higher partial wave character) can give rise to scattering resonances for $s$-wave collisions. In the ${ }^{85} \mathrm{Rb}$ case there are a total of $23 M=-4 d$-wave channels that couple to the $s$-wave block illustrated in Table [. Only the four listed in Table [I] are open with respect to the ee channel energy. These channels are all degenerate with the ee $s$-wave channel at $B=0$. Such a degeneracy at zero magnetic field implies a suppression of inelastic collisions due to the Wigner threshold law (Wigner, 1948) outlined in Subsection II.D. The $d$-wave channels of Table II become open, however, by a relatively large amount of energy on the cold $\mu \mathrm{K}$ temperature scale as $B$ increases. The associated energy gap gives rise to inelastic decay by which a pair of $e$-state atoms can relax in a collision when $B$ increases from zero (Roberts et al., 2000b). These open $d$-wave channels are also responsible for the observed spontaneous dissociation of Feshbach molecules with binding energies near the $e e$ scattering threshold (Thompson et al., 2005b). Associated lifetimes as a function of the magnetic field strength will be discussed in Subsection II.E

Table III illustrates the blocks of the matrix $V_{\text {int }}(r)$ according to $\left\{f_{1} f_{2}\right\} F \ell$ quantum numbers. The example refers to Bose atoms with a nuclear spin quantum number of $i=5 / 2$, such as ${ }^{85} \mathrm{Rb}$. Basis states in the same vertical column of Table III that is, with the same $F \ell$ quantum numbers, are coupled by the strong exchange interactions in $V_{\mathrm{el}}(r)$. Basis states from different vertical columns can only be coupled by the weak interactions in $V_{\mathrm{ss}}(r)$. Classification using $F \ell$ blocks was used for Feshbach resonance states with $\ell>0$ observed for the comparatively heavy alkali metal atoms ${ }^{87} \mathrm{Rb}$ (Marte et al.,
TABLE III Separated atom quantum numbers $\left(f_{1} f_{2}\right) F \ell$ for the $s$ and $d$-wave part of $V_{\text {int }}(r)$ for the bosonic isotope ${ }^{85} \mathrm{Rb}$, for which $f$ assumes the values 2 and 3 . Odd values of $F$ are missing when $f_{1}$ equals $f_{2}$, because of Bose symmetry. Spin-exchange interactions couple states in the same column, whereas states in different columns can only be coupled by spin-dipolar interactions.

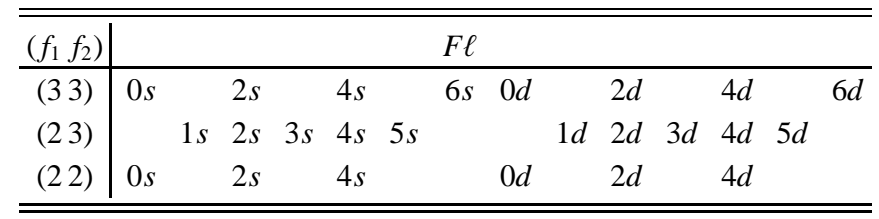

2002) and ${ }^{133} \mathrm{Cs}$ (Chin et al., 2004b). In particular, the ${ }^{133} \mathrm{Cs}_{2}$ Feshbach molecules associated with the Stern-Gerlach separation experiments of Fig. 3 are mainly of $g$-wave character with $F=4$ and $\ell=4$. Such a separated atom classification shall be applied in the following to Feshbach resonance and molecular states of ${ }^{85} \mathrm{Rb}$ associated with the observations of Fig. 2]

\section{Near threshold bound states}

The illustration of the relation between resonance and bound states on the one hand and singularities of the scattering length on the other hand relies upon coupled channels calculations. Figure 8 shows the five separated atom channel energies $E_{\alpha}(B)$ for the $s$-wave block described in Table 【In accordance with the convention used throughout this review, the scattering threshold associated with the ee entrance channel defines the zero of energy. As the five channel energies just reflect the sums of their associated atomic Zeeman energies, they cluster into three groups. The lowest energy group consists just of the $e e$ entrance channel. The next group with energies on the order of $E_{\mathrm{hf}}$ includes the $d f$ and $e g$ channels. Finally, the group with energies of about $2 E_{\mathrm{hf}}$ refers to the $f h$ and $g g$ channels. Both channel energies associated with each one of the latter two groups are degenerate at $B=0$. At the low magnetic field strengths shown in Fig. 8, the choice of $\left(f_{1} f_{2}\right) F$ quantum numbers well characterises the two-body physics. The (22) entrance channel has $F=4$, the (23) group has $F=4$ and 5 and the (33) group has $F=4$ and 6 . Only $F$ values of 4 or more are possible because the projection quantum number is $M=-4$; the odd value $F=5$ is ruled out for identical bosons with $\left(f_{1} f_{2}\right)=(33)$.

The solid curves in Figure 8 show the $s$-wave bound states of the ${ }^{85} \mathrm{Rb}_{2}$ molecular dimer with negative energies, $E_{\mathrm{b}}<0$. The curves virtually parallel to the $E=0$ threshold refer to the $v=-1,-2$, and -3 states of the $e e$ entrance channel, labelled by their vibrational quantum numbers, $v$, starting with $v=-1$ for the highest excited level. In addition to $v$, their low field (22)4 quantum numbers are indicated in Fig. 8. These levels all have nearly the same magnetic moment as a pair of separated atoms in the entrance channel Zeeman state configuration. Each one of the other four, closed channels also has a vibrational series leading to each of the four closed-channel thresholds. The pair of (23) $F, v=-3$ levels with $F=4$ and 


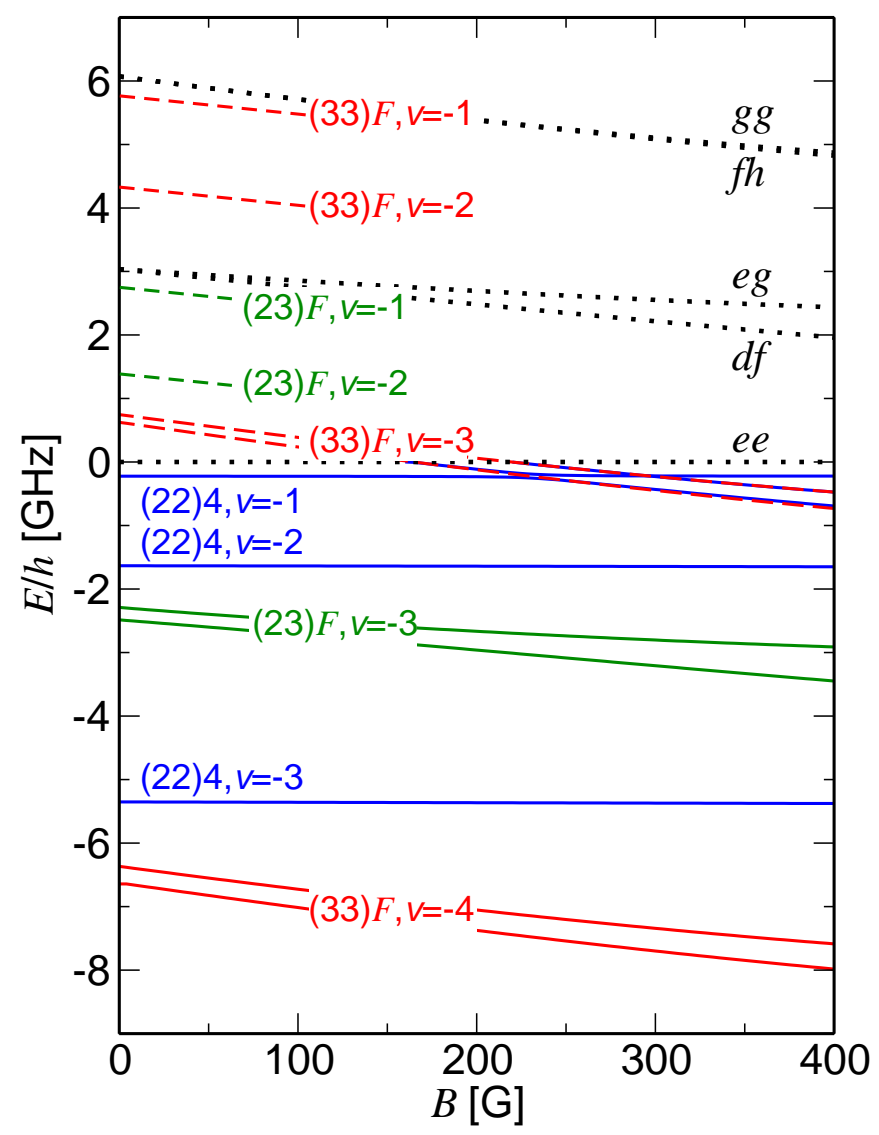

FIG. 8 (Colour in online edition) Magnetic field dependent $M=-4$ $s$-wave energy levels of the ${ }^{85} \mathrm{Rb}_{2}$ dimer. The dotted curves labelled by $e e, d f, e g, f h$ and $g g$ show the energies of the five separated atom channels of Table $\square$ Solid curves indicate the calculated $s$-wave coupled channels bound state energies labelled by the vibrational quantum number, $v$. Their symmetry labels refer to the set of quantum numbers $\left(f_{1} f_{2}\right) F$. The $e e$ limit gives rise to a single vibrational progression with $F=4$; the $d f$ and $e g$ limits give rise to two (23) $F$ series with $F=4$ and 5; and the $f h$ and $g g$ limits give rise to two (33) $F$ series with $F=4$ and 6 . In all cases, the $F=4$ level has the lower energy of the $\left(f_{1} f_{2}\right)$ pairs. At $B=0$ the $v=-1$ and $-2,(23) F$ levels and the $v=-1,-2$, and $-3,(33) F$ levels represent meta-stable states with $E>0$ embedded in the (22) 4 ee scattering continuum. These meta-stable levels are represented by dashed curves, which give the approximate positions of scattering resonances in the $e e$ channel. The meta-stable levels with $F=4$ are coupled to the $e e s$-wave scattering continuum through the exchange interaction. The $F=5$ and 6 metastable levels are not exchange coupled to the $e e$ entrance channel at $B=0$, but become weakly coupled at higher fields where $F$ is no longer a good quantum number.

5 and the pair of (33) $F, v=-4$ levels with $F=4$ and 6 are both bound with respect to the separated atoms. For both pairs the lowest level has $F=4$. These levels have different magnetic moments from a pair of $e e$ separated atoms. This implies that their bound state energies, $E_{\mathrm{b}}(B)$, relative to $E=0$ vary significantly with the magnetic field strength $B$.

While the ${ }^{85} \mathrm{Rb}_{2}$ molecular dimer states involve the complete strong electronic interaction, $V_{\mathrm{el}}(r)$, the Feshbach resonance levels of Fig. 8 refer to blocks of the potential matrix

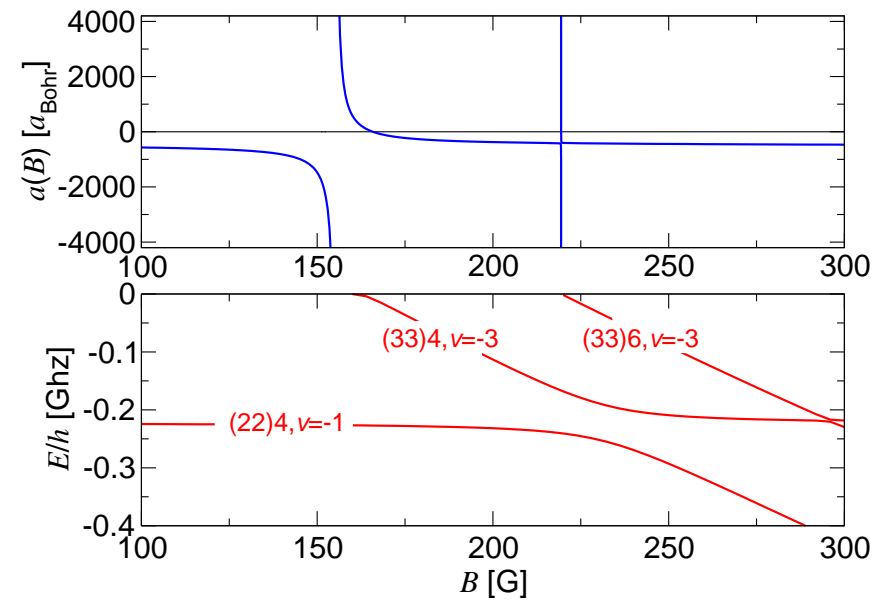

FIG. 9 (Colour in online edition) Scattering length (upper panel) and bound state energy levels (lower panel) versus $B$ for two ${ }^{85} \mathrm{Rb}$ atoms in the $e$ state. The separated atom energy of two $e$-state atoms is taken to be zero. A singularity of the scattering length occurs where an energy level becomes degenerate with the scattering threshold at $E=0$. The resolution of the figure is not sufficient to show the variation of the bound state energies with $B$ just below threshold; this variation is discussed in Fig. [12 of Subsection III.D The broad resonance is due to the threshold crossing of the strongly coupled $\left(f_{1} f_{2}\right) F \ell=(33) 4 s, v=-3$ level, whereas the narrow resonance is due to the crossing of the weakly coupled $\left(f_{1} f_{2}\right) F \ell=(33) 6 s, v=-3$ level.

associated with their symmetry labels. At zero magnetic field the (23), $v=-1$ and -2 levels and the (33), $v=-1,-2$, and -3 levels have positive energies so that they are meta-stable levels embedded in the ee scattering continuum. The two (33) $F$ levels are especially interesting, in that they cross the ee $E=0$ threshold and turn into bound states when $B$ is sufficiently large. Figure 9 shows an expanded view of the bound state energy levels in the near-threshold crossing region as well as the $s$-wave scattering length $a$ of the $e e$ entrance channel as a function of $B$. The scattering length has a singularity at the magnetic field value $B_{0}$ where a new bound state appears with $E_{\mathrm{b}}\left(B_{0}\right)=0$. The $F=4$ (33) level is coupled by the exchange interaction to the $F=4 e e$ entrance channel, and gives rise to a broad resonance with an associated singularity of $a$ near $155 \mathrm{G}$. On the other hand, the $F=6$ (33) level is only weakly coupled to the $F=4 e e$ entrance channel, and gives rise to a narrow zero energy resonance near $220 \mathrm{G}$. In addition, the interaction between the (33) $F$ levels and the highest excited entrance channel bound state at $E_{-1} / h=-0.22 \mathrm{GHz}$ is evident in Fig. 9. It results, for instance, in the avoided crossing of the (22) $4, v=-1$ and (33) $4, v=-3$ levels between $200 \mathrm{G}$ and $250 \mathrm{G}$ which is due to the same coupling that leads to the broad singularity of the scattering length near $155 \mathrm{G}$.

\section{B. Two-channel approach}

Coupled channels calculations based on realistic molecular potentials and known atomic properties are capable of accounting for a variety of experimental colli- 
sional and bound state properties of alkali-metal species van Abeelen and Verhaar, 1998; Bartenstein et al., 2005; Chin et al., 2004b; Houbiers et al., 1998; Leo et al., 2000; Loftus et al., 2002; Marcelis et al., 2004; Marte et al., 2002). Their accuracy, including predictions of new resonances, is possible once the actual potentials have been calibrated in such a way that they recover the correct scattering lengths for the Born-Oppenheimer ${ }^{1} \Sigma_{o}^{+}$and ${ }^{3} \Sigma_{u}^{+}$potentials (Abraham et al., 1997; van Kempen et al., 2002) in addition to the van der Waals coefficient, $C_{6}$. Coupled channels approaches have the drawback, however, that they are not readily accessible. Consequently, it is desirable to find simpler approaches to Feshbach resonance and molecular levels close to the dissociation threshold energy. To this end, the physics of binary collisions as well as the properties of the highly excited molecular bound states can usually be well described in terms of just two scattering channels (Child, 1974; Mies et al., 2000; Moerdijk et al., 1995; Timmermans et al., 1999b). In the following, we denote by the entrance channel the Zeeman state configuration of a pair of asymptotically separated atoms in which a dilute gas is initially prepared. Under the conditions of resonance enhancement, this spin configuration is strongly coupled, in general, to several energetically closed scattering channels. In idealised treatments, however, this coupling is usually due to the near degeneracy of the energies of a single meta-stable vibrational state, the Feshbach resonance state $\phi_{\text {res }}(r)$, and the colliding atoms. The spin configuration associated with the Feshbach resonance state is referred to, in the following, simply as the closed channel.

\section{Two-channel Hamiltonian}

The general Hamiltonian of the relative motion of an atom pair, i.e., the basis of all two-channel approaches (Child, 1974; Mies et al., 2000; Moerdijk et al., 1995; Timmermans et al., 1999 b), is given by the following matrix:

$$
H_{2 \mathrm{~B}}=\left(\begin{array}{cc}
H_{\mathrm{bg}} & W(r) \\
W(r) & H_{\mathrm{cl}}(B)
\end{array}\right) .
$$

Its off diagonal elements, $W(r)$, are the energies associated with the spin exchange (or dipole) interaction and provide the inter-channel coupling as a function of the distance $r$ between the atoms. The diagonal elements, $H_{\mathrm{bg}}$ and $H_{\mathrm{cl}}(B)$, can be interpreted in terms of entrance and closed channel Hamiltonians in the hypothetical absence of coupling, respectively. These single channel Hamiltonians consist of kinetic and potential energy contributions given by the following formulae:

$$
\begin{aligned}
H_{\mathrm{bg}} & =-\frac{\hbar^{2}}{m} \nabla^{2}+V_{\mathrm{bg}}(r), \\
H_{\mathrm{cl}}(B) & =-\frac{\hbar^{2}}{m} \nabla^{2}+V_{\mathrm{cl}}(B, r) .
\end{aligned}
$$

Here and in the following we choose the zero of energy as the threshold for dissociation of the entrance channel. Consequently, the background scattering potential $V_{\mathrm{bg}}(r)$ of Eq. (17) vanishes in the limit of large inter-atomic separations, in accordance with the general asymptotic behaviour of the van der Waals interaction described by Eq. (5). In this approach the complete two-body Hamiltonian of Eq. (16) depends on the magnetic field strength $B$ simply via an overall shift of the closed channel potential $V_{\mathrm{cl}}(B, r)$ with respect to the entrance channel dissociation threshold. The amount of this shift is determined by the sum of the single particle Zeeman energies associated with the closed channel spin configuration of a pair of atoms. Typical diagonal potential energy contributions to the two-body Hamiltonian are illustrated schematically in Fig. 10, whereas special requirements for them will be discussed in Subsection III.G.

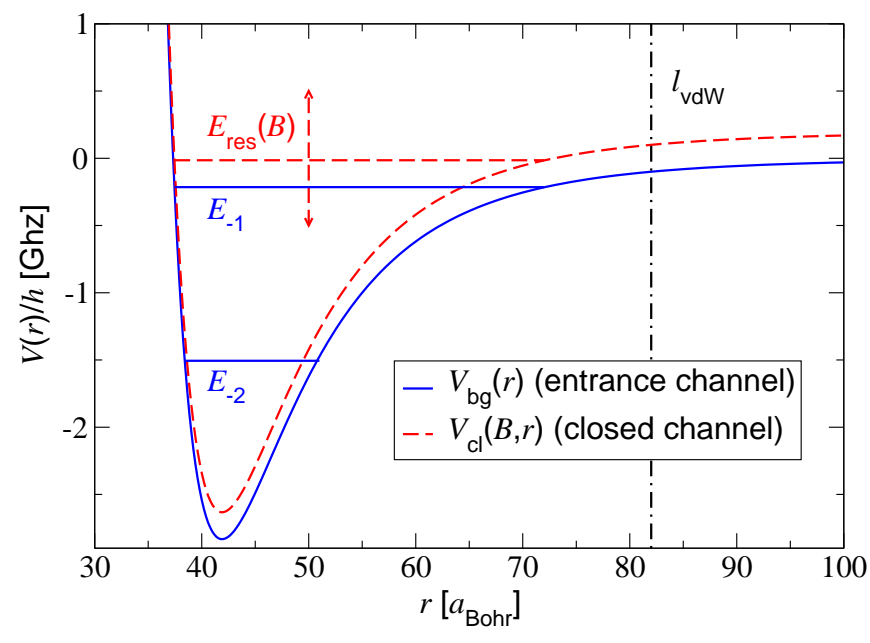

FIG. 10 (Colour in online edition) Scheme of the entrance (solid curve) and closed channel (dashed curve) potentials associated with a model representation of the $155 \mathrm{G}$ zero energy resonance of ${ }^{85} \mathrm{Rb}$ (Köhler et al., 2003b). The horizontal dashed and vertical dot-dashed lines indicate the magnetic field dependent energy $E_{\mathrm{res}}(B)$ of the Feshbach resonance level $\phi_{\text {res }}(r)$ and the van der Waals length of $l_{\mathrm{vdW}}=82 a_{\mathrm{Bohr}}$, respectively. In this model the outer classical turning point of the resonance level $\phi_{\text {res }}(r)$ associated with the energy $E_{\text {res }}(B)$ in the closed channel potential is located at about $r_{\text {classical }}=72 a_{\text {Bohr }}$. Its vibrational quantum number $v=-1$ with respect to the dissociation threshold of $V_{\mathrm{cl}}(B, r)$ is chosen arbitrarily. The horizontal solid lines indicate the energies $E_{-1}$ and $E_{-2}$ of the bare vibrational bound states associated with the entrance channel potential. This bare interaction is adjusted in such way that it mimics the highest excited $\left(f_{1} f_{2}\right) F=(22) 4, v=-1$ and -2 levels of Fig. 8 In this model the off-diagonal spin exchange coupling has been chosen to be $W(r)=\beta \exp (-r / \alpha)$, using $\alpha=5 a_{\mathrm{Bohr}}$ and $\beta / k_{\mathrm{B}}=38.5 \mathrm{mK}$ to match the measured Feshbach resonance parameters (Claussen et al., 2003).

\section{The bare Feshbach resonance state}

The closed channel Hamiltonian supports the bare Feshbach resonance state in accordance with the following Schrödinger equation:

$$
H_{\mathrm{cl}}(B) \phi_{\mathrm{res}}(r)=E_{\mathrm{res}}(B) \phi_{\mathrm{res}}(r) .
$$


The associated resonance energy, $E_{\text {res }}(B)$, can be tuned with respect to the entrance channel dissociation threshold, i.e. the zero of energy in Fig. 10, by varying the magnetic field strength. In the hypothetical absence of inter-channel coupling the Feshbach resonance level usually represents a comparatively tightly bound state of $H_{\mathrm{cl}}(B)$ with a classical radius $r_{\text {classical }}$ smaller than the van der Waals length of Eq. (7). Under realistic conditions, however, $\phi_{\text {res }}(r)$ is only a metastable state with a decay width depending on the strength of the inter-channel coupling. Its precise form can not always be unambiguously identified from full coupled channels calculations because the two-channel picture is an idealisation. The remarkable accuracy of such a simplifying approach is due to the fact that near resonance the physically relevant dressed stationary energy levels are largely insensitive to the detailed structure of the resonance state.

\section{Dressed energy states}

The dressed stationary energy states consist of two orthogonal components associated with the spin configurations $|\mathrm{bg}\rangle$ and $|c l\rangle$ of the entrance and closed channels, respectively. Their wave functions are therefore of the following general form (Drummond et al., 1998): $|\mathrm{bg}\rangle \phi_{\mathrm{bg}}(\mathbf{r})+|\mathrm{cl}\rangle \phi_{\mathrm{cl}}(\mathbf{r})$. The components $\phi_{\mathrm{bg}}(\mathbf{r})$ and $\phi_{\mathrm{cl}}(\mathbf{r})$ depend on the relative position $\mathbf{r}$ of the atoms. In accordance with Eq. 16, these components are determined by the following set of coupled stationary Schrödinger equations:

$$
\begin{aligned}
H_{\mathrm{bg}} \phi_{\mathrm{bg}}(\mathbf{r})+W(r) \phi_{\mathrm{cl}}(\mathbf{r}) & =E \phi_{\mathrm{bg}}(\mathbf{r}), \\
W(r) \phi_{\mathrm{bg}}(\mathbf{r})+H_{\mathrm{cl}}(B) \phi_{\mathrm{cl}}(\mathbf{r}) & =E \phi_{\mathrm{cl}}(\mathbf{r}) .
\end{aligned}
$$

All applications in this review will involve either symmetric or anti-symmetric spin configurations of identical Bose and Fermi atom pairs, respectively. Their physical spatial wave functions will only consist of an $s$-wave component. The solutions of Eqs. (20) and (21) with a negative energy $E$ below the entrance channel dissociation threshold are associated with molecular bound states. Solutions with a positive energy belong to the continuum spectrum of the Hamiltonian and describe collisions of initially separated pairs of atoms.

\section{Dressed continuum states}

The dressed continuum states can be labelled by the relative momentum $\mathbf{p}$ of a pair of asymptotically separated atoms in the entrance channel spin configuration and are associated with the collision energies, $E=p^{2} / m$. Due to the continuous range of angles of incidence between the atoms, any given collision energy is infinitely degenerate. In this context it is convenient to postpone the introduction of any symmetry properties of the spatial wave functions associated with a possible identical nature of the atoms. Continuum states are usually chosen in such a way that their entrance channel component, $\phi_{\mathbf{p}}^{\mathrm{bg}}(\mathbf{r})$, behaves at asymptotically large inter-atomic distances like a superposition of an incident plane wave and an outgoing spherical wave (Taylor, 1972). This choice of the wave function leads to the following boundary condition:

$$
\phi_{\mathbf{p}}^{\mathrm{bg}}(\mathbf{r}) \underset{r \rightarrow \infty}{\sim} \frac{1}{(2 \pi \hbar)^{3 / 2}}\left[e^{i \mathbf{p} \cdot \mathbf{r} / \hbar}+f(\vartheta, p) \frac{e^{i p r / \hbar}}{r}\right] .
$$

The quantity $f(\vartheta, p)$ is known as the scattering amplitude and depends on the modulus $p$ of the relative momentum of the atoms and on the scattering angle determined by the relation: $\cos \vartheta=\mathbf{p} \cdot \mathbf{r} /(p r)$. Due to the off diagonal coupling, $W(r)$, the stationary continuum states also have a closed channel component. The associated wave function, $\phi_{\mathbf{p}}^{\mathrm{cl}}(\mathbf{r})$, decays at asymptotically large inter-atomic distances because the cold collision energies of interest are below the closed channel dissociation threshold. This property reflects physical intuition as closed channel atom pairs are spatially confined by the potential $V_{\mathrm{cl}}(r)$ of Fig. 10 .

Due to their long range of Eq. (22), stationary continuum wave functions may be interpreted in terms of amplitudes for the density of particle flux rather than physical states (Taylor, 1972). In the present context, the entrance channel component, $\phi_{\mathbf{p}}^{\mathrm{bg}}(\mathbf{r})$, determines observable low energy scattering properties of colliding atom pairs, such as, for instance, the differential cross section of distinguishable atoms, $|f(\vartheta, p)|^{2}$. These properties can be represented in terms of bare energy states associated with the Hamiltonians $H_{\mathrm{bg}}$ and $H_{\mathrm{cl}}(B)$. To this end, it is instructive to reformulate the coupled set of stationary Schrödinger equations (20) and (21), including the boundary condition of Eq. (22), in terms of the associated Green's functions in addition to the entrance channel continuum states. The bare Green's functions depend on a complex variable $z$ with the dimension of an energy in accordance with the following formulae:

$$
\begin{aligned}
G_{\mathrm{bg}}(z) & =\left(z-H_{\mathrm{bg}}\right)^{-1}, \\
G_{\mathrm{cl}}(B, z) & =\left[z-H_{\mathrm{cl}}(B)\right]^{-1} .
\end{aligned}
$$

The entrance channel continuum wave functions, $\phi_{\mathbf{p}}^{(+)}(\mathbf{r})$, also referred to as background scattering states, satisfy the stationary Schrödinger equation associated with the Hamiltonian $H_{\text {bg }}$ of Eq. (17), i.e.:

$$
H_{\mathrm{bg}} \phi_{\mathbf{p}}^{(+)}(\mathbf{r})=\frac{p^{2}}{m} \phi_{\mathbf{p}}^{(+)}(\mathbf{r}) .
$$

Their long range behaviour is determined by boundary conditions analogous to Eq. (22) with $f(\vartheta, p)$ replaced by the bare amplitude, $f_{\mathrm{bg}}(\vartheta, p)$, associated with the background scattering.

Expressed in terms of the bare Green's functions and continuum states, the Schrödinger equations 20) and 21) read:

$$
\begin{aligned}
\left|\phi_{\mathbf{p}}^{\mathrm{bg}}\right\rangle & =\left|\phi_{\mathbf{p}}^{(+)}\right\rangle+G_{\mathrm{bg}}(E+i 0) W\left|\phi_{\mathbf{p}}^{\mathrm{cl}}\right\rangle \\
\left|\phi_{\mathbf{p}}^{\mathrm{cl}}\right\rangle & =G_{\mathrm{cl}}(B, E) W\left|\phi_{\mathbf{p}}^{\mathrm{bg}}\right\rangle
\end{aligned}
$$

The argument " $z=E+i 0$ " of the entrance channel Green's function in Eq. (26) indicates that the physical collision energy $E=p^{2} / m$ is approached from the upper half of the complex plane. This choice of the energy argument ensures 
that the scattering wave function $\phi_{\mathbf{p}}^{\mathrm{bg}}(\mathbf{r})$ is compatible with Eq. (22), in accordance with the following long range asymptotic behaviour of $G_{\mathrm{bg}}(z)$ in spatial coordinates:

$$
G_{\mathrm{bg}}\left(z, \mathbf{r}, \mathbf{r}^{\prime}\right) \underset{r \rightarrow \infty}{\sim}-\frac{m(2 \pi \hbar)^{3 / 2}}{4 \pi \hbar^{2}} \frac{e^{i p r / \hbar}}{r}\left[\phi_{\mathbf{p}}^{(-)}\left(\mathbf{r}^{\prime}\right)\right]^{*} .
$$

Here $\phi_{\mathbf{p}}^{(-)}\left(\mathbf{r}^{\prime}\right)=\left[\phi_{-\mathbf{p}}^{(+)}\left(\mathbf{r}^{\prime}\right)\right]^{*}$ is the entrance channel continuum energy state with incoming spherical wave boundary conditions (Taylor, 1972). Its label, $\mathbf{p}=(m E)^{1 / 2} \mathbf{r} / r$, may be interpreted as the asymptotic momentum associated with the relative motion of the scattered atoms. The closed channel continuum wave function $\phi_{\mathbf{p}}^{\mathrm{cl}}(\mathbf{r})$ of Eq. 27 decays at asymptotically large inter-atomic distances because the bare Green's function on the right hand side is evaluated at the collision energy $E=p^{2} / m$ below the dissociation threshold of $V_{\mathrm{cl}}(r)$.

Direct application of the two-channel Hamiltonian (16) to Eqs. 26) and (27) verifies their equivalence to the coupled Schrödinger equations (20) and (21). The associated set of integral equations for the spatial wave functions $\phi_{\mathbf{p}}^{\mathrm{bg}}(\mathbf{r})$ and $\phi_{\mathbf{p}}^{\mathrm{cl}}(\mathbf{r})$ may be used, for instance, to numerically determine the exact dressed energy states in the two-channel approach (Köhler et al., 2003b). This formulation of the two-channel scattering problem is also particularly useful for an approximate treatment based on the singularities of the closed channel Green's function. The single resonance approach, underlying this treatment, provides analytic formulae for the dressed continuum wave functions as well as their associated collision parameters in terms of the bare energy states.

\section{Single resonance approach}

In accordance with Eqs. (19) and (24), the closed channel Green's function has a singularity at the resonance energy $E_{\mathrm{res}}(B)$. Provided that the Feshbach resonance state $\phi_{\text {res }}(r)$ is unit normalised, i.e. $\left\langle\phi_{\text {res }} \mid \phi_{\text {res }}\right\rangle=1$, the singular diagonal matrix element of $G_{\mathrm{cl}}(B, E)$ is given by the following formula:

$$
\left\langle\phi_{\mathrm{res}}\left|G_{\mathrm{cl}}(B, E)\right| \phi_{\mathrm{res}}\right\rangle=\left[E-E_{\mathrm{res}}(B)\right]^{-1} \text {. }
$$

Based on Eq. 29), the single resonance approach takes advantage of the near degeneracy of the resonance energy with the entrance channel dissociation threshold illustrated in Fig. 10 . At typical cold collision energies, $E=p^{2} / m$, the resonance detuning $\Delta E(B)=E-E_{\text {res }}(B)$ in Eq. (29) is negligible compared to the spacings between the discrete energy levels of $H_{\mathrm{cl}}(B)$. Consequently, the closed channel Green's function in Eq. (27) is dominated by its virtually singular diagonal contribution associated with the Feshbach resonance level. These estimates thus lead to the following approximation (Child, 1974; Góral et al., 2004):

$$
G_{\mathrm{cl}}(B, E) \approx\left|\phi_{\text {res }}\right\rangle \frac{1}{\Delta E(B)}\left\langle\phi_{\text {res }}\right| .
$$

The single resonance approach consists in replacing the bare Green's function in Eq. (27) by the right hand side of Eq. (30). This replacement implies that the functional form of the closed channel component of the dressed continuum wave function $\phi_{\mathbf{p}}^{\mathrm{cl}}(\mathbf{r})$ is given by the Feshbach resonance state $\phi_{\text {res }}(r)$. The associated overlap factor $\left\langle\phi_{\text {res }} \mid \phi_{\mathbf{p}}^{\text {cl }}\right\rangle$ is determined by the energy and magnetic field dependent amplitude:

$$
A(B, E)=\left\langle\phi_{\text {res }}|W| \phi_{\mathbf{p}}^{\mathrm{bg}}\right\rangle / \Delta E(B) .
$$

The dressed state component $\left|\phi_{\mathbf{p}}^{\mathrm{cl}}\right\rangle$ on the right hand side of Eq. (26) can then also be eliminated in favour of the product of $\left|\phi_{\text {res }}\right\rangle$ and $A(B, E)$. These replacements in Eqs. (26) and (27) give the following explicit formulae for the dressed continuum states in terms of the bare Feshbach resonance and background scattering states:

$$
\begin{aligned}
\left|\phi_{\mathbf{p}}^{\mathrm{bg}}\right\rangle & =\left|\phi_{\mathbf{p}}^{(+)}\right\rangle+G_{\mathrm{bg}}(E+i 0) W\left|\phi_{\text {res }}\right\rangle A(B, E), \\
\left|\phi_{\mathbf{p}}^{\mathrm{cl}}\right\rangle & =\left|\phi_{\text {res }}\right\rangle A(B, E) .
\end{aligned}
$$

The amplitude $A(B, E)$ of Eq. (31) may be expressed in terms of the same bare states by inserting Eq. (32) into Eq. (31). This yields:

$$
A(B, E)=\frac{\left\langle\phi_{\text {res }}|W| \phi_{\mathbf{p}}^{(+)}\right\rangle}{\Delta E(B)-\left\langle\phi_{\text {res }}\left|W G_{\mathrm{bg}}(E+i 0) W\right| \phi_{\text {res }}\right\rangle} .
$$

We note that the dressed continuum wave functions of Eqs. (32) and (33) depend on the magnetic field strength only through the detuning $\Delta E(B)$ of the resonance energy $E_{\text {res }}(B)$ in the denominator of Eq. (34). Within the typical range of experimental magnetic field strengths $E_{\text {res }}(B)$ is a linear function of $B$ to a good approximation. This linear dependence may be represented in terms of an expansion of $E_{\text {res }}(B)$ about the resonant field, $B_{\text {res }}$, at which the Feshbach resonance level crosses the dissociation threshold of the entrance channel. In accordance with the choice of the zero of energy in Fig. 10, $B_{\text {res }}$ is determined by the relation $E_{\text {res }}\left(B_{\text {res }}\right)=0$. This implies the simple formula (Moerdiik et al., 1995):

$$
E_{\text {res }}(B)=\mu_{\text {res }}\left(B-B_{\text {res }}\right) .
$$

Here the slope of the linear curve, $\mu_{\text {res }}$, is the difference between the magnetic moments of the Feshbach resonance state and a pair of asymptotically separated non-interacting atoms.

The single resonance approach of Eqs. (32), 33) and (34) gives an exact representation of the dressed continuum states provided that the spatial configuration of a closed channel atom pair is restricted to the Feshbach resonance state $\phi_{\text {res }}(r)$. Such an assumption is associated with the following formal replacement of the closed channel Hamiltonian:

$$
H_{\mathrm{cl}}(B) \rightarrow\left|\phi_{\text {res }}\right\rangle E_{\text {res }}(B)\left\langle\phi_{\text {res }}\right| .
$$

This simplification of the complete two-channel Hamiltonian of Eq. (16) yields low energy scattering amplitudes, $f(\vartheta, p)$, sufficiently accurate to determine the magnetic field dependence of the $s$-wave scattering length $a(B)$.

\section{Width and shift of a zero energy resonance}

The $s$-wave scattering length is determined by the long range asymptotic behaviour associated with the dressed con- 
tinuum states in terms of the zero momentum limit of the scattering amplitude. This limit is well represented by the following partial wave analysis (Taylor, 1972):

$$
f(\vartheta, p)=\sum_{\ell=0}^{\infty}(2 \ell+1) f_{\ell}(p) P_{\ell}(\cos \vartheta) \underset{p \rightarrow 0}{\sim}-a .
$$

Here $\ell$ labels the quantum number associated with the orbital angular momentum and $P_{\ell}(\cos \vartheta)$ is a Legendre polynomial. The $s$-wave scattering amplitude is related to the phase shift of Eq. (13) through the formula $f_{0}(p)=\hbar \exp [i \xi(p)] \sin \xi(p) / p$. We note that the limit $p \rightarrow 0$ implies rotational symmetry of the entire wave function $\phi_{\mathbf{p}}^{\mathrm{bg}}(\mathbf{r})$, i.e. independence of the scattering angle $\vartheta$, because the incident plane wave in Eq. (22) is isotropic at zero momentum. The higher angular momentum components $f_{\ell}(p)$ of the scattering amplitude, besides the $s$ wave associated with $\ell=0$, are usually negligible in applications to binary collisions in cold gases, due to their proportionality to $p^{2 \ell}$.

The behaviour of $\phi_{\mathbf{p}}^{\mathrm{bg}}(\mathbf{r})$ at asymptotically large inter-atomic distances $r$ can be inferred from Eq. (28) and from the bare amplitude, $f_{\mathrm{bg}}(\vartheta, p)$, associated with the background scattering wave function, $\phi_{\mathbf{p}}^{(+)}(\mathbf{r})$. In accordance with the explicit representation of the dressed continuum wave function of Eq. (32), the scattering amplitude is thus given by the following expression:

$$
f(\vartheta, p)=f_{\mathrm{bg}}(\vartheta, p)-\frac{m(2 \pi \hbar)^{3}\left\langle\phi_{\mathbf{p}}^{(-)}|W| \phi_{\mathrm{res}}\right\rangle}{4 \pi \hbar^{2}} A(B, E) .
$$

In the zero momentum limit this expression recovers the resonance enhanced $s$-wave scattering length in terms of the formula (Moerdijk et al., 1995):

$$
a(B)=a_{\mathrm{bg}}\left(1-\frac{\Delta B}{B-B_{0}}\right) .
$$

Its parameters are the background scattering length, $a_{\mathrm{bg}}$, the width, $\Delta B$, and the position of the zero energy resonance, $B_{0}$. The background scattering length is associated with the bare scattering amplitude, $f_{\mathrm{bg}}(\vartheta, p)$, via a relation analogous to Eq. (37). The zero momentum limit of Eq. (38) determines the width of the resonance to be:

$$
\Delta B=\frac{m(2 \pi \hbar)^{3}}{4 \pi \hbar^{2} a_{\mathrm{bg}} \mu_{\mathrm{res}}}\left|\left\langle\phi_{\mathrm{res}}|W| \phi_{0}^{(+)}\right\rangle\right|^{2} .
$$

We note that Eq. (39) predicts the scattering length $a(B)$ to assume all values from $-\infty$ to $+\infty$ due to its singularity at $B_{0}$, the measurable magnetic field strength at which the zero energy resonance occurs. The width, $\Delta B$, characterises the distance in magnetic fields between the position of the singularity, $B_{0}$, and the zero of the scattering length. The denominator of the amplitude $A(B, E=0)$ of Eq. (34) determines the physical resonance position, $B_{0}$, relative to the zero energy crossing point of the bare Feshbach resonance level, $B_{\text {res }}$, to be:

$$
B_{0}=B_{\text {res }}-\left\langle\phi_{\text {res }}\left|W G_{\text {bg }}(0) W\right| \phi_{\text {res }}\right\rangle / \mu_{\text {res }} .
$$

The absolute magnitude of $B_{0}$ enters the two-channel approach as an adjustable parameter because the magnetic field strength $B_{\text {res }}$ is not directly measurable. The resonance shift, $B_{0}-B_{\text {res }}$, however, determines characteristic properties of the highest excited dressed vibrational bound state, such as, e.g., the admixture of the closed channel spin configuration to its wave function.

\section{Dressed molecular bound states}

In analogy to Eqs. (26) and (27), the two-channel dressed molecular bound states are determined in terms of the bare Green's functions by coupled matrix equations of the following form:

$$
\begin{aligned}
\left|\phi_{\mathrm{b}}^{\mathrm{bg}}\right\rangle & =G_{\mathrm{bg}}\left(E_{\mathrm{b}}\right) W\left|\phi_{\mathrm{b}}^{\mathrm{cl}}\right\rangle, \\
\left|\phi_{\mathrm{b}}^{\mathrm{cl}}\right\rangle & =G_{\mathrm{cl}}\left(B, E_{\mathrm{b}}\right) W\left|\phi_{\mathrm{b}}^{\mathrm{bg}}\right\rangle .
\end{aligned}
$$

The discrete bound state energies, $E_{\mathrm{b}}$, are negative, i.e. below the dissociation threshold of the entrance channel. Both channels are therefore closed and, consequently, the atom pairs are confined by the potential wells of $V_{\mathrm{bg}}(r)$ and $V_{\mathrm{cl}}(r)$ in Fig. 10, Their associated wave functions, $\phi_{\mathrm{b}}^{\mathrm{bg}}(\mathbf{r})$ and $\phi_{\mathrm{b}}^{\mathrm{cl}}(\mathbf{r})$, vanish accordingly in the limit of large inter-atomic distances, similarly to the long range asymptotic behaviour of diatomic halo molecules of Eq. (2). The components of the dressed twochannel bound states can therefore be interpreted in terms of probability amplitudes, subject to the normalisation condition:

$$
\left\langle\phi_{\mathrm{b}}^{\mathrm{bg}} \mid \phi_{\mathrm{b}}^{\mathrm{bg}}\right\rangle+\left\langle\phi_{\mathrm{b}}^{\mathrm{cl}} \mid \phi_{\mathrm{b}}^{\mathrm{cl}}\right\rangle=1 .
$$

Figure 11 illustrates the magnetic field dependence of the highest excited dressed vibrational bound state, referred to in the following as the Feshbach molecule, for the example of the $155 \mathrm{G}$ zero energy resonance of ${ }^{85} \mathrm{Rb}$. The wave functions $\phi_{\mathrm{b}}^{\mathrm{bg}}(\mathbf{r})$ and $\phi_{\mathrm{b}}^{\mathrm{cl}}(\mathbf{r})$ represent exact solutions of Eqs. (42) and (43) associated with the model potentials of Fig. 10 using $\mu_{\text {res }} / h=-3.46 \mathrm{MHz} / \mathrm{G}$. The entrance channel components $\phi_{\mathrm{b}}^{\mathrm{bg}}(\mathbf{r})$ of Fig. 11 are extended over a wide range of inter-atomic distances. This range increases beyond all limits as the magnetic field strength $B$ approaches the zero energy resonance position of $B_{0} \approx 155 \mathrm{G}$. The bond length of the Feshbach molecules in Fig. 11, i.e. their mean inter-atomic distance, is given, for instance, by $\langle r\rangle=521 a_{\mathrm{Bohr}}$ at $160 \mathrm{G}$ and $4255 a_{\mathrm{Bohr}}$ at $155.5 \mathrm{G}$. Figure 11 also suggests that the functional form of the closed channel component, $\phi_{\mathrm{b}}^{\mathrm{cl}}(\mathbf{r})$, is virtually independent of $B$. Numerical studies (Köhler et al., $2003 \mathrm{~b})$ reveal that $\phi_{\mathrm{b}}^{\mathrm{cl}}(\mathbf{r})$ is proportional to the bare resonance wave function, $\phi_{\text {res }}(r)$, which indicates the applicability of Eq. (36). The probability of detecting an atom pair in the closed channel spin configuration, i.e. $\left\langle\phi_{\mathrm{b}}^{\mathrm{cl}} \mid \phi_{\mathrm{b}}^{\mathrm{cl}}\right\rangle=$ $\int d \mathbf{r}\left|\phi_{\mathrm{b}}^{\mathrm{cl}}(\mathbf{r})\right|^{2}$, decreases from about $4.7 \%$ at $B=160 \mathrm{G}$ to only $0.1 \%$ at $B=155.5 \mathrm{G}$. Given that $\phi_{\mathrm{b}}^{\mathrm{cl}}(\mathbf{r})$ is spatially confined in the same manner as the resonance state, the suppression of the closed channel component is a consequence of the increasing range of $\phi_{\mathrm{b}}^{\mathrm{bg}}(\mathbf{r})$ and the normalisation condition of 


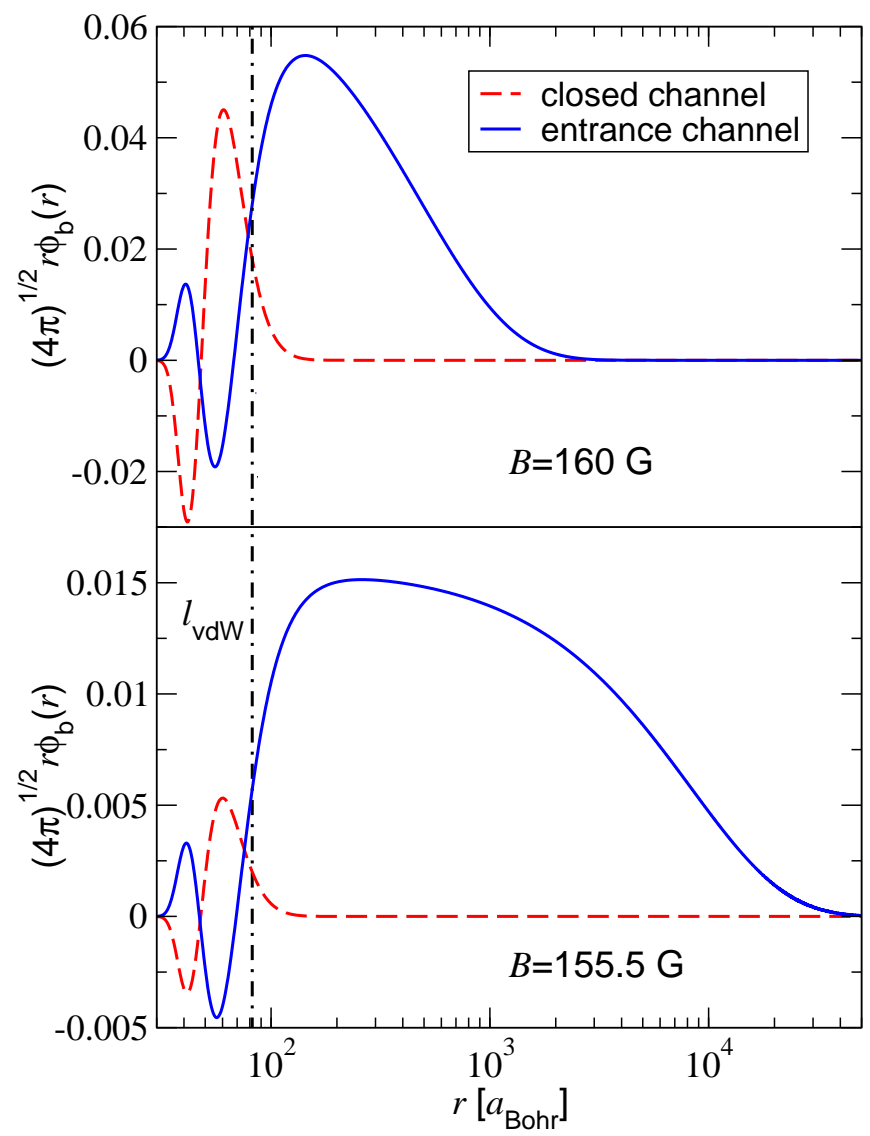

FIG. 11 (Colour in online edition) Entrance and closed channel components of the highest excited vibrational bound state associated with the $155 \mathrm{G}$ zero energy resonance of ${ }^{85} \mathrm{Rb}$ versus the inter-atomic distance $r$ (Köhler et al., 2003b). The wave functions were determined using the model illustrated in Fig. 10 for magnetic field strengths of $160 \mathrm{G}$ (upper panel) and $155.5 \mathrm{G}$ (lower panel), respectively. The nodes of the wave functions at short distances are associated with the vibrational states supported by the bare potentials. The long range behaviour of the entrance channel wave functions, beyond the van der Waals length of $82 a_{\text {Bohr }}$ (dot-dashed line), is largely determined just by the binding energy of the Feshbach molecule (Köhler et al., 2003a). We note that the inter-atomic distance is given on a logarithmic scale.

Eq. (44). All these trends reflect general properties of Feshbach molecules which can be readily explained using the single resonance approach.

In analogy to the treatment of the dressed continuum levels, the single resonance approach to the two-channel bound states is equivalent to the pole approximation to the closed channel Green's function of Eq. (30). This approximation renders Eq. (43) into a practical form. Its analytic solution may be inserted into Eq. (42) which yields the following unit normalised dressed molecular two-component state:

$$
\left(\begin{array}{c}
\left|\phi_{\mathrm{b}}^{\mathrm{bg}}\right\rangle \\
\left|\phi_{\mathrm{b}}^{\mathrm{cl}}\right\rangle
\end{array}\right)=\frac{1}{\mathcal{N}_{\mathrm{b}}}\left(\begin{array}{c}
G_{\mathrm{bg}}\left(E_{\mathrm{b}}\right) W\left|\phi_{\mathrm{res}}\right\rangle \\
\left|\phi_{\mathrm{res}}\right\rangle
\end{array}\right)
$$

Here $\mathcal{N}_{\mathrm{b}}$ is the associated normalisation constant whose ex- plicit expression reads:

$$
\mathcal{N}_{\mathrm{b}}=\sqrt{1+\left\langle\phi_{\text {res }}\left|W G_{\mathrm{bg}}^{2}\left(E_{\mathrm{b}}\right) W\right| \phi_{\text {res }}\right\rangle} .
$$

In the single resonance approach all bound state energies $E_{\mathrm{b}}$ are determined by a constraint on Eq. (45) which can be derived by multiplying Eq. (42) by $\left\langle\phi_{\text {res }}\right| W$ from the left. This leads to the following formula:

$$
E_{\mathrm{b}}=\mu_{\text {res }}\left(B-B_{\text {res }}\right)+\left\langle\phi_{\text {res }}\left|W G_{\mathrm{bg}}\left(E_{\mathrm{b}}\right) W\right| \phi_{\text {res }}\right\rangle .
$$

We note that Eq. (47) recovers Eq. (41) in the limits $E_{\mathrm{b}} \rightarrow 0$ and $B \rightarrow B_{0}$, provided that the magnetic field strength approaches $B_{0}$ from the side of positive scattering lengths. This confirms that the binding energy of the Feshbach molecule vanishes at the measurable resonance position $B_{0}$. Such a weak bond implies that the properties of the near resonant highest excited dressed vibrational state are determined solely by the scattering length, $a$, in analogy to the general findings with respect to halo dimers in Section $\Pi$. The range of magnetic field strengths in which the Feshbach molecular state as well as its energy depend just on $a$ is usually referred to as the universal regime.

\section{Universal properties of Feshbach molecules}

In accordance with the results of Section $\Pi$ the properties of halo dimers, such as, e.g., the large spatial extent of their wave functions in Fig. 11, can all be inferred from Eq. (1), the universal formula for their binding energy.

\section{Universal binding energy}

The formal derivation of Eq. (1) for Feshbach molecules (Góral et al., 2004) relies upon an explicit determination of the matrix element involving $G_{\mathrm{bg}}\left(E_{\mathrm{b}}\right)$ on the right hand side of Eq. (47). This may be performed on the basis of the following resolvent identity:

$$
G_{\mathrm{bg}}\left(E_{\mathrm{b}}\right)=G_{\mathrm{bg}}(0)-E_{\mathrm{b}} G_{\mathrm{bg}}(0) G_{\mathrm{bg}}\left(E_{\mathrm{b}}\right) .
$$

Multiplication of Eq. (48) by $G_{\mathrm{bg}}^{-1}(0)=-H_{\mathrm{bg}}$ from the left and by $G_{\mathrm{bg}}^{-1}\left(E_{\mathrm{b}}\right)=E_{\mathrm{b}}-H_{\mathrm{bg}}$ from the right readily verifies this identity. The contribution to Eq. (47) from the first term, $G_{\mathrm{bg}}(0)$, on the right hand side of Eq. (48) yields the energy shift $\mu_{\text {res }}\left(B_{\text {res }}-B_{0}\right)$ due to Eq. (41). To evaluate the contribution from the second term, $-E_{\mathrm{b}} G_{\mathrm{bg}}(0) G_{\mathrm{bg}}\left(E_{\mathrm{b}}\right)$, it is instructive to employ the spectral decomposition of the bare entrance channel Green's function:

$$
G_{\mathrm{bg}}(z)=\int d \mathbf{p} \frac{\left|\phi_{\mathbf{p}}^{(+)}\right\rangle\left\langle\phi_{\mathbf{p}}^{(+)}\right|}{z-p^{2} / m}+G_{\mathrm{bg}}^{\mathrm{b}}(z) .
$$

Here the energy argument $z$ is either zero or $E_{\mathrm{b}}$. The quantity $G_{\mathrm{bg}}^{\mathrm{b}}(z)$ includes all contributions to $G_{\mathrm{bg}}(z)$ from the bare bound states whose energies, depicted in Fig. 10, are usually far detuned from typical energies of Feshbach molecules 
in the universal regime. Thus neglecting $G_{\mathrm{bg}}^{\mathrm{b}}(z)$ in the product $-E_{\mathrm{b}} G_{\mathrm{bg}}(0) G_{\mathrm{bg}}\left(E_{\mathrm{b}}\right)$ determines the zero bound state energy limit of Eq. (47) to be:

$$
\mu_{\text {res }}\left(B-B_{0}\right)-m^{2} E_{\mathrm{b}} \int d \mathbf{p} \frac{\left|\left\langle\phi_{\text {res }}|W| \phi_{\mathbf{p}}^{(+)}\right\rangle\right|^{2}}{p^{2}\left(p^{2}+m\left|E_{\mathrm{b}}\right|\right)} \underset{E_{\mathrm{b}} \rightarrow 0}{\sim} 0 .
$$

The corrections neglected on the left hand side of Eq. (50) are all linear in $E_{\mathrm{b}}$, while the leading contribution involving the momentum integral is proportional to $\sqrt{\left|E_{\mathrm{b}}\right|}$ in the limit $E_{\mathrm{b}} \rightarrow 0$. In particular, the asymptotic behaviour of the integral can be determined explicitly via a change of variable in spherical coordinates from the modulus of the momentum, $p$, to the dimensionless quantity $p / \sqrt{m\left|E_{\mathrm{b}}\right|}$. This yields:

$$
\int d \mathbf{p} \frac{\left|\left\langle\phi_{\text {res }}|W| \phi_{\mathbf{p}}^{(+)}\right\rangle\right|^{2}}{p^{2}\left(p^{2}+m\left|E_{\mathrm{b}}\right|\right)} \underset{E_{\mathrm{b}} \rightarrow 0}{\sim} \frac{2 \pi^{2}\left|\left\langle\phi_{\text {res }}|W| \phi_{0}^{(+)}\right\rangle\right|^{2}}{\left(m\left|E_{\mathrm{b}}\right|\right)^{1 / 2}} .
$$

Equation (40) may be used to eliminate the matrix element involving the coupling, $W(r)$, on the right hand side of Eq. (51) in favour of the product $a_{\mathrm{bg}} \Delta B \mu_{\text {res }}$. The solution of Eq. (50) with respect to the bound state energy thus reads:

$$
E_{\mathrm{b}}=-\frac{\hbar^{2}}{m\left[-a_{\mathrm{bg}} \Delta B /\left(B-B_{0}\right)\right]^{2}} .
$$

As anticipated (Donley et al., 2002) for the general reasons outlined in Section [1] this formula exactly recovers Eq. (1) in the limit $B \rightarrow B_{0}$, as the singular contribution to $a(B)$ on the right hand side of Eq. (39) exceeds $\left|a_{\mathrm{bg}}\right|$.

\section{The Wigner threshold law}

The preceding derivation of the low binding energy behaviour of the right hand side of Eq. (47) may be extended also to positive continuum energies, $E=\hbar^{2} k^{2} / m$, which determines the width of the Feshbach resonance level (Mies et al., 2000; Moerdiik et al., 1995). This width in energy is related to the decay rate of the bare resonance state into a given continuum level associated with the wave number $k$ via Fermi's golden rule (Dürr et al., 2004b; Góral et al., 2004; Haque and Stoof, 2005; Mukaiyama et al., 2003). The approach underlying these derivations is based on the spectral decomposition of $G_{\mathrm{bg}}(z)$ in Eq. (49), in addition to the following formula:

$$
\frac{1}{z-p^{2} / m}=-i \pi \delta\left(E-p^{2} / m\right)+\mathcal{P} \frac{1}{E-p^{2} / m} .
$$

Here " $z=E+i 0$ " is the complex argument of $G_{\mathrm{bg}}(z)$ introduced in Eq. (26), and $\mathcal{P}$ indicates the principal value of the momentum integral in Eq. (49). In accordance with Fermi's golden rule, the width of the resonance level is given by the modulus of the imaginary part of the following matrix element of the bare entrance channel Green's function:

$$
\left\langle\phi_{\text {res }}\left|W G_{\mathrm{bg}}(z) W\right| \phi_{\mathrm{res}}\right\rangle \underset{k \rightarrow 0}{\sim} \mu_{\mathrm{res}}\left(B_{\mathrm{res}}-B_{0}-i k a_{\mathrm{bg}} \Delta B\right) .
$$

We note, however, that Eq. (53) is not suitable for determining the left hand side of Eq. (54) in the case of negative bound state energies, $z=E_{\mathrm{b}}$, as the $\delta$ function contribution vanishes identically. The derivation of Eq. (52) therefore relies upon Eq. (48).

As the imaginary part of Eq. (54) is associated with the decay of the bare resonance state, the product $a_{\mathrm{bg}} \mu_{\text {res }} \Delta B$ is always positive and characterises the strength of the interchannel coupling. Equation (54) also determines the low energy dressed continuum wave functions of Eqs. (32) and (33) via Eq. (34). In particular, the $s$-wave scattering amplitude of Eqs. (37) and (38) is given by its well known general expansion about zero momentum (Taylor, 1972),

$$
f_{0}(\hbar k) \underset{k \rightarrow 0}{\sim}-a(1-i k a),
$$

which is applicable provided that the condition $k a \ll 1$ is fulfilled. The proportionality to $k=\sqrt{m E} / \hbar$ of the imaginary parts on the right hand sides of Eqs. (54) and (55) reflects a general prediction by Wigner, known as the threshold law (Wigner, 1948). In the context of an analytic continuation to imaginary wave numbers, $k=i \sqrt{m\left|E_{\mathrm{b}}\right|} / \hbar$, Eq. (54) also yields the universal bound state energy of Eq. (52) using Eq. (47) (Duine and Stoof, 2004). The universal regime may therefore be considered as the range of magnetic field strengths in which the Wigner threshold law applies to the energy of the Feshbach molecule in Eq. (47). Its extension about $B_{0}$ depends on the interplay between the spin exchange (or dipolar) and entrance channel interactions as well as on the properties of the Feshbach resonance state (Köhler et al., 2003b; Marcelis et al., 2004; Mies et al., 2000; Nygaard et al., 2006).

Figure 12, for instance, illustrates several theoretical approaches to the bound state energy of the ${ }^{85} \mathrm{Rb}_{2}$ Feshbach molecule in the vicinity of the $155 \mathrm{G}$ zero energy resonance. Although the universal formula of Eq. (1) determines the asymptotic behaviour of $E_{\mathrm{b}}$ in the limit $B \rightarrow B_{0}$, it provides a reasonable approximation only in a small region between $B_{0} \approx 155 \mathrm{G}$ and about $158 \mathrm{G}$. Both the full coupled channels calculation (circles) and the two-channel predictions (solid curve) depend on specific properties of the particular ${ }^{85} \mathrm{Rb}$ zero energy resonance besides the scattering length. These approaches fully recover the measured Feshbach molecular energies (see the inset of Fig. 12) over the entire experimental range of magnetic field strengths from about $156 \mathrm{G}$ to $162 \mathrm{G}$ (Claussen et al., 2003). The slope of the linear magnetic field dependence of $E_{\mathrm{b}}$ in the limit of high fields in Fig. 12 is determined by the bare resonance energy, $E_{\text {res }}(B)$ (dot-dashed line). This indicates an increasing admixture of the closed channel spin configuration to the dressed bound state.

\section{Closed channel admixture}

In accordance with Eq. (45) and the unit normalisation of the bare Feshbach resonance state, the closed channel admixture to the Feshbach molecule is determined by the wave function normalisation constant $\mathcal{N}_{\mathrm{b}}$ of Eq. (46) to be:

$$
\left\langle\phi_{\mathrm{b}}^{\mathrm{cl}} \mid \phi_{\mathrm{b}}^{\mathrm{cl}}\right\rangle=\mathcal{N}_{\mathrm{b}}^{-2} 4 \pi \int_{0}^{\infty} r^{2} d r\left|\phi_{\text {res }}(r)\right|^{2}=\mathcal{N}_{\mathrm{b}}^{-2} .
$$




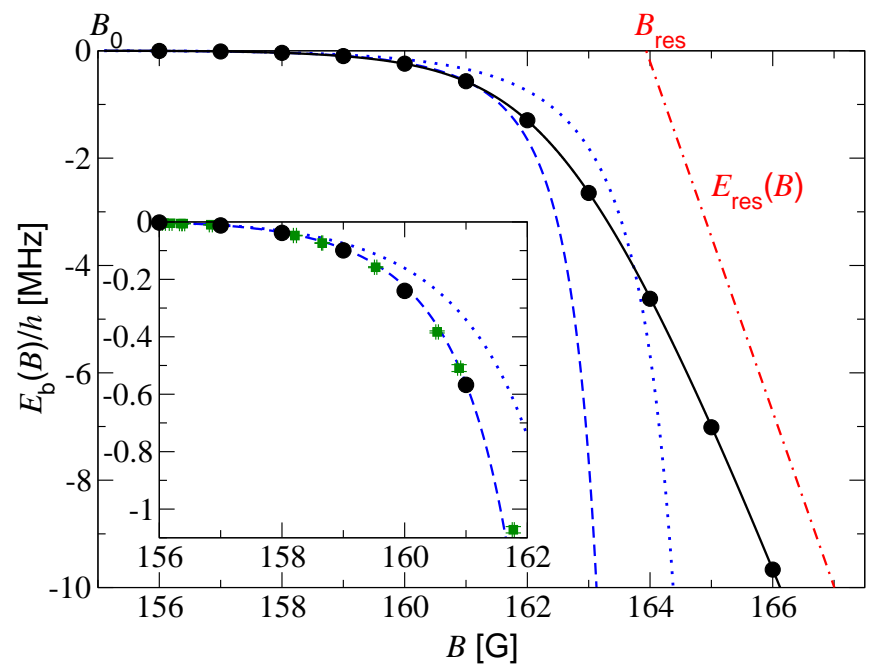

FIG. 12 (Colour in online edition) The bound state energy of the ${ }^{85} \mathrm{Rb}_{2}$ Feshbach molecule as a function of the magnetic field strength in the vicinity of the $155 \mathrm{G}$ zero energy resonance. The circles indicate a full coupled channels calculation (Kokkelmans, 2002). The solid curve results from a two-channel approach (Góral et al., 2005). For comparison, the dotted curve represents the universal estimate of $E_{\mathrm{b}}$, while the dashed curve includes the leading correction to Eq. (1) due to the van der Waals tail of the background scattering potential (Gribakin and Flambaum, 1993; Köhler et al., 2003a) given by Eq. (66) of Subsection III.F We note the pronounced shift of about $9 \mathrm{G}$ between the measurable resonance position, $B_{0}$, and the magnetic field strength $B_{\text {res }}$ at which the resonance energy (dotdashed line) crosses the dissociation threshold of the entrance channel. The inset compares the theoretical approaches with measurements (Claussen et al., 2003) indicated by squares.

In the context of field theoretic approaches to the manybody physics of cold gases, the inverse square of the normalisation constant, $\mathcal{N}_{\mathrm{b}}^{-2}$, is sometimes referred to as the wave function renormalisation constant $Z(B)$ (Braaten et al., 2003; Bruun and Pethick, 2004; Duine and Stoof, 2003a; Góral et al., 2004). The quantity $Z(B)$ can be expressed in terms of the difference in magnetic moments of the Feshbach molecular state and a pair of separated atoms in the entrance channel spin configuration, i.e. $\partial E_{\mathrm{b}} / \partial B$, via the following formula:

$$
Z(B)=\mathcal{N}_{\mathrm{b}}^{-2}=\mu_{\mathrm{res}}^{-1} \frac{\partial E_{\mathrm{b}}}{\partial B} .
$$

This exact result follows directly from Eqs. (46) and (47) using the general relation

$$
G_{\mathrm{bg}}^{2}\left(E_{\mathrm{b}}\right)=-\frac{\partial}{\partial E_{\mathrm{b}}} G_{\mathrm{bg}}\left(E_{\mathrm{b}}\right)
$$

Within the universal regime of magnetic field strengths the closed channel admixture to the Feshbach molecule can therefore be inferred from the derivative of Eq. (1) with respect to $B$. This yields:

$$
Z(B) \underset{B \rightarrow B_{0}}{\sim} \frac{2 a \hbar^{2} /\left(m a^{2}\right)}{\mu_{\mathrm{res}} \Delta B a_{\mathrm{bg}}} .
$$

Here the limit $B \rightarrow B_{0}$ is performed on the side of positive scattering lengths of the zero energy resonance.

In accordance with Eq. (59), the wave function normalisation constant, $\mathcal{N}_{\mathrm{b}}$, diverges as the magnetic field strength approaches $B_{0}$, i.e. in the limit $a \rightarrow \infty$, due to the proportionality of its leading contribution to $\sqrt{a}$. This implies that the closed channel admixture to the Feshbach molecule of Eq. (56) is negligible in the universal regime and vanishes at the measurable zero energy resonance position. Within this limited range of magnetic field strengths about $B_{0}$ the Feshbach molecule can therefore be described in terms of just its entrance channel component, in analogy to Section [I] In particular, the wave function $\phi_{\mathrm{b}}^{\mathrm{bg}}(\mathbf{r})$ is determined by Eq. (2) at inter-atomic distances large compared to the van der Waals length of Fig. 11. In accordance with Eq. (3), the mean distance between the atomic constituents of universal Feshbach molecules is well estimated by its asymptotic value of one half of the scattering length in the limit $a \rightarrow \infty$ (Köhler et al., 2003b). In the universal regime of magnetic field strengths the Feshbach molecules are therefore proper diatomic halo states with all the general properties described in Section

\section{E. Experimental signatures of universality}

The suppression of the closed channel admixture to the Feshbach molecular state has been directly observed for the example of near resonant ${ }^{6} \mathrm{Li}_{2}$ dimers (Partridge et al., 2005). Figure 13 shows a comparison of these measurements with the universal estimate of Eq. (59) as well as with Eq. (68) of Subsection III.F. The estimate of Eq. (68) is based on Eq. (57) using the approximation for $E_{\mathrm{b}}$ of Eq. (66), which is illustrated in the inset of Fig. 12, The underlying approach (Gribakin and Flambaum, 1993) accounts for the corrections to the universal bound state energy of Eq. (11) due to the van der Waals interaction of the background scattering potential in addition to the scattering length $a(B)$. Its predictions agree well with exact coupled channels calculations of the closed channel admixture to the Feshbach molecular state (Partridge et al., 2005). Several experiments to date have also directly confirmed the universal limit of the binding energy, in analogy to the inset of Fig. 12 (Claussen et al., 2003; Donley et al., 2002), for a variety of alkali atomic species (Bartenstein et al., 2005; Moritz et al., 2005; Regal et al., 2003a).

\section{Collisional relaxation}

Besides these demonstrations of universality, the long range nature of highly excited Feshbach molecules is manifest in their lifetimes with respect to deeply inelastic collisions. Such molecular loss may occur due to relaxation into tightly bound diatomic states upon collisions with surrounding atoms or dimers (Cubizolles et al., 2003; Mukaiyama et al., 2003; Regal et al., 2004a). The associated loss mechanism was discussed first, in the context of cold gases, for collisions between $\mathrm{H}_{2}$ dimers and hydrogen atoms Balakrishnan et al., 


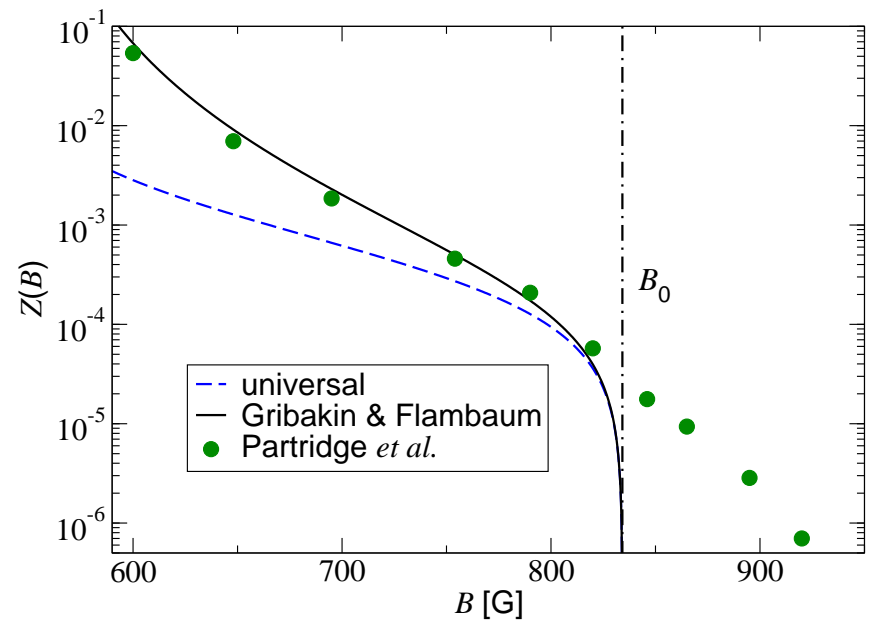

FIG. 13 (Colour in online edition) Occupation of the closed channel resonance level relative to the number of atom pairs in a balanced mixture of two spin components of ${ }^{6} \mathrm{Li}$ versus the magnetic field strength in the vicinity of the $834 \mathrm{G}$ zero energy resonance (Partridge et al., 2005). On the low field side of the resonance position $B_{0}$ (dot-dashed line) the gas was prepared as a Bose-Einstein condensate of ${ }^{6} \mathrm{Li}_{2}$ Feshbach molecules. The experimental data (circles) indicate the suppression of the closed channel admixture to the dressed molecular state, i.e. $Z(B)=\mathcal{N}_{\mathrm{b}}^{-2}$, over three orders of magnitude in agreement with Eq. (68) of Subsection 11 .F (solid curve). The dashed curve shows the universal estimate of Eq. 59 for comparison. We note that the bond length of the Feshbach molecules of Eq. (3) reaches the order of magnitude of the average inter-atomic spacing of the gas of $10^{4} a_{\text {Bohr }}$ at a magnetic field strength of about $800 \mathrm{G}$. In this strongly interacting regime the purely two-body theories are bound to break down. On the high field side of $B_{0}$ the remnant small resonance state population indicates pairing phenomena in a cold, strongly correlated two spin component Fermi gas with a negative scattering length (Partridge et al., 2005).

1997). In these threshold-less reactions the energy lost through de-excitation is transferred to the relative motion of the products in accordance with momentum conservation. The associated relative velocities are sufficiently high for the scattered particles to leave an atom trap. The density $n_{\mathrm{d}}$ of dimer molecules in a homogeneous gas is therefore depleted in accordance with the following rate equation:

$$
\dot{n}_{\mathrm{d}} / n_{\mathrm{d}}=-K_{\mathrm{ad}} n_{\mathrm{a}}-K_{\mathrm{dd}} n_{\mathrm{d}} .
$$

Here $n_{\mathrm{a}}$ is the density of atoms and $K_{\mathrm{ad}}$ and $K_{\mathrm{dd}}$ denote the inelastic loss rate constants associated with atom-dimer and dimer-dimer collisions, respectively.

Several theoretical studies of collisional relaxation of alkali metal systems have been performed in the limit of low vibrational excitation of the initial dimer states (Cvitas et al., 2002, $2005 \mathrm{a}$ b) as well as for Feshbach molecules (Petrov et al., 2004, 2005b). In the case of collisions between alkali metal atoms and their dimers in excited states, whose bond lengths are smaller than $l_{\mathrm{vdW}}$, ab initio calculations suggest the inelastic rate constants, $K_{\mathrm{ad}}$, to be on the order of $10^{-10} \mathrm{~cm}^{3} / \mathrm{s}$ (Cvitas et al., 2002, 2005a b). For such species the collisional relaxation rates in cold gases would be too large for the observation of any phenomena relying upon the equilibration of the molecular component. The expected short lifetimes of alkali dimers have been confirmed, for instance, via photo-association of ${ }^{87} \mathrm{Rb}$ atoms in a Bose-Einstein condensate (Wynar et al., 2000).

\section{Lifetime of Feshbach molecules in Fermi gases}

Therefore it came as a surprise that cold Feshbach molecules produced from incoherent mixtures of two spin components of fermionic atoms could be stabilised for up to several seconds at densities of about $10^{13}$ atoms $/ \mathrm{cm}^{3}$ (Cubizolles et al., 2003; Jochim et al., 2003a; Regal et al., 2004a; Strecker et al., 2003). Under such conditions the ratio of elastic to inelastic collisions is sufficiently large to allow for an efficient evaporative cooling of the dimers. As a consequence of their stability, even the Bose-Einstein condensation of Feshbach molecules has been observed in the vicinity of the broad zero energy resonances of ${ }^{6} \mathrm{Li}$ and ${ }^{40} \mathrm{~K}$ at about $830 \mathrm{G}$ and $202 \mathrm{G}$, respectively (Greiner et al., 2003; Jochim et al., 2003b; Zwierlein et al., 2003). The associated resonance widths, $\Delta B$, are on the order of $300 \mathrm{G}$ for ${ }^{6} \mathrm{Li}$ (Bartenstein et al., 2005) and about $8 \mathrm{G}$ in the case of ${ }^{40} \mathrm{~K}$ (Greiner et al., 2003). Such a broadness provides the opportunity of performing measurements in the universal regime of magnetic field strengths.

Both species of dimers in these experiments consist of pairs of unlike fermionic atoms in the lowest energetic Zeeman states. In the case of ${ }^{40} \mathrm{~K}$ the associated atomic spin components are determined by the quantum numbers $\left(f=9 / 2, m_{f}=\right.$ $-9 / 2)$ and $\left(f=9 / 2, m_{f}=-7 / 2\right)$. Here $f$ labels the angular momentum quantum number of the hyperfine level with which the Zeeman state correlates adiabatically at zero magnetic field, and $m_{f}$ indicates its spin orientation with respect to the field axis. Similarly, the pair of atomic Zeeman states associated with the $830 \mathrm{G}$ zero energy resonance of ${ }^{6} \mathrm{Li}$ is described by the quantum numbers $\left(f=1 / 2, m_{f}=1 / 2\right)$ and $\left(f=1 / 2, m_{f}=-1 / 2\right)$. We note that the Pauli exclusion principle allows such atom pairs to interact via $s$ waves given that their spin wave functions are anti-symmetric. Consequently, all the results of Section $\amalg$ on isotropic halo dimers apply to these Feshbach molecules consisting of unlike fermions. All experiments on molecular Bose-Einstein condensation have been performed at near resonant magnetic field strengths for which the associated dimer bond lengths can be as large as $100 \mathrm{~nm}$ (Zwierlein et al., 2003).

The stability of such Feshbach molecules with respect to collisional relaxation relies upon the Pauli exclusion principle in addition to the separation of length scales associated with the initial and final states (Petrov et al., 2004, 2005b). In accordance with Eq. (3), the spatial extent of the initial halo wave function is determined by the scattering length, $a$. The bond length of the final, deeply bound target level may be estimated by the van der Waals length, $l_{\mathrm{vdW}}$, which is much smaller than $a$. Both dimer-dimer and atom-dimer relaxation therefore require at least three fermions to come together at short distances on the order of $l_{\mathrm{vdW}}$. One pair among these 
atoms necessarily shares the same spin state and can interact at most via $p$ waves. As the momentum scale associated with the initial Feshbach molecular state is determined by the wave number $k \sim 1 / a$, the inelastic loss rate constants are suppressed by powers of $k l_{\mathrm{vdW}} \ll 1$.

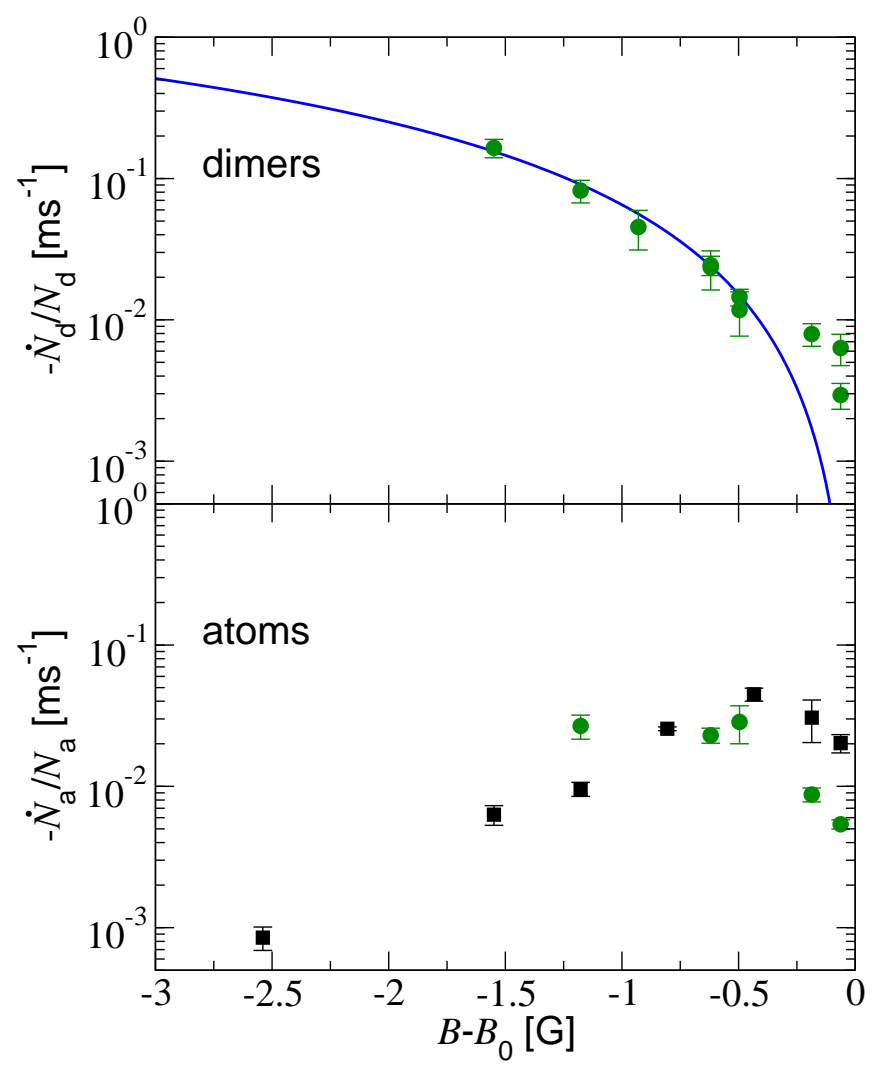

FIG. 14 (Colour in online edition) Loss rates of diatomic Feshbach molecules (upper panel) and remnant atoms (lower panel) versus the magnetic field detuning, $B-B_{0}$, in the vicinity of the $202 \mathrm{G}$ zero energy resonance in a cold gas of ${ }^{40} \mathrm{~K}$ with a peak density of about $1.5 \times 10^{13}$ atoms $/ \mathrm{cm}^{3}$ (Regal et al. , 2004a). The dimers were produced using the technique of adiabatic sweeps of the magnetic field strength described in Section IV] Their number is denoted by $N_{\mathrm{d}}$, while $N_{\mathrm{a}}$ refers to the number of remnant unbound atoms. The circles in the upper panel indicate the measured dimer loss, whose general trend is well fit by an $a^{-2.3 \pm 0.4}$ power law as a function of the scattering length (solid curve). Significant deviations from this scattering length dependence occur in a small region of magnetic field strengths where the bond length of the dimers of Eq. (3) is comparable to the average distance of the atoms in the gas. The lower panel shows the loss rate of remnant unbound atoms in gases with (circles) or without (squares) deliberate production of Feshbach molecules. The associated trends suggest that the atomic loss is largely unaffected by the presence of a dimer component in the gas (Regal et al., 2004a).

Based on the halo wave function of Eq. (2), the precise $a$ dependences of the suppression factors for atom-dimer and dimer-dimer relaxation have been predicted to be $a^{-3.33}$ and $a^{-2.55}$, respectively (Petrov et al., 2004, 2005b). While these predictions strictly apply just to the universal regime of magnetic field strengths, Fig.14 illustrates that their general trends agree with measurements on cold gases of ${ }^{40} \mathrm{~K}$ with a component of Feshbach molecules (Regal et al., 2004a). These ob- servations are consistent with the reduction of a predominant dimer-dimer relaxation in the limit of large scattering lengths, where the lifetimes reach about $100 \mathrm{~ms}$. Conversely, as the magnetic field strength is tuned away from the zero energy resonance, the molecular lifetimes approach those small values of less than a millisecond, typical for short ranged alkali dimers in cold gases.

Besides their scaling properties with respect to the scattering length, the inelastic loss rate constants of Feshbach molecules depend sensitively on inter-atomic interactions at short distances below $l_{\mathrm{vdw}}$. The experimental trends regarding the collisional relaxation of alkali dimers confined to such length scales are inconclusive. Remarkably long lifetimes on the order of seconds were reported, for instance, even in the case of short ranged, closed-channel dominated Feshbach molecules produced in a fermionic ${ }^{6} \mathrm{Li}$ gas in the vicinity of the narrow $543 \mathrm{G}$ zero energy resonance (Strecker et al., 2003). Such alkali dimers, however, are not described by the halo wave function of Eq. (2). Their observed stability, therefore, suggests a mechanism for the suppression of collisional relaxation beyond the scaling of the associated loss rate constants with powers of the inverse scattering length.

\section{Lifetime of Feshbach molecules in Bose gases}

Feshbach molecules consisting of identical Bose atoms generally tend to be less stable than their fermionic counterparts. Large collisional relaxation rate constants on the typical order of $10^{-10} \mathrm{~cm}^{3} / \mathrm{s}$ have been reported for those dimers associated in cold gases in the vicinity of comparatively narrow zero energy resonances of ${ }^{87} \mathrm{Rb},{ }^{133} \mathrm{Cs}$ and ${ }^{23} \mathrm{Na}$ (Dürr et al., 2004a; Herbig et al., 2003; Mukaiyama et al., 2003; Yurovsky et al., 1999b, 2000). With a width of about $11 \mathrm{G}$ (Claussen et al., 2003) the $155 \mathrm{G}$ zero energy resonance of ${ }^{85} \mathrm{Rb}$ is by far the broadest among the bosonic species from which Feshbach molecules were produced. The atomic constituents of these dimers are prepared in the excited Zeeman state determined by the quantum numbers $\left(f=2, m_{f}=-2\right)$ which, in contrast to the electronic ground state, can be magnetically trapped. Pairs of such atoms are subject to inelastic spin relaxation collisions involving the threshold-less transition of the atomic energy level to a deeper Zeeman state (Roberts et al., 2000b). The associated $d$-wave exit channels are illustrated in Table [II. It is the spontaneous dissociation via spin relaxation, rather than collisional relaxation of the molecular vibrational state, that predominantly limits the observed lifetimes of Feshbach molecules produced in dilute gases of ${ }^{85} \mathrm{Rb}$. This decay mechanism has been unequivocally identified through comparisons with quantitative predictions (Köhler et al., 2005; Thompson et al., 2005b).

Figure 15 shows the monotonic increase of the measured lifetimes over three orders of magnitude as the magnetic field strength approaches the resonance position, $B_{0}$. The lifetime of these Feshbach molecules is density independent and reaches several tens of milliseconds. This behaviour of the molecular decay can be explained in terms of a probabilistic estimate based on the average volume, $\mathcal{V}=4 \pi\left\langle r^{3}\right\rangle / 3$, occu- 


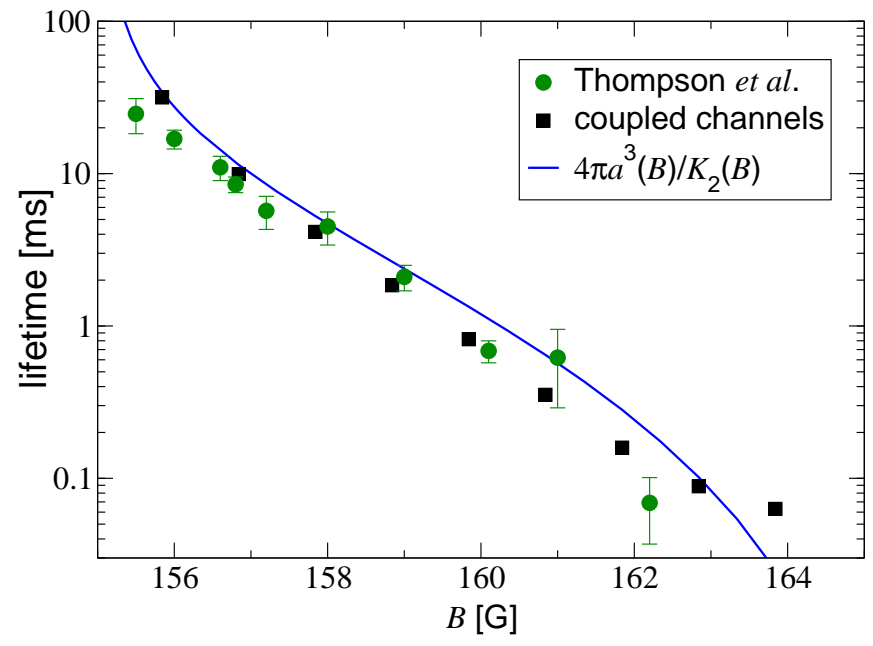

FIG. 15 (Colour in online edition) The lifetime of ${ }^{85} \mathrm{Rb}_{2}$ dimers versus magnetic field strength in the vicinity of the $155 \mathrm{G}$ zero energy resonance. The circles indicate measurements in a cold thermal gas with a peak density of $6.6 \times 10^{11}$ atoms $/ \mathrm{cm}^{3}$ (Thompson et al., 2005b), while the squares refer to exact coupled channels calculations accounting for the spontaneous dissociation of Feshbach molecules due to spin relaxation (Köhler et al., 2005). The solid curve represents an asymptotic estimate of the lifetime based on the universal halo wave function of Eq. (2) and the spin relaxation loss rate constant, $K_{2}(B)$, associated with a non-degenerate Bose gas in the limit of zero collision energy. The two-body theory fails only close to resonance when the bond length of the molecules becomes comparable to the average inter-atomic distance of the gas.

pied by an atom pair in the universal halo state of Eq. (2). In analogy to Eq. (3) this volume is determined in terms of the scattering length $a$ by the formula $\mathcal{V}=\pi a^{3}$. The event rate, $\gamma$, associated with the spin relaxation of such a bound atom pair may be expressed in terms of the loss rate constant $K_{2}$ for spin relaxation in a non-degenerate Bose gas (Roberts et al., 2000b). This yields $\gamma=K_{2} /(4 \mathcal{V})$ (Stoof et al., 1989). The lifetime, $\tau=1 / \gamma$, is therefore proportional to the ratio of the magnetic field dependent quantities $a^{3}(B)$ and $K_{2}(B)$, i.e., $\tau=4 \pi a^{3}(B) / K_{2}(B)$, where, near resonance, $K_{2}(B)$ can be evaluated at zero collision energy. The same formula for the molecular lifetime also follows rigorously from Fermi's golden rule (Köhler et al., 2005) and exactly recovers the results of the full coupled channels calculations of Fig. 15 in the limit $B \rightarrow B_{0}$. The magnetic field dependence of $K_{2}(B)$ can be inferred from general properties of inelastic collisions (Bohn and Julienne, 1997, 1999). Consequently, the stability of Feshbach molecules in both two spin component Fermi gases of ${ }^{40} \mathrm{~K}$ and dilute vapours of ${ }^{85} \mathrm{Rb}$ directly probes the halo nature of these dimers near resonance, despite their different decay mechanisms.

\section{F. Classification of zero energy resonances}

The singular behaviour of the scattering length as well as the associated existence of a universal regime of magnetic field strengths are common to all Feshbach resonance phe- nomena in cold gases. Two-body universality implies, in particular, that the long range properties of the dressed diatomic bound states are well described solely in terms of an effective entrance channel interaction. The range of validity of such single channel approaches provides classification schemes for zero energy resonances.

\section{Size of the universal regime}

The halo wave function of Eq. (2) may be interpreted as the only bound state supported by a contact pseudo potential, $g \delta(\mathbf{r})$, describing the low energy spectrum of an atom pair with a positive scattering length. Due to their simplicity, contact interactions are widely used in theoretical descriptions of cold gases (Dalfovo et al., 1999; Dalibard, 1999; Randeria, 1995). In these approaches the properties of inter-atomic collisions enter the many-body Hamiltonian in terms of a coupling constant, $g=4 \pi \hbar^{2} a / m$, depending on the microscopic potential just through the scattering length, $a$. The associated binary $s$ wave scattering amplitude is given by the following formula:

$$
f_{0}(\hbar k)=-a /(1+i k a)
$$

We note that Eq. (61) also recovers the general low wave number expansion of Eq. (55) in the Wigner threshold law regime. Consequently, the contact interaction approach provides a minimal implementation of the universal low energy two-body physics, applicable to both positive and negative scattering lengths. In the latter case the contact pseudo potential does not support any bound state.

The assumption of universality of the Feshbach molecular state implies the closed channel admixture of Eq. (56) to be negligible. For magnetic field strengths, $B$, far inside the width of the zero energy resonance, i.e. $\left|B-B_{0}\right| \ll \Delta B$, Eqs. (39) and (59) therefore yield the following condition necessary for the applicability of the contact interaction approach:

$$
\left|\frac{B-B_{0}}{\Delta B}\right| \ll \frac{\left|\mu_{\mathrm{res}} \Delta B\right|}{2 \hbar^{2} /\left(m a_{\mathrm{bg}}^{2}\right)} .
$$

The energy ratio on the right hand side of Eq. (62) provides an upper estimate for the extent of the universal regime relative to the width of the zero energy resonance. A ratio small compared to unity indicates a weak coupling between the closed and entrance channels. Conversely, broad zero energy resonances with a large product $\left|\mu_{\text {res }} \Delta B\right|$ tend to favour negligible admixtures of the bare resonance level to the dressed Feshbach molecular state over a substantial fraction of their width. This trend may be enhanced by a small energy $\hbar^{2} /\left(m a_{\mathrm{bg}}^{2}\right)$ which indicates the presence of bound vibrational $\left(a_{\mathrm{bg}}>0\right)$ or virtual $\left(a_{\mathrm{bg}}<0\right)$ near resonant energy levels of the bare background scattering potential.

A small closed channel admixture to the Feshbach molecular state, however, is not necessarily identical to the universality of the binding energy. While for the example of the comparatively weakly coupled $1007 \mathrm{G}$ zero energy resonance of ${ }^{87} \mathrm{Rb}$ (Dürr et al., 2004b) the right hand side of Eq. (62) gives 
0.1 , it is as large as 81 in the case of the $155 \mathrm{G}$ zero energy resonance of ${ }^{85} \mathrm{Rb}$. The estimate of Eq. (62) for ${ }^{85} \mathrm{Rb}$, however, is inaccurate with respect to the extension of the universal regime in units of the width, $\Delta B$, given the results of Fig. 12. The inset of Fig. 12]reveals that this inaccuracy originates predominantly from corrections to the universal binding energy due to the van der Waals tail of the bare ${ }^{85} \mathrm{Rb}$ background scattering potential (Gribakin and Flambaum, 1993). In the case of such entrance-channel dominated zero energy resonances even the lowest order corrections to universality may be described just by a single channel approach (Köhler et al., 2003a). The minimal requirements on the associated effective potential, $V(B, r)$, besides its long range asymptotic behaviour of Eq. (5) are closely related to general properties of alkali dimer energy wave functions.

\section{Entrance-channel dominated resonances}

In accordance with Fig. 11 the vibrational bound state wave functions of ${ }^{85} \mathrm{Rb}_{2}$ Feshbach molecules consist of short and long range contributions. The characteristic scale for such a spatial separation is the van der Waals length of Eq. (7). Due to the deep wells of realistic background scattering potentials, the entrance channel wave functions of alkali dimers are well described by the semi-classical Wentzel-Kramers-Brillouin (WKB) approximation at short inter-atomic distances, $r \ll$ $l_{\mathrm{vdW}}$. Their behaviour at large separations, $r \gg l_{\mathrm{vdW}}$, is determined mainly by the van der Waals interaction of Eq. (5) in addition to the energy of the state. In both spatial regions the functional forms of the associated asymptotic solutions of the radial Schrödinger equation are known analytically (Gao, 1998a b; Gribakin and Flambaum, 1993). As the interactions between alkali atom pairs are dominated by large van der Waals coefficients, $C_{6}$, on the order of thousands of atomic units (the atomic unit of $C_{6}$ is $9.5734 \times 10^{-26} \mathrm{~J} \mathrm{~nm}^{6}$ ), the asymptotic wave functions can be matched. Such a matching procedure provides the basis of accurate semi-classical treatments of bound as well as continuum entrance channel wave functions (Flambaum et al., 1999; Gribakin and Flambaum, 1993).

Provided that the interaction of a pair of alkali atoms is well described by an effective entrance channel potential, $V(B, r)$, the semi-classical approach to the zero energy wave function determines the scattering length to be (Gribakin and Flambaum, 1993):

$$
a=\bar{a}\left[1-\tan \left(\varphi_{\mathrm{WKB}}-\pi / 8\right)\right] .
$$

Here $\bar{a}$ is usually referred to as the mean scattering length and $\varphi_{\mathrm{WKB}}$ is the semi-classical phase shift. In accordance with the WKB approach, $\varphi_{\mathrm{WKB}}$ consists of the following integral between the zero energy classical turning point, $r_{0}$, associated with the effective potential, $V(B, r)$, and infinite distances:

$$
\varphi_{\mathrm{WKB}}=\frac{1}{\hbar} \int_{r_{0}}^{\infty} d r \sqrt{-m V(B, r)}
$$

While $\varphi_{\mathrm{WKB}}$ is thus sensitive to the entire well of the interaction, from $V\left(B, r_{0}\right)=0$ to its long range tail of Eq. (5), the coefficient $\bar{a}$ of Eq. (63) depends just on the van der Waals length, $l_{\mathrm{vdW}}$. Its explicit expression in terms of $l_{\mathrm{vdW}}$ and Euler's $\Gamma$ function reads:

$$
\bar{a}=\frac{l_{\mathrm{vdW}}}{\sqrt{2}} \frac{\Gamma(3 / 4)}{\Gamma(5 / 4)} \approx 0.95598 \times l_{\mathrm{vdW}} .
$$

In accordance with Eq. (63), the parameter $\bar{a}$ determines the characteristic scale of the scattering length. This average potential range is modulated by the poles of the tangent function provided that its argument, $\varphi_{\mathrm{WKB}}-\pi / 8$, is close to an odd integer multiple of $\pi / 2$. As $\varphi_{\mathrm{WKB}}$ increases, each singularity of $a$ indicates the emergence of an additional vibrational bound state in the potential well.

The functional form of Eq. (63) using a realistic interaction is largely analogous to the formula for the scattering length of the simplified square well plus hard core model of an interatomic potential (Gribakin and Flambaum, 1993). In the case of this exactly solvable model the mean scattering length, $\bar{a}$, recovers the finite outer radius of the well. The analogy between these realistic and simplified interactions therefore implies that in the limit $a \gg \bar{a}$ the energy of the highest excited vibrational state is well approximated by the following asymptotic formula:

$$
E_{\mathrm{b}} \approx-\hbar^{2} /\left[m(a-\bar{a})^{2}\right] .
$$

An independent analysis based on effective range theory for realistic inter-atomic interactions (Flambaum et al., 1999; Gao, 1998a) confirms the magnitude of the range parameter $\bar{a}$ of Eq. (66) up to a constant factor on the order of unity (Gao, 2004). The remnant uncertainties associated with Eq. (66) may be related to the divergence of the effective range expansion for any potential with a long range van der Waals tail (Taylor, 1972). Figure 12 illustrates the accuracy of Eq. (66) within the experimentally relevant range of binding energies of near resonant ${ }^{85} \mathrm{Rb}_{2}$ Feshbach molecules.

According to the semi-classical approach, at magnetic field strengths in the vicinity of entrance-channel dominated zero energy resonances the diatomic bound and continuum spectra are determined mainly by $a(B)$ and $\bar{a}$. In such a case, any potential, $V(B, r)$, which at each magnetic field strength, $B$, accounts for the scattering length as well as the van der Waals tail of Eq. (5) provides a suitable description of the near resonant binary physics. This conclusion is rather intuitive because cold collisions are characterised by de Broglie wave lengths too large to resolve details of the effective entrance channel interaction besides its long range behaviour. The adjustment of $V(B, r)$ to recover the exact magnetic field dependence of the scattering length of Eq. (39) may be achieved, for instance, by varying the radius of its hard core. In the context of quantum defect theory such a description of alkali dimer spectra in terms of just the parameters $a$ and $C_{6}$ has been rigorously derived for a range of energies much wider than the cold regime (Gao, 1998a).

As $\bar{a}$ is positive, the semi-classical estimate of the bound state energy of Eq. (66) is always below the universal prediction of Eq. (1) and becomes singular in the limit $a(B) \rightarrow \bar{a}$, outside the range of validity of Eq. (66). This unphysical 
behaviour is counterbalanced in Eq. (47) by an increasing closed channel admixture to the Feshbach molecule, in accordance with Eq. (59), which tends to impose a linear slope on $E_{\mathrm{b}}(B)$. The principal question of the applicability of single channel approaches outside the universal regime of magnetic field strengths is decided by which one of these trends prevails near resonance (Köhler et al., 2004). Consequently, the bound state energy, $E_{\mathrm{b}}(B)$, in the vicinity of an entrancechannel dominated zero energy resonance is subject to the following inequality:

$$
\left|E_{\mathrm{b}}(B)+\frac{\hbar^{2}}{m[a(B)-\bar{a}]^{2}}\right|<\left|E_{\mathrm{b}}(B)+\frac{\hbar^{2}}{m a^{2}(B)}\right| .
$$

Within the range of validity of Eq. (66) the admixture of the closed channel resonance state to the Feshbach molecule is small compared to unity and can be determined from the binding energy via Eq. (57). This yields:

$$
Z(B)=\frac{2 a \hbar^{2} /\left(m a^{2}\right)}{\mu_{\mathrm{res}} \Delta B a_{\mathrm{bg}}} \frac{\left(1-a_{\mathrm{bg}} / a\right)^{2}}{(1-\bar{a} / a)^{3}} .
$$

The accuracy of Eq. (68) in applications to ${ }^{6} \mathrm{Li}_{2}$ Feshbach molecules in the vicinity of the entrance-channel dominated $834 \mathrm{G}$ zero energy resonance is illustrated in Fig. 13.

Based on Eq. 677) a more practical criterion can be derived from a low energy expansion of the right hand side of Eq. (47) using a specific implementation of the general two-channel approach (Góral et al., 2004). This yields a dimensionless parameter, $\eta$, whose smallness indicates the validity of Eq. (66) beyond the universal regime of magnetic field strengths (Stoll and Köhler, 2005). Consequently, an entrance-channel dominated zero energy resonance fulfils the condition

$$
\eta=\frac{\bar{a}}{a_{\mathrm{bg}}} \frac{\hbar^{2} /\left(m \bar{a}^{2}\right)}{\mu_{\mathrm{res}} \Delta B} \ll 1 .
$$

Such a criterion also results from an adiabatic description of Feshbach resonances (Petrov, 2004).

In the opposite limit, $\eta \gg 1$, a zero energy resonance shall be referred to, in the following, as closed-channel dominated. Closed-channel dominated zero energy resonances are typically narrow and their universal regime of magnetic field strengths is experimentally largely inaccessible. The description of their physical properties therefore crucially relies upon an explicit treatment of at least two scattering channels.

\section{G. Characteristic parameters of zero energy resonances}

For any two-channel approach to be sensible its implementation should describe the two-body energy spectrum beyond the Wigner threshold law domain. Otherwise the same physics could be captured simply by the contact pseudo interaction of Subsection III.F The approach should therefore recover both the scattering length of Eq. (39) and the binding energy of the Feshbach molecule beyond the universal regime. Even within such a comparatively wide range of energies a variety of inter-atomic potentials are capable of describing the same two-body physics. It is the objective of this subsection to provide a minimal set of physical parameters that every two-channel approach should account for, and to illustrate a practical implementation for a typical experimental situation. The adjustment of the Hamiltonian of Eq. (16) in the single resonance approach of Eq. (36) will be performed on the basis of the general form of its energy spectrum derived in Subsection III.C According to these derivations, the dressed two-channel energy states depend on the bare states associated with the background scattering via $G_{\mathrm{bg}}(z)$, on the resonance energy, $E_{\text {res }}(B)$, and on the product $W\left|\phi_{\text {res }}\right\rangle$ characterising the inter-channel coupling. Just these quantities need to be adjusted. The specific form of the resonance wave function, $\phi_{\text {res }}(r)$, does not affect the two-body spectrum in the single resonance approach.

\section{The background scattering potential}

In accordance with Subsection $\amalg$ III.F all implementations of $V_{\mathrm{bg}}(r)$ which recover $a_{\mathrm{bg}}$ in addition to Eq. (5) yield equivalent energy spectra beyond the cold regime (Gao, 1998a), provided that their number of levels is large compared to unity. A hard sphere in addition to the van der Waals tail of Eq. (5), for instance, provides such a background scattering potential with a minimal number of parameters. Its explicit expression reads:

$$
V_{\mathrm{bg}}(r)=\left\{\begin{array}{cl}
+\infty & : r<r_{0} \\
-C_{6} / r^{6} & : \quad r>r_{0}
\end{array} .\right.
$$

The associated background scattering length is given by the following exact formula (Gribakin and Flambaum, 1993):

$$
a_{\mathrm{bg}}=\bar{a}\left[1-\tan \left(\varphi_{\mathrm{WKB}}-3 \pi / 8\right)\right] .
$$

Here $\bar{a}$ is the mean scattering length of Eq. (65) and $\varphi_{\mathrm{WKB}}=$ $2 l_{\mathrm{vdW}}^{2} / r_{0}^{2}$ is the semi-classical phase shift of Eq. (64). The difference of $\pi / 4$ between the arguments of the tangent functions in Eqs. (63) and (71) is due to the discontinuity of Eq. (70) at the core radius $r_{0}$. Equation (71) may be used to determine $r_{0}$ in such a way that $V_{\mathrm{bg}}(r)$ of Eq. (70) exactly recovers the background scattering length. Similar procedures have been performed in two-channel approaches using continuous implementations of $V_{\mathrm{bg}}(r)$ (Köhler et al., 2003b; Marcelis et al., 2004; Mies et al., 2000; Nygaard et al., 2006). An example of such an effective interaction is illustrated in Fig. 10 in addition to its energy levels. Table IV provides the parameters $a_{\mathrm{bg}}$ and $C_{6}$ characterising the background scattering potential for several experimentally relevant zero energy resonances.

Equation (70) as well as those equivalent implementations of $V_{\mathrm{bg}}(r)$ that explicitly include the long range asymptotic van der Waals interaction of Eq. (5) are, in principle, suited to describe several bare energy levels. The number of bound states supported by Eq. (70), for instance, can be arbitrarily increased by decreasing the core radius $r_{0}$ under the constraint of a fixed background scattering length. The description of such wide energy ranges, however, is usually beyond the scope of two-channel approaches. In addition, 
TABLE IV Parameters characterising the background scattering potential associated with several experimentally relevant zero energy resonances. Those values of $a_{\mathrm{bg}}$ and $E_{-1}$ that are unreferenced refer to calculations performed for this review. The energy, $E_{-1}$, associated with the highest excited vibrational state of the background scattering potential is given only in the cases of isolated resonances. The atomic unit of the van der Waals dispersion coefficient, $C_{6}$, is $9.5734 \times 10^{-26} \mathrm{~J} \mathrm{~nm}^{6}, a_{\text {Bohr }}=0.052917 \mathrm{~nm}$ is the Bohr radius and $1 \mathrm{G}=10^{-4} \mathrm{~T}$.

\begin{tabular}{|c|c|c|c|c|}
\hline Species & $B_{0}[\mathrm{G}]$ & $a_{\mathrm{bg}}\left[a_{\mathrm{Bohr}}\right]$ & $C_{6}$ [a.u.] & $\left|E_{-1}\right| / h[\mathrm{MHz}]$ \\
\hline \multirow[t]{2}{*}{${ }^{6} \mathrm{Li}$} & 543.25(5) (Strecker et al., 2003) & 59 & 1393.39 (Yan et al., 1996) & \\
\hline & 834.149 (Bartenstein et al., 2005) & -1405 (Bartenstein et al., 2005) & 1393.39 (Yan et al., 1996) & \\
\hline \multirow[t]{2}{*}{${ }^{23} \mathrm{Na}$} & 853 (Stenger et al., 1999) & 63.9 (Mies et al., 2000) & 1561 (Kharchenko et al., 1997) & 208 \\
\hline & 907 (Stenger et al., 1999) & 62.8 (Mies et al., 2000) & 1561 (Kharchenko et al., 1997) & 218 \\
\hline \multirow[t]{2}{*}{${ }^{40} \mathrm{~K}$} & 202.10(7) (Regal et al., 2004c) & 174(7) (Loftus et al., 2002) & 3897 (Derevianko et al., 1999) & 8.6 \\
\hline & 224.21(5) (Regal and Jin, 2003) & 174(7) (Loftus et al., 2002) & 3897 (Derevianko et al., 1999) & 8.6 \\
\hline${ }^{85} \mathrm{Rb}$ & 155.0 (Thompson et al., 2005a) & -443(3) (Claussen et al., 2003) & 4703 (van Kempen et al., 2002) & 218 \\
\hline${ }^{87} \mathrm{Rb}$ & 1007.40(4) (Volz et al., 2003) & 100.5 (Volz et al., 2003) & 4703 (van Kempen et al., 2002) & 24.0 \\
\hline \multirow[t]{2}{*}{${ }^{133} \mathrm{Cs}$} & 19.90(3) (Chin et al., 2004b) & 163 (Julienne et al., 2004) & 6890(35) (Leo et al., 2000) & \\
\hline & 47.97(3) (Chin et al., 2004b) & 905 (Julienne et al., 2004) & 6890(35) (Leo et al., 2000) & 0.045 \\
\hline
\end{tabular}

the explicit treatment of the van der Waals tail of the background scattering potential is largely impractical in applications to the many-body physics of dilute gases. Several implementations of two-channel approaches, therefore, use effective low energy interactions to recover different aspects of the cold collision physics under the conditions of resonance enhancement (Bruun and Pethick, 2004; Chin, 2005; Drummond and Kheruntsyan, 2004; Duine and Stoof, 2004; Góral et al., 2004; Kokkelmans et al., 2002).

Figure 16 shows a typical range of energies relevant to the Stern-Gerlach separation of Feshbach molecules from a ${ }^{87} \mathrm{Rb}$ Bose-Einstein condensate (Dürr et al., 2004a). This experimental technique based on exposing a mixture of atoms and dimers to an inhomogeneous magnetic field (Chin et al., 2005; Dürr et al., 2004a; Herbig et al., 2003) is illustrated in Fig. 3 for the example of ${ }^{133} \mathrm{Cs}_{2}$. The relative force between the two components of the gas is proportional to the field gradient as well as to the difference in magnetic moments of a Feshbach molecule and a pair of separated atoms, i.e., $\partial E_{\mathrm{b}} / \partial B$. In the rubidium case the magnetic field dependence of $E_{\mathrm{b}}(B)$ in Fig. 16 is sensitive to the avoided crossing of dressed energy levels of ${ }^{87} \mathrm{Rb}_{2}$ due to the highest excited bare vibrational level of $V_{\mathrm{bg}}(r)$. In accordance with the size of the positive background scattering length of about $100 a_{\mathrm{Bohr}}$ (Volz et al., 2003), this level is sufficiently close to the dissociation threshold that its energy, $E_{-1}$, is directly probed by the experiment (Dürr et al., 2004a).

An effective entrance channel interaction suitable for a description of this experiment needs to recover both the precise value of $a_{\mathrm{bg}}$ and the bare vibrational level with the energy $E_{-1}$, beyond the Wigner threshold law regime. The associated minimal bare Green's function, $G_{\mathrm{bg}}(z)$, is therefore required to reproduce the bound state pole at the energy argument $z=E_{-1}$. Similarly to Eq. (30), this requirement leads to the following separable representation (Góral et al., 2004):

$$
G_{\mathrm{bg}}(z)=G_{0}(z)+G_{0}(z)\left|\chi_{\mathrm{bg}}\right\rangle \tau_{\mathrm{bg}}(z)\left\langle\chi_{\mathrm{bg}}\right| G_{0}(z)
$$

Here the form factor, $\left.\chi_{\mathrm{bg}}\right\rangle$, needs to recover, via the relation $G_{0}\left(E_{-1}\right)\left|\chi_{\mathrm{bg}}\right\rangle \propto\left|\phi_{-1}\right\rangle$, the bare vibrational state satisfying the
Schrödinger equation $H_{\mathrm{bg}}\left|\phi_{-1}\right\rangle=E_{-1}\left|\phi_{-1}\right\rangle$. The resonance term, $\tau_{\mathrm{bg}}(z)$, may be represented, by the following ratio:

$$
\tau_{\mathrm{bg}}(z)=\frac{\xi_{\mathrm{bg}}}{1-\xi_{\mathrm{bg}}\left\langle\chi_{\mathrm{bg}}\left|G_{0}(z)\right| \chi_{\mathrm{bg}}\right\rangle} .
$$

As $\tau_{\mathrm{bg}}(z)$ is required to reproduce the singularity of the bare Green's function in the limit $z \rightarrow E_{-1}$, the amplitude, $\xi_{\text {bg }}$, is given by $1 /\left\langle\chi_{\mathrm{bg}}\left|G_{0}\left(E_{-1}\right)\right| \chi_{\mathrm{bg}}\right\rangle$.

The separable representation of $G_{\mathrm{bg}}(z)$ of Eq. (72) provides the exact Green's function associated with an effective potential,

$$
V_{\mathrm{bg}}^{\mathrm{eff}}=\left|\chi_{\mathrm{bg}}\right\rangle \xi_{\mathrm{bg}}\left\langle\chi_{\mathrm{bg}}\right|
$$

determined by the amplitude, $\xi_{\mathrm{bg}}$, and the form factor, $\left\langle\chi_{\mathrm{bg}}\right\rangle$. This follows directly from the resolvent identity (Taylor, 1972), i.e.,

$$
G_{\mathrm{bg}}(z)=G_{0}(z)+G_{0}(z) V_{\mathrm{bg}}^{\mathrm{eff}} G_{\mathrm{bg}}(z)
$$

which is readily verified upon multiplication by $G_{\mathrm{bg}}^{-1}(z)=$ $z-H_{\mathrm{bg}}$ from the right and by $G_{0}^{-1}(z)=z+\hbar^{2} \nabla^{2} / m$ from the left. Iterating Eq. (75) yields the Born series which reduces to a geometric series in the special case of the separable potential of Eq. (74). Its exact sum is given by Eq. (72) with the resonance term of Eq. (73).

Such effective interactions are commonly employed in the theory of few-particle systems (Lovelace, 1964; Mitra, 1962; Yamaguchi, 1954) as well as in condensed matter physics (Schrieffer, 1964). In applications to cold gases the form factor is not resolved because it is sensitive only to the physics on distance scales on the order of the van der Waals length. Its associated functional form is therefore arbitrary and may be chosen, for instance, to be Gaussian (Góral et al., 2004). In the momentum space representation this yields:

$$
\left\langle\mathbf{p} \mid \chi_{\mathrm{bg}}\right\rangle=\chi_{\mathrm{bg}}(p)=\frac{\exp \left(-\frac{p^{2} \sigma_{\mathrm{bg}}^{2}}{2 \hbar^{2}}\right)}{(2 \pi \hbar)^{3 / 2}} .
$$




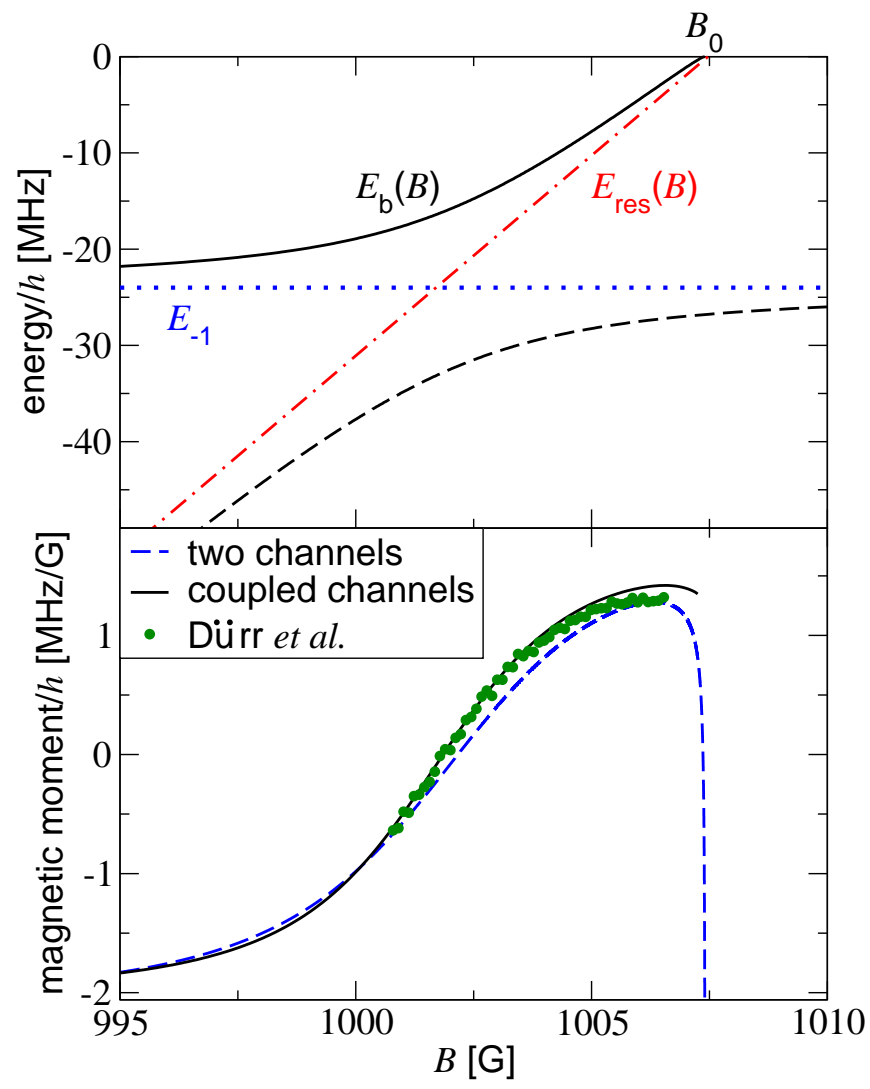

FIG. 16 (Colour in online edition) Avoided crossing of the highest excited vibrational levels of ${ }^{87} \mathrm{Rb}_{2}$ (upper panel) and the magnetic moment of the Feshbach molecule (lower panel) versus the magnetic field strength in the vicinity of the $1007 \mathrm{G}$ zero energy resonance. The solid curve of the upper panel indicates the bound state energy $E_{\mathrm{b}}(B)$ of the Feshbach molecule, while the dashed curve refers to the next more tightly bound dressed vibrational level. The dotted and dot-dashed lines are associated with the energies $E_{-1} / h=-24 \mathrm{MHz}$ of the bare highest excited vibrational level of $V_{\mathrm{bg}}(r)$ and $E_{\mathrm{res}}(B)$ of the closed channel resonance state, respectively. The crossing between the bare levels at $1001.7 \mathrm{G}$ leads to the measured variation of the magnetic moment of the Feshbach molecule indicated by the circles in the lower panel (Dürr et al., 2004a). For comparison, the solid and dashed curves refer to exact coupled channels calculations (van Kempen and Verhaar, 2004) and a two-channel approach (Góral et al., 2004), respectively.

Here $\sigma_{\mathrm{bg}}$ accounts for the range of the interaction, and $\langle\mathbf{r} \mid \mathbf{p}\rangle=$ $\exp (i \mathbf{p} \cdot \mathbf{r} / \hbar) /(2 \pi \hbar)^{3 / 2}$ denotes the plane wave associated with the relative momentum $\mathbf{p}$. Given this choice of form factor, the parameters $\sigma_{\text {bg }}$ and $\xi_{\text {bg }}$ are determined by the requirement that the bare Green's function of Eq. (72) exactly recovers $a_{\mathrm{bg}}$ and $E_{-1}$. The zero energy limit of the scattering amplitude associated with Eq. (72), i.e., $f_{\mathrm{bg}}(0)=-m \tau_{\mathrm{bg}}(0) /\left(4 \pi \hbar^{2}\right)$, yields the condition

$$
a_{\mathrm{bg}}=\sigma_{\mathrm{bg}} \frac{x}{1+x / \sqrt{\pi}}
$$

where $x=m \xi_{\mathrm{bg}} /\left(4 \pi \hbar^{2} \sigma_{\mathrm{bg}}\right)$ is a dimensionless variable. In addition, the bare energy level determined by the pole of the resonance term of Eq. (73) gives the following relation:

$$
1-\frac{x}{\sqrt{\pi}}\left[\sqrt{\pi} y e^{y^{2}} \operatorname{erfc}(y)-1\right]=0 .
$$

Here $\operatorname{erfc}(y)=\frac{2}{\sqrt{\pi}} \int_{y}^{\infty} e^{-u^{2}} d u$ is the complementary error function with the argument $y=\sqrt{m\left|E_{-1}\right|} \sigma_{\mathrm{bg}} / \hbar$.

For the example of the $1007 \mathrm{G}$ zero energy resonance of ${ }^{87} \mathrm{Rb}$ the energy $E_{-1}$ can be determined using the potential of Eq. (70). To this end, its parameter $r_{0}$ should be chosen in such a way that the number of vibrational levels is large compared to unity and that the known quantities $a_{\mathrm{bg}}$ and $C_{6}$ of Table IV are recovered. This yields $E_{-1} / h=$ $-24 \mathrm{MHz}$ in agreement with coupled channels calculations (van Kempen and Verhaar, 2004). Based on the precise values of $E_{-1}$ and $a_{\mathrm{bg}}$, Eqs. (77) and (78), in turn, give the range parameter and the amplitude of the separable potential to be $\sigma_{\text {bg }}=44 a_{\text {Bohr }}$ and $m \xi_{\text {bg }} /\left(4 \pi \hbar^{2}\right)=-339 a_{\text {Bohr }}$, respectively.

We note that the above adjustment of the effective entrance channel interaction is restricted to zero energy resonances with a positive background scattering length, i.e., $a_{\mathrm{bg}}>0$. In the opposite case, $a_{\mathrm{bg}}<0$, separable interactions do not support any bound state, similarly to the universal contact pseudo potential of Subsection III.F Among the experimentally relevant examples of Table IV only the broad, entrance-channel dominated zero energy resonances of ${ }^{6} \mathrm{Li}$ and ${ }^{85} \mathrm{Rb}$ have a negative background scattering length. Associated two-channel approaches describing the dressed energy levels beyond the Wigner threshold law regime exist, at least, in applications to the broad resonance of ${ }^{85} \mathrm{Rb}$ (Góral et al., 2005; Köhler et al., 2004; Kokkelmans and Holland, 2002). In the case of ${ }^{6} \mathrm{Li}$ a double resonance approach suitable for the description of many-body systems has been suggested (Kokkelmans et al., 2002).

Figures 12 and 13 reveal, however, that in both cases, ${ }^{85} \mathrm{Rb}$ and ${ }^{6} \mathrm{Li}$, the Feshbach molecule is well described by an effective single channel interaction, over a wide range of magnetic field strengths, in accordance with Subsection $\amalg I . F$ An appropriate magnetic field dependent single channel separable potential, suitable for applications to few-body bound states (Stoll and Köhler, 2005) as well as the dynamics of cold gases (Köhler et al., 2003a), may be constructed on the basis of Eqs. (74) and (76). The associated constant range parameter of the form factor is given by $\sigma_{\mathrm{bg}} \approx \sqrt{\pi} \bar{a} / 2$. The adjustment of $\xi_{\text {bg }}$ via Eq. (77) to the magnetic field dependent scattering length $a(B)$ of Eq. (39) instead of just $a_{\mathrm{bg}}$ ensures, in turn, that the near resonant energy $E_{\mathrm{b}}(B)$ of the Feshbach molecule recovers Eq. (66).

\section{The resonance energy}

In accordance with the single resonance approach of Eq. (36), the difference in energies of the resonance level and the entrance channel dissociation threshold, $E_{\text {res }}(B)$, characterises the closed channel part of the Hamiltonian. The associated relative magnetic moment, $\mu_{\text {res }}=\partial E_{\text {res }} / \partial B$, may be inferred from a Stern-Gerlach separation experiment, relying 
upon the force experienced by an atom due to the inhomogeneous magnetic field, B. Given any definite Zeeman state, this force is of the general form $\mathbf{F}_{\mathrm{a}}=-\boldsymbol{\nabla} E_{\mathrm{a}}$ where $E_{\mathrm{a}}$ is the Zeeman energy. The magnitude of $\mathbf{F}_{\mathrm{a}}=-\left(\partial E_{\mathrm{a}} / \partial B\right) \boldsymbol{\nabla}|\mathbf{B}|$ depends on the orientation quantum number $m_{f}$ of the total angular momentum with respect to the field direction. This dependence is well described by the Breit-Rabi formula (Breit and Rabi, 1931):

$$
\mathbf{F}_{\mathrm{a}}= \pm \frac{\frac{2 m_{f}}{2 i+1}+x}{2\left(1+\frac{4 m_{f}}{2 i+1} x+x^{2}\right)^{1 / 2}} g_{j} \mu_{\mathrm{Bohr}} \nabla|\mathbf{B}| .
$$

Here $i$ is the quantum number associated with the nuclear spin, $\mu_{\text {Bohr }}=9.27400949 \times 10^{-24} \mathrm{~J} / \mathrm{T}$ denotes the Bohr magneton, and the Lande factor, $g_{j} \approx 2$, refers to the electronic magnetic moment. The dimensionless variable, $x=g_{j} \mu_{\mathrm{Bohr}}|\mathbf{B}| / E_{\mathrm{hf}}$, depends on the field strength, $|\mathbf{B}|$, in addition to the hyperfine energy splitting in the absence of magnetic fields, $E_{\mathrm{hf}}$ (Arimondo et al., 1977). For any given orientation quantum number, $m_{f}$, and atomic species, the sign of the force of Eq. (79) is determined by the Zeeman multiplet.

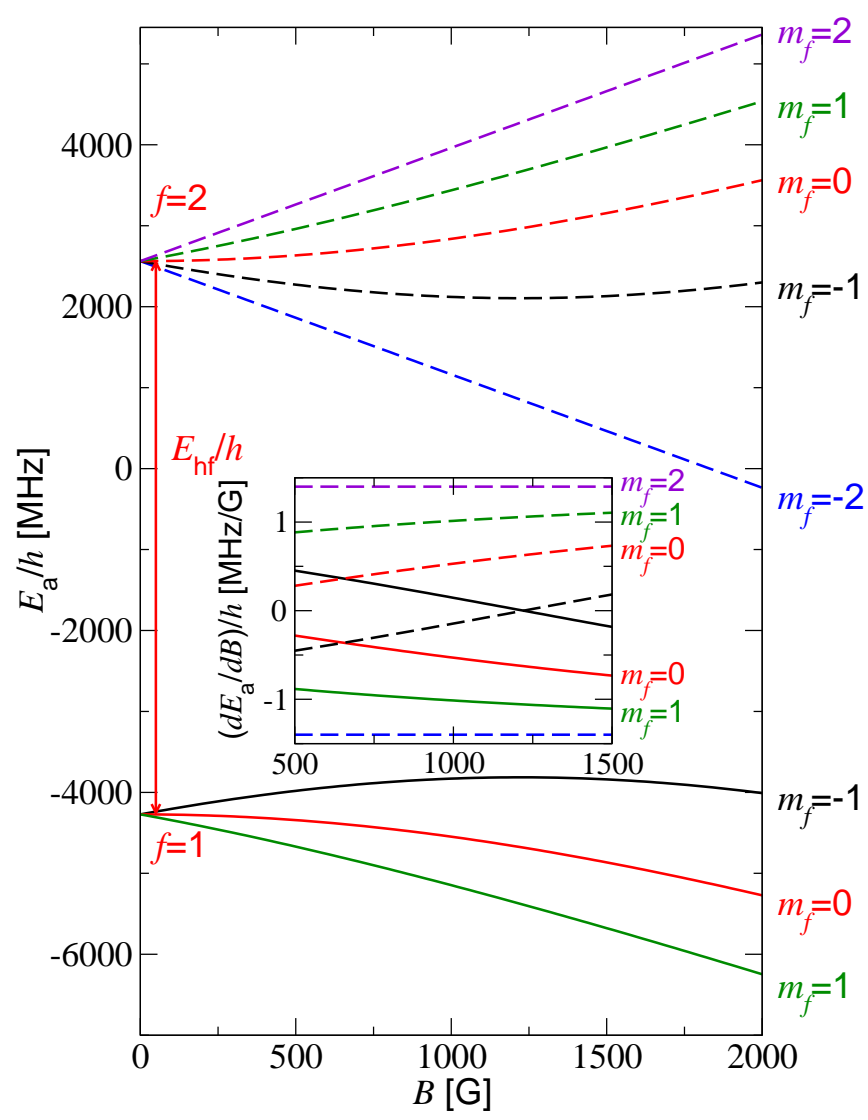

FIG. 17 (Colour in online edition) The Zeeman multiplets of ${ }^{87} \mathrm{Rb}$ associated with the total angular momentum quantum numbers $f=$ 1 (solid curves) and $f=2$ (dashed curves). The inset shows the magnetic moments, $\partial E_{\mathrm{a}} / \partial B$, determined by Eq. 79 and indicates the orientation quantum numbers $m_{f}$ of those Zeeman states that are relevant to Fig. 16 .

For the example illustrated in Fig. 17, the nuclear spin quantum number $i=3 / 2$ of ${ }^{87} \mathrm{Rb}$ (Arimondo et al., 1977) in addition to the electronic spin give rise to two Zeeman multiplets. These states adiabatically correlate with the hyperfine levels of total angular momentum quantum numbers $f=1$ and $f=2$ in the limit of zero magnetic field. The $s$-wave entrance channel spin configuration of any pair of the identical Bose atoms in the experiments of Fig. 16 is characterised by the quantum numbers $\left(f_{1}=1, m_{f_{1}}=+1 ; f_{2}=1, m_{f_{2}}=+1\right)$, referring to their electronic ground states. In accordance with Eq. (79) and the zero field hyperfine structure splitting of $E_{\mathrm{hf}} / h=6834 \mathrm{MHz}$ (Arimondo et al., 1977), the total magnetic moment associated with these ground state atoms at $B_{0}=1007.4 \mathrm{G}$ is determined by $2 \partial E_{\mathrm{a}} / \partial B=-h \times 2 \mathrm{MHz} / \mathrm{G}$. This derivative varies by less than $1 \%$ over the range of magnetic field strengths displayed in Fig. 16,

The predominant spin exchange interaction couples the pairs of ${ }^{87} \mathrm{Rb}$ ground state atoms to four $s$-wave scattering channels characterised by pairs of Zeeman levels, $\left(f_{1}, m_{f_{1}} ; f_{2}, m_{f_{2}}\right)$, whose total angular momentum orientation quantum number is conserved, i.e., $m_{f_{1}}+m_{f_{2}}=+2$. These closed channels are therefore described by $(1,1 ; 2,1)$, $(1,0 ; 2,2),(2,0 ; 2,2)$ and $(2,1 ; 2,1)$. In accordance with the inset of Fig. 17, their associated total magnetic moments at resonance amount to $0, h \times 0.9 \mathrm{MHz} / \mathrm{G}, h \times 1.9 \mathrm{MHz} / \mathrm{G}$, and $h \times 2 \mathrm{MHz} / \mathrm{G}$, respectively. The magnetic moment of the ${ }^{87} \mathrm{Rb}_{2}$ Feshbach molecule in the lower panel of Fig. 16 consists, in principle, of contributions from all five channels weighted by their admixtures. Both the experimental data (Dürr et al., 2004a) and the coupled channels predictions (van Kempen and Verhaar, 2004), however, are consistent with a Feshbach resonance state predominantly consisting of the pairs of Zeeman states just from the $f=2$ multiplet. The Breit-Rabi formula therefore yields an estimated difference in the magnetic moments of the resonance state and the entrance channel spin configuration of about $\mu_{\text {res }}=h \times 4 \mathrm{MHz} / \mathrm{G}$ in the vicinity of the zero energy resonance position. In an analogous manner the size of the parameter $\mu_{\text {res }}$ may be inferred from Eq. (79) for a variety of species. This procedure is particularly useful in the case of genuinely two-channel problems, such as, for instance, the spin configurations relevant to the $202 \mathrm{G}$ zero energy resonance of ${ }^{40} \mathrm{~K}$ (Bruun and Pethick, 2004). The recommended values of $\mu_{\text {res }}$ of Table $\nabla$ are all based on coupled channels predictions.

\section{Inter-channel coupling}

The inter-channel coupling leads to the decay width and energy shift of the bare resonance level due to its interaction with the background scattering continuum and entrance channel bare vibrational states. In accordance with Eq. (54), both quantities are determined by the parameters $\Delta B$ of Eq. (40) and $B_{0}-B_{\text {res }}$ of Eq. (41) in the Wigner threshold law domain. While the width in the magnetic field strength, $\Delta B$, is measured routinely, the associated shift is not directly observable. Its magnitude, however, may be inferred using general ideas developed in the context of multichannel quantum defect theory (Julienne and Mies, 1989; Mies and Raoult, 2000, 2004). 
TABLE V Parameters characterising the inter-channel coupling associated with several experimentally relevant zero energy resonances. Those values of $\Delta B$ and $\mu_{\text {res }}$ that are unreferenced refer to calculations performed for this review. In accordance with Eq. 69, the size of the parameter $\eta$ indicates whether a zero energy resonance is closed- or entrance-channel dominated.

\begin{tabular}{|c|c|c|c|c|}
\hline Species & $B_{0}[\mathrm{G}]$ & $\Delta B[\mathrm{G}]$ & $\mu_{\mathrm{res}} / h[\mathrm{MHz} / \mathrm{G}]$ & $\eta$ \\
\hline \multirow[t]{2}{*}{${ }^{6} \mathrm{Li}$} & 543.25(5) (Strecker et al., 2003) & 0.1 & 2.8 & 1215 \\
\hline & 834.149 (Bartenstein et al., 2005) & -300 (Bartenstein et al., 2005) & 2.8 & 0.02 \\
\hline \multirow[t]{2}{*}{${ }^{23} \mathrm{Na}$} & 853 (Stenger et al., 1999) & 0.01 (Mies et al., 2000) & 5.24 (Mies et al., 2000) & 1090 \\
\hline & 907 (Stenger et al., 1999) & 1.0 (Mies et al., 2000) & 5.24 (Mies et al., 2000) & 11 \\
\hline \multirow[t]{2}{*}{${ }^{40} \mathrm{~K}$} & 202.10(7) (Regal et al., 2004c) & 7.8(6) (Greiner et al., 2003) & 2.35 (Nygaard et al., 2006) & 0.46 \\
\hline & 224.21(5) (Regal and Jin, 2003) & 9.7(6) (Regal and Jin, 2003) & 2.35 (Nygaard et al., 2006) & 0.37 \\
\hline${ }^{85} \mathrm{Rb}$ & 155.0 (Thompson et al., 2005a) & 10.71(2) (Claussen et al., 2003) & -3.26 (Kokkelmans, 2002) & 0.04 \\
\hline${ }^{87} \mathrm{Rb}$ & 1007.40(4) (Volz et al., 2003) & 0.21 (Dürr et al., 2004b) & 4.2 (Dürr et al., 2004a) & 5.9 \\
\hline \multirow[t]{2}{*}{${ }^{133} \mathrm{Cs}$} & 19.90(3) (Chin et al., 2004b) & 0.005 (Julienne et al., 2004) & 0.798 (Julienne et al., 2004) & 437 \\
\hline & 47.97(3) (Chin et al., 2004b) & 0.15 (Julienne et al., 2004) & 2.09 (Julienne et al., 2004) & 0.99 \\
\hline
\end{tabular}

This yields the following approximate formula:

$$
B_{0}-B_{\mathrm{res}}=\Delta B \frac{a_{\mathrm{bg}}}{\bar{a}}\left[\frac{1-a_{\mathrm{bg}} / \bar{a}}{1+\left(1-a_{\mathrm{bg}} / \bar{a}\right)^{2}}\right] .
$$

Here $\bar{a}$ is the mean scattering length of Eq. (65). Consequently, the size of the resonance shift depends just on the quantities $a_{\mathrm{bg}}, \Delta B$, and $C_{6}$ which are all accessible to experimental studies. Entrance-channel dominated zero energy resonances, such as the example of ${ }^{85} \mathrm{Rb}$ illustrated in Fig. 12, tend to have large shifts comparable to the size of $\Delta B$. The predicted magnitude of $B_{0}-B_{\text {res }}=9 \mathrm{G}$ in Fig. 12 is consistent with the coupled channels binding energies (Kokkelmans, 2002). In the case of the closed-channel dominated $1007 \mathrm{G}$ zero energy resonance of ${ }^{87} \mathrm{Rb}$ in the upper panel of Fig. 16 the value of $B_{0}-B_{\text {res }}=0.07 \mathrm{G}$ of Eq. (65) is significantly smaller than the width. For all the examples given in Tables IV andV the corrections to Eq. (80) are negligible.

The quantity $W\left|\phi_{\text {res }}\right\rangle$, characterising the inter-channel coupling in the single resonance approach, should be adjusted in such a way that the matrix element of the bare Green's function in Eq. (54) recovers both $\Delta B$ and $B_{0}-B_{\text {res. }}$. Such an adjustment ensures, in particular, that the bound state energy of Eq. (47) properly interpolates between the universal and asymptotic regimes of magnetic field strengths. An explicit minimal implementation of the two-channel Hamiltonian of Eq. (16) may therefore be based, for instance, on the separable background scattering potential of Eq. (74) in addition to the following general expression:

$$
W\left|\phi_{\text {res }}\right\rangle=|\chi\rangle \zeta
$$

Here the amplitude $\zeta$ determines the overall magnitude of the inter-channel coupling and $\langle\chi\rangle$ its functional form. As the off diagonal potential, $W(r)$, is not resolved by the typically large de Broglie wave lengths associated with cold collisions, the form factor $\langle\chi\rangle$ may be chosen as a Gaussian function in momentum space, similarly to Eq. (76). This yields (Góral et al.,
2004):

$$
\langle\mathbf{p} \mid \chi\rangle=\chi(p)=\frac{\exp \left(-\frac{p^{2} \sigma^{2}}{2 \hbar^{2}}\right)}{(2 \pi \hbar)^{3 / 2}} .
$$

The associated range parameter, $\sigma$, and the amplitude, $\zeta$, are completely determined by the requirement that the imaginary and real parts of Eq. (54) recover the physical quantities $\Delta B$ and $B_{0}-B_{\text {res }}$, respectively. For the purpose of this adjustment, it is convenient to introduce the average range parameter $\bar{\sigma}=$ $\frac{1}{\sqrt{2}}\left(\sigma^{2}+\sigma_{\mathrm{bg}}^{2}\right)^{1 / 2}$. The imaginary part of the matrix element of the bare Green's function on the left hand side of Eq. (54) yields the first condition

$$
\Delta B=\frac{m|\zeta|^{2}}{4 \pi \hbar^{2} a_{\mathrm{bg}} \mu_{\mathrm{res}}}\left(1-\frac{a_{\mathrm{bg}}}{\sqrt{\pi} \bar{\sigma}}\right)^{2} .
$$

The associated widths of several experimentally relevant zero energy resonances are summarised in Table $\mathrm{V}$ Given the bare Green's function of Eq. (72) and Gaussian form factors, also the real part of Eq. (54) can be evaluated analytically. This leads to the second condition,

$$
B_{0}-B_{\mathrm{res}}=\Delta B \frac{a_{\mathrm{bg}}}{\sqrt{\pi} \sigma} \frac{1-\frac{a_{\mathrm{bg}}}{\sqrt{\pi} \sigma}\left(\frac{\sigma}{\bar{\sigma}}\right)^{2}}{\left(1-\frac{a_{\mathrm{bg}}}{\sqrt{\pi} \sigma} \frac{\sigma}{\bar{\sigma}}\right)^{2}},
$$

whose value for the resonance shift on the left hand side is provided by Eq. (80). Equations (83) and (84), in turn, simultaneously determine the parameters $\sigma$ and $\zeta$ characterising the inter-channel coupling. We note that the overall phase of the amplitude $\zeta$ is irrelevant to the physics described by the associated Hamiltonian.

For the example of the $1007 \mathrm{G}$ zero energy resonance of ${ }^{87} \mathrm{Rb}$ such an adjustment yields $\sigma=22 a_{\text {Bohr }}$ and $m|\zeta|^{2} /\left(4 \pi \hbar^{2} \sigma\right)=h \times 10 \mathrm{MHz}$. Given the effective background scattering potential of Eq. (74) and $\mu_{\text {res }}$ of TableV this procedure provides a complete implementation of the two-channel single resonance approach. Its predictions with respect to dressed binding energies are illustrated in the upper panel of 
Fig. 16 as well as in Fig. 12 The lower panel of Fig. 16 shows the magnetic moment of ${ }^{87} \mathrm{Rb}$ Feshbach molecules determined from the product $\mu_{\mathrm{res}} Z(B)$ via Eqs. (46) and (57) using the two-channel single resonance approach. The overall offset of the data of about $-2 \mathrm{MHz}$ refers to the magnetic moment associated with the entrance channel spin configuration. The comparisons with the measurements (Dürr et al., 2004a) and with the results of coupled channels calculations (van Kempen and Verhaar, 2004) indicate that the effective two-channel approach can fully recover the microscopic physics within the experimental energy range.

\section{ASSOCIATION OF FESHBACH MOLECULES}

The different experimental techniques for molecular association in cold gases all depend in one way or another on the properties of the diatomic energy spectra. Several approaches to the production of cold Feshbach molecules are based on the relaxation of an atomic gas into dimers near resonance (Jochim et al., 2003a; Zwierlein et al., 2003) or on dynamical sweeps of the magnetic field strength across $B_{0}$ (Cubizolles et al., 2003; Dürr et al., 2004a; Herbig et al., 2003; Regal et al., 2003a; Strecker et al., 2003; Xu et al., 2003). Both techniques take advantage of the degeneracy of the Feshbach molecular energy, $E_{\mathrm{b}}$, and the threshold for dissociation into free atoms in the limit $B \rightarrow B_{0}$. Relaxation of an atomic gas into dimer molecules requires collisions of at least three atoms to balance the energies and is commonly employed, to date, just in two spin component mixtures of ${ }^{6} \mathrm{Li}$ Fermi gases. The conceptually simpler molecular association via magnetic field sweeps seems to be more generally applicable to both Bose and Fermi gases and will therefore be the main subject of this section. Figure 16 illustrates its principle which relies, for the example of the $1007 \mathrm{G}$ zero energy resonance of ${ }^{87} \mathrm{Rb}$, upon the adiabatic transition from the diatomic zero energy continuum level to the bound state energy $E_{\mathrm{b}}(B)$ with decreasing $B$. Conversely, Fig. 12 suggests that in the case of the $155 \mathrm{G}$ zero energy resonance of ${ }^{85} \mathrm{Rb}$ free atom pairs may be associated to Feshbach molecules by increasing $B$ across $B_{0}$. The difference in energy of the colliding atoms and diatomic molecules is absorbed by the time dependent magnetic field. As, in general, the field is spatially homogeneous, the association process does not affect the centre of mass momentum of the atom pairs. From this viewpoint, the Feshbach molecules produced by magnetic field sweeps or other related dynamical techniques (Donley et al., 2002; Thompson et al., 2005a) are as cold as the atomic gas they originate from.

\section{A. Linear sweeps of the magnetic field strength}

In an idealised treatment of the molecular association the magnetic field strength may be assumed to vary linearly in time. This implies the relation

$$
B(t)=B_{\text {res }}+\dot{B}\left(t-t_{\text {res }}\right)
$$

where $\dot{B}$ is usually referred to as the ramp speed and $t_{\text {res }}$ is chosen to be the time at which the bare resonance energy, $E_{\text {res }}$, crosses the dissociation threshold of the entrance channel. The field strength, $B_{\text {res }}$, associated with $t_{\text {res }}$ is indicated in Fig. 12. In accordance with Eq. (35), the resonance energy is then also a linear function of time,

$$
E_{\text {res }}(t)=\dot{E}_{\text {res }}\left(t-t_{\text {res }}\right)
$$

with a constant derivative, $\dot{E}_{\text {res }}=\mu_{\text {res }} \dot{B}$. This formula presupposes that the magnetic field sweep is sufficiently slow for the electronic degrees of freedom of the atoms to adiabatically adjust to the changes in the magnetic field strength. To achieve molecular association of an atom pair, $\dot{E}_{\text {res }}$ needs to be negative (Mies et al., 2000). Consequently, the Feshbach resonance level is swept downward in time. This requirement determines $\dot{B}$ through the sign of $\mu_{\text {res }}$. Conversely, upward sweeps of the Feshbach resonance level across $B_{0}$ lead to heating of the atomic cloud. This general principle may be readily verified on the basis of the magnetic field dependence of the discrete spectrum of dressed energy levels of a trapped atom pair.

\section{Adiabatic association of Feshbach molecules}

Tight traps containing just a single pair of atoms may be realised, for instance, by the individual sites of an optical lattice (Jaksch et al., 2002; Tiesinga et al., 2000) or by microfabricated materials (Folman et al., 2002; Hinds et al., 2001; Long et al., 2003; Müller et al., 1999; Thywissen et al., 1999; Weinstein and Libbrecht, 1995). The two-body energy spectra associated with periodic potentials of optical lattices have been studied theoretically in the Wigner threshold law domain (Orso et al., 2005; Wouters and Orso, 2006) as well as observed experimentally (Moritz et al., 2005). While in the limit of high excitations the tunnelling of atoms is significant, the deepest localised diatomic levels of tight lattice sites are well described by the harmonic oscillator approximation. As a consequence, the centre of mass and relative motions of an atom pair confined to a single site can be treated separately, similarly to the two-body problem in free space.

A spherically symmetric harmonic confinement modifies the bare entrance channel Hamiltonian associated with the resonance enhanced interaction as follows:

$$
H_{\mathrm{bg}}=-\frac{\hbar^{2}}{m} \nabla^{2}+V_{\mathrm{bg}}(r)+V_{\text {trap }}(r) .
$$

Here $V_{\text {trap }}(r)$ denotes the potential energy of the isotropic harmonic oscillator in the barycentric frame which is given in terms of the reduced mass, $m / 2$, and the angular frequency, $\omega_{\text {ho }}$, to be:

$$
V_{\text {trap }}(r)=\frac{1}{2} \frac{m}{2} \omega_{\mathrm{ho}}^{2} r^{2} .
$$

Similarly to Eq. (25), the associated bare vibrational energy levels are determined by the stationary Schrödinger equation,

$$
H_{\mathrm{bg}} \phi_{v}(r)=E_{v} \phi_{v}(r) .
$$


Here the index $v=\ldots,-2,-1,0,1,2, \ldots$ labels the vibrational excitation in such a way that $v=0$ correlates adiabatically, in the limit $\omega_{\text {ho }} \rightarrow 0$, with the dissociation threshold of the entrance channel. According to this counting scheme, negative indices, $v<0$, are associated with the bare vibrational levels $E_{-1}, E_{-2}$, etc., indicated in Fig.10 In the limit of low vibrational excitation, $v \gtrsim 0$, the spatial extents of the bare states, including $\phi_{0}(r)$, are characterised by the trap length, $a_{\mathrm{ho}}=\sqrt{\hbar /\left(m \omega_{\mathrm{ho}}\right)}$. This length scale usually very much exceeds the modulus of the background scattering length, $\left|a_{\mathrm{bg}}\right|$. To first order in $a_{\mathrm{bg}} / a_{\mathrm{ho}}$ the energies of the excited levels are well approximated by the following formula (Busch et al., 1998):

$$
E_{v} \approx\left[\frac{3}{2}+2 v+\sqrt{\frac{2}{\pi}}\left(\begin{array}{c}
v+1 / 2 \\
v
\end{array}\right) \frac{a_{\mathrm{bg}}}{a_{\mathrm{ho}}}\right] \hbar \omega_{\mathrm{ho}} .
$$

Here $\left(\begin{array}{c}v+1 / 2 \\ v\end{array}\right)$ is a combinatorial. The dressed energy levels may be determined via the two-channel Hamiltonian of Eq. (16) using the single resonance approach of Eq. (36) (Mies et al., 2000), or via a single channel energy dependent contact interaction (Blume and Greene, 2002; Bolda et al., 2002). Both methods yield spectra consistent with full coupled channels calculations (Tiesinga et al., 2000).

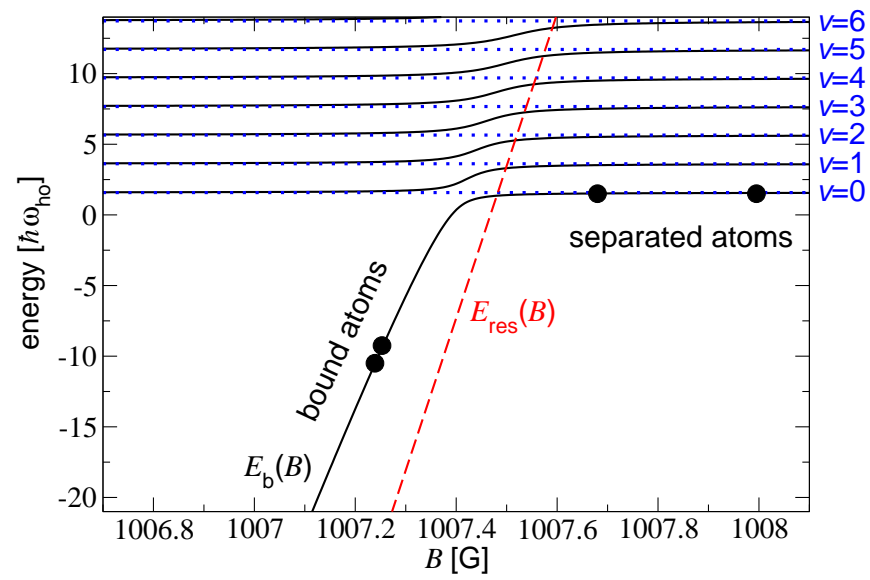

FIG. 18 (Colour in online edition) Schematic illustration of the molecular association of a pair of ground state ${ }^{87} \mathrm{Rb}$ atoms via a downward magnetic field sweep in a spherical harmonic atom trap with an oscillator frequency of $v_{\text {ho }}=39 \mathrm{kHz}$ (Thalhammer et al., 2006). The bare vibrational levels $(v=0, \ldots, 6)$ associated with the background scattering quasi continuum and the Feshbach resonance energy, $E_{\text {res }}(B)$, are indicated by dotted and dashed lines, respectively. Solid curves refer to the magnetic field dependence of the dressed energy levels in the vicinity of the zero energy resonance position of $B_{0}=1007.4 \mathrm{G}$.

Figure 18 shows the magnetic field dependence of the dressed two-body energy levels of ground state ${ }^{87} \mathrm{Rb}$ atoms predicted by the two-channel approach for a tight trap with a frequency of $v_{\mathrm{ho}}=\omega_{\mathrm{ho}} /(2 \pi)=39 \mathrm{kHz}$ (Thalhammer et al., 2006). An atom pair from a rubidium Bose-Einstein condensate prepared on the high field side of the $1007 \mathrm{G}$ zero energy resonance and loaded adiabatically into an optical lattice site is well described by the $v=0$ state. According to Fig. 18, this level adiabatically correlates with the Feshbach molecule on the low field side of $B_{0}$. Consequently, a magnetic downward sweep across $B_{0}$ associates the separated rubidium atoms to molecules with certainty in the limit of zero ramp speed, i.e. $\dot{B} \rightarrow 0$. An excited trap level with the vibrational quantum number $v$ is transferred to $v-1$. Conversely, an adiabatic upward magnetic field sweep across $B_{0}$ dissociates ${ }^{87} \mathrm{Rb}_{2}$ Feshbach molecules and leads to heating transitions from $v$ to $v+1$ in the excited trap levels. In general, the ramp direction for such cooling or heating transitions is determined just by the sign of $\mu_{\text {res }}$ or, equivalently, by the time variation of the resonance energy, $\dot{E}_{\text {res }}$.

\section{Exact time evolution of a single atom pair}

While the dressed energy levels of Fig. 18 reveal the mechanism of Feshbach molecular association in the limit of zero ramp speed, the dynamics of the diatomic wave function is described by the Schrödinger equation,

$$
i \hbar \frac{\partial}{\partial t}|\Psi(t)\rangle=H_{2 \mathrm{~B}}(t)|\Psi(t)\rangle .
$$

Here $H_{2 \mathrm{~B}}$ is the two-channel Hamiltonian of Eq. (16) in the single resonance approach of Eq. (36). Its time dependence is determined by the linear variation of the resonance energy of Eq. (86). The diatomic state, $|\Psi(t)\rangle$, has components in the entrance and closed channels, whose wave functions may be expanded into bare states, in accordance with the formulae

$$
\begin{aligned}
& \Psi_{\mathrm{bg}}(r, t)=\sum_{v} \phi_{v}(r) C_{v}(t), \\
& \Psi_{\mathrm{cl}}(r, t)=\phi_{\mathrm{res}}(r) C_{\mathrm{res}}(t) .
\end{aligned}
$$

Such a basis set expansion of Eq. (91) leads to the following dynamical equations for the associated time dependent coefficients

$$
\begin{aligned}
i \hbar \dot{C}_{v}(t) & =E_{v} C_{v}(t)+\left\langle\phi_{v}|W| \phi_{\text {res }}\right\rangle C_{\text {res }}(t), \\
i \hbar \dot{C}_{\text {res }}(t) & =E_{\text {res }}(t) C_{\text {res }}(t)+\sum_{v}\left\langle\phi_{\text {res }}|W| \phi_{v}\right\rangle C_{v}(t) .
\end{aligned}
$$

This configuration interaction approach is particularly useful for numerical treatments of Feshbach molecular association using any form of time dependent magnetic field variation (Mies et al., 2000). The special case of linear sweeps belongs to those quantum mechanical problems whose exact dynamics can be treated analytically (Demkov and Osherov, 1968; Macek and Cavagnero, 1998).

To this end, it is useful to split $H_{2 \mathrm{~B}}(t)$ into stationary and time dependent contributions:

$$
H_{2 \mathrm{~B}}(t)=H_{\text {stat }}+H_{\mathrm{cl}}(t) .
$$

Here the stationary Hamiltonian, $H_{\text {stat }}=H_{2 \mathrm{~B}}\left(t_{\text {res }}\right)$, is associated with the magnetic field strength $B_{\text {res }}$. In the single resonance approach the dynamical contribution, $H_{\mathrm{cl}}(t)$, is given by the following formula:

$$
H_{\mathrm{cl}}(t)=\left|\phi_{\mathrm{res}}, \mathrm{cl}\right\rangle E_{\mathrm{res}}(t)\left\langle\phi_{\mathrm{res}}, \mathrm{cl}\right| .
$$


Similarly to the effective potential of Eq. (74), the time dependent interaction, $H_{\mathrm{cl}}(t)$, involves only a single state associated with the closed channel, the product $\left|\phi_{\text {res }}, \mathrm{cl}\right\rangle=\left|\phi_{\text {res }}\right\rangle|\mathrm{cl}\rangle$. The following derivations will show that it is the separable form of Eq. (97) in addition to the linear dependence of the resonance energy $E_{\text {res }}$ on $t$ which allow for the analytic treatment of the time evolution.

The complete dynamics of an atom pair exposed to a linear magnetic field sweep may be inferred from the time evolution operator determined by the Schrödinger equation,

$$
i \hbar \frac{\partial}{\partial t} U_{2 \mathrm{~B}}\left(t, t^{\prime}\right)=H_{2 \mathrm{~B}}(t) U_{2 \mathrm{~B}}\left(t, t^{\prime}\right),
$$

in addition to the boundary condition $U_{2 \mathrm{~B}}(t, t)=1$. The diatomic state at time $t$ is thus given in terms of $U_{2 \mathrm{~B}}\left(t, t^{\prime}\right)$ and the state at time $t^{\prime}$ by

$$
|\Psi(t)\rangle=U_{2 \mathrm{~B}}\left(t, t^{\prime}\right)\left|\Psi\left(t^{\prime}\right)\right\rangle .
$$

Similarly, the dynamics in the absence of the interaction of Eq. (97) is described by $\exp \left[-i H_{\text {stat }}\left(t-t^{\prime}\right) / \hbar\right]$. Associated with these free and complete time evolution operators are the retarded Green's functions,

$$
\begin{aligned}
G_{\text {stat }}^{(+)}\left(t-t^{\prime}\right) & =\frac{1}{i \hbar} \theta\left(t-t^{\prime}\right) \exp \left[-i H_{\text {stat }}\left(t-t^{\prime}\right) / \hbar\right], \\
G_{2 \mathrm{~B}}^{(+)}\left(t, t^{\prime}\right) & =\frac{1}{i \hbar} \theta\left(t-t^{\prime}\right) U_{2 \mathrm{~B}}\left(t, t^{\prime}\right),
\end{aligned}
$$

where $\theta\left(t-t^{\prime}\right)$ is the step function which yields unity if $t>t^{\prime}$ and zero elsewhere. The Schrödinger equation (98) may be represented in terms of Eqs. (100) and (101) via the following formula:

$$
\begin{aligned}
G_{2 \mathrm{~B}}^{(+)}\left(t, t^{\prime}\right)= & G_{\mathrm{stat}}^{(+)}\left(t-t^{\prime}\right) \\
& +\int d \tau G_{\mathrm{stat}}^{(+)}(t-\tau) H_{\mathrm{cl}}(\tau) G_{2 \mathrm{~B}}^{(+)}\left(\tau, t^{\prime}\right) .
\end{aligned}
$$

Differentiation with respect to the variable $t$ readily verifies this relation by recovering the time derivative of the complete retarded Green's function of Eq. (101) which is directly determined by Eq. (98). The integral representation of Eq. (98) chosen in Eq. (102) is usually referred to as the post-form of the dynamical equation. The associated pre-form reads

$$
\begin{aligned}
G_{2 \mathrm{~B}}^{(+)}\left(t, t^{\prime}\right)= & G_{\mathrm{stat}}^{(+)}\left(t-t^{\prime}\right) \\
& +\int d \tau G_{2 \mathrm{~B}}^{(+)}(t, \tau) H_{\mathrm{cl}}(\tau) G_{\mathrm{stat}}^{(+)}\left(\tau-t^{\prime}\right)
\end{aligned}
$$

and can be verified similarly to the derivation of Eq. (102).

While, in general, the operator equation (102) requires a complete basis set expansion for its numerical solution, the separable form of Eq. (97) reduces this problem to the calculation of just the pair of matrix elements

$$
\begin{aligned}
g_{\text {stat }}^{(+)}\left(t-t^{\prime}\right) & =\left\langle\phi_{\text {res }}, \mathrm{cl}\left|G_{\text {stat }}^{(+)}\left(t-t^{\prime}\right)\right| \phi_{\text {res }}, \mathrm{cl}\right\rangle, \\
g_{2 \mathrm{~B}}^{(+)}\left(t, t^{\prime}\right) & =\left\langle\phi_{\text {res }}, \mathrm{cl}\left|G_{2 \mathrm{~B}}^{(+)}\left(t, t^{\prime}\right)\right| \phi_{\text {res }}, \mathrm{cl}\right\rangle .
\end{aligned}
$$

This follows from Eq. (97) via multiplying Eq. (102) by $\left\langle\phi_{\text {res }}, \mathrm{cl}\right|$ from the left and by $\left|\phi_{\text {res }}, \mathrm{cl}\right\rangle$ from the right which, in turn, determines Eq. 105) through the following integral equation:

$$
\begin{aligned}
g_{2 \mathrm{~B}}^{(+)}\left(t, t^{\prime}\right)= & g_{\text {stat }}^{(+)}\left(t-t^{\prime}\right) \\
& +\int d \tau g_{\text {stat }}^{(+)}(t-\tau) E_{\text {res }}(\tau) g_{2 \mathrm{~B}}^{(+)}\left(\tau, t^{\prime}\right) .
\end{aligned}
$$

Given the free retarded Green's function of Eq. (100), the complete time evolution operator can be inferred from the solution of Eq. (106) by inserting Eq. (103) into Eq. (102) and performing the time integrations.

Using the convolution theorem, a Fourier transform turns the integral on the right hand side of Eq. (106) into a product of functions. In the case of a linear magnetic field sweep such a procedure allows Eq. (106) to be solved analytically. To this end, it is instructive to introduce the energy dependent matrix elements,

$$
\begin{aligned}
g_{\text {stat }}(z) & =\int d t e^{i z\left(t-t^{\prime}\right) / \hbar} g_{\text {stat }}^{(+)}\left(t-t^{\prime}\right), \\
g_{2 \mathrm{~B}}\left(z, t^{\prime}\right) & =\int d t e^{i z\left(t-t^{\prime}\right) / \hbar} g_{2 \mathrm{~B}}^{(+)}\left(t, t^{\prime}\right),
\end{aligned}
$$

associated with the free and complete retarded Green's functions. Here the regularised argument " $z=E+i 0$ " ensures the convergence of the time integrals in the limit $t \rightarrow+\infty$ by approaching the real energy $E$ from the upper half of the complex plane. Given the resonance energy of Eq. 86 , a Fourier transform renders Eq. (106) into the following inhomogeneous first order linear differential equation:

$$
i \hbar \frac{\partial g_{2 \mathrm{~B}}\left(z, t^{\prime}\right)}{\partial E}=\hbar \varphi^{\prime}\left(z, t^{\prime}\right) g_{2 \mathrm{~B}}\left(z, t^{\prime}\right)+1 / \dot{E}_{\mathrm{res}} .
$$

Its dependence on the inter-atomic interaction is incorporated in the energy derivative $\varphi^{\prime}\left(z, t^{\prime}\right)=\partial \varphi\left(z, t^{\prime}\right) / \partial E$ of the complex phase

$$
\varphi\left(z, t^{\prime}\right)=-\frac{1}{\hbar \dot{E}_{\mathrm{res}}} \int_{0}^{E} \frac{d E^{\prime}}{g_{\mathrm{stat}}\left(z^{\prime}\right)}+E\left(t^{\prime}-t_{\mathrm{res}}\right) / \hbar,
$$

where " $z$ ' $=E^{\prime}+i 0$ " denotes a regularised integration variable with the same imaginary part as $z$.

The imaginary part of $\varphi\left(z, t^{\prime}\right)$ can be inferred from the Hamiltonian $H_{\text {stat }}$ using a spectral decomposition of $g_{\text {stat }}(z)$ analogous to Eq. (49). In particular, Eq. (53) determines the sign of $\operatorname{Im} \varphi\left(z, t^{\prime}\right)$ to be:

$$
\operatorname{sign}\left[\operatorname{Im} \varphi\left(z, t^{\prime}\right)\right]=-\operatorname{sign}\left(\dot{E}_{\text {res }}\right) \operatorname{sign}(\operatorname{Im} z) .
$$

Consequently, in the case of a downward ramp of the Feshbach resonance level, i.e. $\dot{E}_{\text {res }}<0$, the damped, retarded solution to Eq. (109) is given by

$$
g_{2 \mathrm{~B}}\left(z, t^{\prime}\right)=-\int_{E}^{\infty} d E^{\prime} \frac{e^{-i\left[\varphi\left(z, t^{\prime}\right)-\varphi\left(z^{\prime}, t^{\prime}\right)\right]}}{i \hbar \dot{E}_{\mathrm{res}}} .
$$

This formula may be verified from Eq. (109) by differentiation with respect to $E$. The matrix element of the complete retarded Green's function of Eq. (105) is just the inverse Fourier transform of Eq. (112) which yields:

$$
g_{2 \mathrm{~B}}^{(+)}\left(t, t^{\prime}\right)=-\int \frac{d E}{2 \pi \hbar} \int_{E}^{\infty} d E^{\prime} \frac{e^{-i\left[\varphi(z, t)-\varphi\left(z^{\prime}, t^{\prime}\right)\right]}}{i \hbar \dot{E}_{\mathrm{res}}} .
$$


The exact time evolution operator determined by Eq. (113) is applicable to the association of Feshbach molecules in free space as well as to the case of a trapped atom pair illustrated in Fig. 18 .

\section{Landau-Zener approach}

Already in 1932 Landau and Zener have independently derived the foundation for a simple estimate of the probability for molecule production in linear magnetic field sweeps (Landau, 1932; Zener, 1932). Their generic approaches may be interpreted in terms of a coupled system of two channels, each of which containing just a single state. In applications to Feshbach molecular association such a treatment is equivalent to the single resonance approach of Eq. (36) in addition to the following replacement of the entrance channel Hamiltonian:

$$
H_{\text {bg }} \rightarrow\left|\phi_{0}\right\rangle E_{0}\left\langle\phi_{0}\right|
$$

Here $\left|\phi_{0}\right\rangle$ may be interpreted, for instance, in terms of the zeroth vibrational state of the relative motion of a trapped atom pair with the energy $E_{0}$. This reduction of the twochannel continuum to a two-level system gives rise to analytic solutions of the coupled set of stationary Schrödinger equations (20) and (21). Given the simple form of the entrance channel Green's function in the Landau-Zener approach, i.e.

$$
G_{\mathrm{bg}}(z)=\left|\phi_{0}\right\rangle \frac{1}{z-E_{0}}\left\langle\phi_{0}\right|
$$

Eq. (47) determines the two-level dressed energies to be:

$$
E_{ \pm}=\frac{E_{0}+E_{\mathrm{res}}}{2} \pm \frac{\left|E_{0}-E_{\mathrm{res}}\right|}{2} \sqrt{1+4 \frac{\left|\left\langle\phi_{\mathrm{res}}|W| \phi_{0}\right\rangle\right|^{2}}{\left(E_{0}-E_{\mathrm{res}}\right)^{2}}} .
$$

The magnetic field dependent slopes of the levels $E_{+}$and

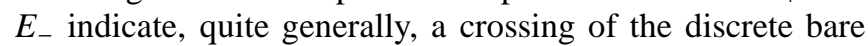
entrance- and closed-channel energies, $E_{0}$ and $E_{\text {res }}$, respectively. Analytic representations of the associated dressed twocomponent stationary states, $\left|\phi_{+}\right\rangle$and $\left|\phi_{-}\right\rangle$, can be inferred from Eq. 45.

In accordance with Eq. (116), a downward sweep of $E_{\text {res }}$ across $E_{0}$ transfers a pair of atoms in the initial state $\left|\phi_{0}, \mathrm{bg}\right\rangle$ into the final state $\left|\phi_{\text {res }}, \mathrm{cl}\right\rangle$ in the limit of zero ramp speed. Their energies adiabatically follow the $E_{-}$curve. Given the linear variation of $E_{\text {res }}$ of Eq. (86), such a simplified scenario presupposes the sweep to start and end asymptotically far from the crossing point of the bare levels. This, in turn, implies the formal limits $t_{\mathrm{i}} \rightarrow-\infty$ and $t_{\mathrm{f}} \rightarrow \infty$ of the initial and final times, respectively. The adiabatic energy variation of the Landau-Zener two-level approach is similar to the harmonic trap case illustrated in Fig. 18, except that the quasi continuum of excited levels with the indices $v>0$ is neglected.

Finite ramp speeds allow an atom pair to end up in a superposition of the entrance and closed channel bare states $\left|\phi_{0}, \mathrm{bg}\right\rangle$ and $\left|\phi_{\text {res }}, \mathrm{cl}\right\rangle$, respectively. In accordance with Eq. (105) and the assumption of a two-level system, the probability for an asymptotic transition between the bare levels is given by

$$
\begin{aligned}
p_{0, \text { res }} & =\left|\left\langle\phi_{\text {res }}, \mathrm{cl}\left|U_{2 \mathrm{~B}}\left(t_{\mathrm{f}}, t_{\mathrm{i}}\right)\right| \phi_{0}, \mathrm{bg}\right\rangle\right|^{2} \\
& =1-\left|i \hbar g_{2 \mathrm{~B}}\left(t_{\mathrm{f}}, t_{\mathrm{i}}\right)\right|^{2}
\end{aligned}
$$

in the limits $t_{\mathrm{i}} \rightarrow-\infty$ and $t_{\mathrm{f}} \rightarrow \infty$. These time limits can be determined analytically using the stationary phase condition for the energy integrals over the oscillatory functions on the right hand side of Eq. 113). This exact approach relies upon the observation that asymptotically only those regions close to zeros of the derivatives of the phases $\varphi^{\prime}\left(z, t_{\mathrm{i}}\right)$ and $\varphi^{\prime}\left(z, t_{\mathrm{f}}\right)$ contribute to the integrals. All the remaining energy ranges in which the complex exponentials are rapidly oscillating yield negligible averages.

The positions of the stationary phases can be readily found in the case of a two-level system. An explicit determination of the stationary Green's function, $G_{\text {stat }}(z)=\left(z-H_{\text {stat }}\right)^{-1}$, associated with the Hamiltonian $H_{\text {stat }}$ of Eq. 96 yields

$$
1 / g_{\text {stat }}(z)=z-\left\langle\phi_{\text {res }}\left|W G_{\text {bg }}(z) W\right| \phi_{\text {res }}\right\rangle \text {. }
$$

This formula and Eq. (110) give the derivative of the phase to be

$$
\varphi^{\prime}(z, t)=-\frac{E-E_{\mathrm{res}}(t)-\left\langle\phi_{\mathrm{res}}\left|W G_{\mathrm{bg}}(z) W\right| \phi_{\mathrm{res}}\right\rangle}{\hbar \dot{E}_{\mathrm{res}}} .
$$

Consequently, $\varphi^{\prime}(z, t)$ vanishes at the dressed energies $E_{ \pm}$associated with the magnetic field strength at time $t$, in accordance with Eq. (47).

As only those regions of parameters $E$ and $E^{\prime}$ in the close proximity of $E_{ \pm}$significantly contribute to Eq. (113), the phases may be expanded to second order about their stationary points. This yields:

$$
\varphi(z, t) \approx \varphi\left(z_{ \pm}, t\right)+\frac{1}{2} \varphi^{\prime \prime}\left(E_{ \pm}, t\right)\left(E-E_{ \pm}\right)^{2}
$$

Here $z_{ \pm}=E_{ \pm}+i 0$ denotes the regularised energy parameter associated with $E_{ \pm}$at time $t$. The second derivative,

$$
\varphi^{\prime \prime}\left(E_{ \pm}, t\right)=-\frac{1}{\hbar \dot{E}_{\mathrm{res}}}\left[1+\left\langle\phi_{\mathrm{res}}\left|W G_{\mathrm{bg}}^{2}\left(E_{ \pm}\right) W\right| \phi_{\mathrm{res}}\right\rangle\right],
$$

is always positive in the case of a downward ramp of $E_{\text {res }}(t)$ and its inverse plays the role of a variance in the complex Gaussian integrals over $E$ and $E^{\prime}$. Performing the Gaussian integration associated with $t_{\mathrm{i}}$ on the right hand side of Eq. (113) gives

$$
\int_{E}^{\infty} d E^{\prime} e^{i \varphi\left(z^{\prime}, t_{\mathrm{i}}\right)} \approx \sqrt{2 \pi} \sum_{n= \pm} \frac{e^{i\left[\varphi\left(z_{n}^{\mathrm{i}}, t_{\mathrm{i}}\right)+\pi / 4\right]}}{\sqrt{\varphi^{\prime \prime}\left(E_{n}^{\mathrm{i}}, t_{\mathrm{i}}\right)}} \theta\left(E_{n}^{\mathrm{i}}-E\right) .
$$

Here $z_{ \pm}^{\mathrm{i}}$ and $E_{ \pm}^{\mathrm{i}}$ refer to the dressed energies at the initial magnetic field strength. Their asymptotic values in the case of a downward sweep of $E_{\text {res }}$ are given by $E_{+}^{\mathrm{i}} \rightarrow \infty$ and $E_{-}^{\mathrm{i}} \rightarrow E_{0}$ in the limit $t_{\mathrm{i}} \rightarrow-\infty$. This implies $\varphi^{\prime \prime}\left(E_{+}^{\mathrm{i}}, t_{\mathrm{i}}\right) \rightarrow-1 /\left(\hbar \dot{E}_{\mathrm{res}}\right)$ and $\varphi^{\prime \prime}\left(E_{-}^{\mathrm{i}}, t_{\mathrm{i}}\right) \rightarrow \infty$. Consequently, only the term associated with $E_{+}^{\mathrm{i}}$ significantly contributes to the sum of Eq. (122). 
The integral over the parameter $E$ on the right hand side of Eq. (113) can be evaluated similarly to Eq. (122). This yields

$$
i \hbar g_{2 \mathrm{~B}}^{(+)}\left(t_{\mathrm{f}}, t_{\mathrm{i}}\right)=e^{-i\left[\varphi\left(E_{-}^{\mathrm{f}}, t_{\mathrm{f}}\right)-\varphi\left(E_{+}^{\mathrm{i}}, t_{\mathrm{i}}\right)\right]}
$$

in the limits $t_{\mathrm{i}} \rightarrow-\infty$ and $t_{\mathrm{f}} \rightarrow \infty$. Here $E_{-}^{\mathrm{f}}$ refers to the dressed energy with the asymptotic behaviour $E_{-} \rightarrow-\infty$ at the final time of the magnetic field sweep. The imaginary parts of the phases $\varphi\left(E_{-}^{\mathrm{f}}, t_{\mathrm{f}}\right)$ and $\varphi\left(E_{+}^{\mathrm{i}}, t_{\mathrm{i}}\right)$ relevant to the transition probability of Eq. (117) may be obtained from Eqs. (110) and (53). This leads to the Landau-Zener formulae,

$$
\begin{aligned}
p_{0, \mathrm{res}} & =1-e^{-2 \pi \delta_{\mathrm{LZ}}}, \\
p_{0,0} & =e^{-2 \pi \delta_{\mathrm{LZ}}} .
\end{aligned}
$$

Here $p_{0,0}$ denotes the probability for detecting the atom pair in the bare state $\left|\phi_{0}, b g\right\rangle$ at the end of the magnetic field sweep, and

$$
\delta_{\mathrm{LZ}}=\frac{\left|\left\langle\phi_{\mathrm{res}}|W| \phi_{0}\right\rangle\right|^{2}}{\hbar\left|\dot{E}_{\mathrm{res}}\right|}
$$

is the Landau-Zener parameter.

For a pair of atoms in an isotropic harmonic trap the matrix element involving the inter-channel coupling $W$ can be inferred from Eq. (40) via the general approximate relation

$$
\left|\left\langle\phi_{\text {res }}|W| \phi_{v}\right\rangle\right|^{2} \approx 2 \pi m \sqrt{m E_{v}}\left|\left\langle\phi_{\text {res }}|W| \phi_{0}^{(+)}\right\rangle\right|^{2} \frac{\partial E_{v}}{\partial v} .
$$

Here $E_{v}$ is the energy of the bare oscillator level of Eq. 90. in the limit of low excitations in the Wigner threshold law regime. Under the assumption that the single particle trap length very much exceeds the background scattering length, i.e. $\left|a_{\mathrm{bg}}\right| / a_{\mathrm{ho}} \ll 1$, the density of states is given by $\partial E_{v} / \partial v=$ $2 \hbar \omega_{\text {ho }}$. Consequently, Eqs. (40) and (127) determine the Landau-Zener parameter associated with a trapped atom pair to be (Julienne et al., 2004):

$$
\delta_{\mathrm{LZ}}^{\mathrm{ho}}=\frac{\sqrt{6} \hbar}{\pi m a_{\mathrm{ho}}^{3}}\left|\frac{a_{\mathrm{bg}} \Delta B}{\dot{B}}\right| .
$$

Figure 19 illustrates the validity of the Landau-Zener approach in applications to Feshbach molecular association and dissociation in an optical lattice where the sites are filled with two atoms (Thalhammer et al., 2006).

On the basis of Eq. (113), the preceding determination of the asymptotic behaviour of transition amplitudes may be extended to an arbitrary number of energy states of the entrance channel interaction (Demkov and Osherov, 1968; Macek and Cavagnero, 1998; Yurovsky and Ben-Reuven, 1998; Yurovsky et al., 1999a). This shows that despite the fact that the Landau-Zener approach neglects the bare excited and molecular levels, it gives the exact probability for the loss of atom pairs from the $v=0$ mode of a harmonic trap. At the end of an asymptotic linear downward sweep of $E_{\text {res }}$ all population either remains in the initial $v=0$ state or is transferred either into energetically lower levels, $v<0$, or into the resonance state. A similar statement applies in the

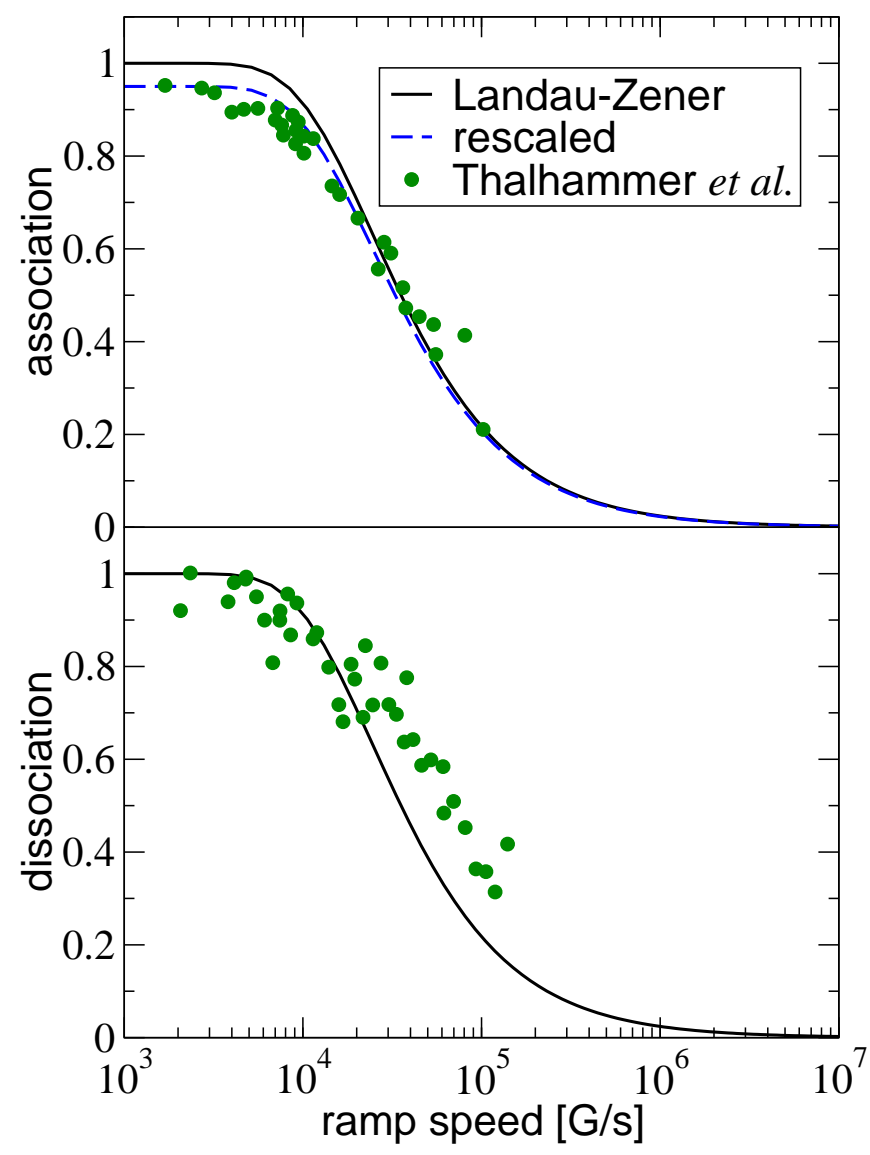

FIG. 19 (Colour in online edition) Efficiency of Feshbach molecular association (upper panel) and dissociation into the lowest energy band of an optical lattice (lower panel) via linear magnetic field sweeps across the $1007 \mathrm{G}$ zero energy resonance of ${ }^{87} \mathrm{Rb}$ versus the ramp speed (Thalhammer et al., 2006). The solid curves refer to the Landau-Zener formula of Eq. (124) and the coefficient for a harmonic oscillator of Eq. (128) using the measured frequency of $v_{\text {ho }}=39 \mathrm{kHz}$. The dashed curve indicates the same Landau-Zener prediction scaled by a factor of 0.95 which accounts for possible deficits in the ideal diatomic filling, for instance, due to tunnelling between lattice sites (Thalhammer et al., 2006).

case of asymptotic upward sweeps which are also exactly described by Eqs. (124), (125) and (128). Consequently, in the limits $t_{\mathrm{i}} \rightarrow-\infty$ and $t_{\mathrm{f}} \rightarrow \infty$ transitions between states occur only in the intuitive direction of the sweep. The intermediate dynamics, however, involves all levels, i.e., the amplitudes associated with unintuitive transitions interfere away only at asymptotically large times.

\section{B. Magnetic field sweeps in Bose-Einstein condensates}

Several pioneering studies on the properties of zero energy resonances of alkali atom pairs (Cornish et al., 2000; Inouye et al., 1998; Stenger et al., 1999) have been performed in dilute Bose-Einstein condensates (Anderson et al., 1995; Bradley et al., 1997, 1995; Davis et al., 1995). Condensation of Bose atoms (Bose, 1924; Einstein, 1924, 1925) occurs 
when the occupation number $N_{\mathrm{c}}$ of a particular single particle state becomes comparable to the total number of atoms, $N$, in such a way that $N_{\mathrm{c}} / N$ remains finite in the thermodynamic limit. The signatures of this phenomenon in dilute alkali gases have been identified, for instance, through a specific, narrow momentum distribution of the atoms or a characteristic spectrum of collective excitations (Dalfovo et al., 1999). While low collision momenta facilitate the theoretical description of the two-body physics of molecular association, the complex many-particle nature of Bose-Einstein condensates subject to dynamically resonance enhanced interactions becomes particularly significant on long time scales. In addition, the Feshbach molecules of some species of alkalis associated with closed-channel dominated resonances proved to be unstable in the environment of a gas (Dürr et al., 2004a; Herbig et al., 2003; Xu et al., 2003). For this reason, such experiments were performed, in part, under conditions of free expansion (Dürr et al., 2004a; Mark et al., 2005; Yurovsky and Ben-Reuven, 2004, 2005). Comparisons between predicted and measured molecule production via magnetic field sweeps in Bose-Einstein condensates are, therefore, less conclusive than in the case of a single atom pair confined to an optical lattice site.

\section{Limit of high ramp speeds}

In the limit of high ramp speeds the transfer of condensed atoms into Feshbach molecules may be estimated using the two-body Landau-Zener approach (Góral et al., 2004; Mies et al., 2000). To this end, it is instructive to divide the dilute gas into regions of virtually constant density. Their volumes, $\mathcal{V}$, can be chosen sufficiently large for the thermodynamic limit to be applicable. The state of an arbitrary atom pair of a uniform Bose-Einstein condensate in each of these periodic boxes is well described by the lowest quasi continuum energy level. Accordingly, the associated Landau-Zener parameter can be inferred from Eq. (126) using the following replacement of the initial wave function:

$$
\left|\phi_{0}\right\rangle \rightarrow\left|\phi_{0}^{(+)}\right\rangle \sqrt{(2 \pi \hbar)^{3} / \mathcal{V}}
$$

Given that the size of a dilute gas is on the order of several $\mu \mathrm{m}$, the volume $\mathcal{V}$ is sufficiently large for the transition probability of Eq. 124 to reduce just to its first order approximation, $p_{0 \text {,res }} \approx 2 \pi \delta_{\mathrm{LZ}}$. A typical order of magnitude of $p_{0, \text { res }}$ is $10^{-6}$ (Mies et al., 2000).

While the association of a particular pair of condensed atoms is a rare event, the fact that each atom has all the others to interact with grossly enhances the efficiency of molecule formation. Just in the limit of high ramp speeds the BoseEinstein condensate may be treated as a reservoir whose total atom number is hardly affected by an asymptotic magnetic field sweep across $B_{0}$. This assumption implies that the small fraction of lost atoms is well described in terms of the pairwise average of the microscopic transition probabilities. The number of pairs in a box with $N$ atoms is $N(N-1) / 2$ which in the limit $|\dot{B}| \rightarrow \infty$ yields the following estimate for the condensate depletion (Góral et al., 2004):

$$
N_{\text {loss }}=2 \pi(N-1)(N / \mathcal{V}) \frac{4 \pi \hbar}{m}\left|\frac{a_{\mathrm{bg}} \Delta B}{\dot{B}}\right| .
$$

Here $N / \mathcal{V}$ is the uniform density of the gas in the volume $\mathcal{V}$. In accordance with the local density approximation, the total number of atoms lost from a Bose-Einstein condensate is given by the spatial average over the densities of all boxes.

We note that the Landau-Zener estimate of Eq. 130 is applicable to both sweep directions of the Feshbach resonance level. In the case of downward sweeps the condensed atoms are partly transferred into diatomic Feshbach molecules whose final number, $N_{\mathrm{d}}^{\mathrm{f}}$, equals one half of the atom loss of Eq. (130), i.e.,

$$
N_{\mathrm{d}}^{\mathrm{f}}=N_{\mathrm{loss}} / 2
$$

Upward sweeps lead to the production of correlated pairs with a comparatively high relative velocity depending on the ramp speed. In some experiments using nonlinear field variations (Donley et al., 2001, 2002) such atom pairs were detected as a trapped dilute cloud with an average spatial extent much larger than the size of the remnant Bose-Einstein condensate.

\section{Two-level mean field approach}

A description of atom loss from a Bose-Einstein condensate consistent with both Eq. (130) and the dynamical depletion of pairs during a magnetic field sweep across $B_{0}$ may be based on Eqs. (94) and (95). Such an extended two-level configuration interaction approach to the probability amplitudes, $C_{0}(t)$ and $C_{\text {res }}(t)$, associated with the zero energy mode of an atom pair and its depletion, respectively, is given by the formulae (Góral et al., 2004; Julienne et al., 2004):

$$
\begin{aligned}
i \hbar \dot{C}_{0}(t) & =E_{0} C_{0}(t)+(N / \mathcal{V})^{1 / 2} g_{\text {res }}^{*} C_{0}^{*}(t) C_{\text {res }}(t), \\
i \hbar \dot{C}_{\text {res }}(t) & =E_{\text {res }}(t) C_{\text {res }}(t)+(N / \mathcal{V})^{1 / 2} g_{\text {res }} C_{0}^{2}(t)
\end{aligned}
$$

Here $N$ is the total number of atoms of the homogeneous gas in the volume $\mathcal{V}$. The inter-channel coupling is determined by the matrix element

$$
g_{\text {res }}=(2 \pi \hbar)^{3 / 2}\left\langle\phi_{\text {res }}|W| \phi_{0}^{(+)}\right\rangle,
$$

in accordance with Eqs. (94), (95) and (129). Similarly to the two-body configuration interaction approach, Eqs. (132) and (133) lead to a constant of motion, $\left|C_{0}(t)\right|^{2}+\left|C_{\text {res }}(t)\right|^{2}=1$. This implies an interpretation of the quantities $N\left|C_{0}(t)\right|^{2}$ and $N\left|C_{\text {res }}(t)\right|^{2}$ in terms of the numbers of atoms associated with the remnant Bose-Einstein condensate and correlated pairs, respectively. The nonlinear nature of Eqs. (132) and (133) in terms of the Bose enhancement factor, $(N / \mathcal{V})^{1 / 2} C_{0}(t)$, ensures consistency with Eq. (130) and, therefore, accounts for the surrounding gas.

The long time asymptotic populations may be estimated analytically based on a linearised version of Eqs. (132) and (133) using a static Bose enhancement factor, $(N / \mathcal{V})^{1 / 2}($ Mies et al. 
2000). This treatment of the inter-channel coupling neglects the time dependence of the depletion of pairs of condensed atoms and leads to dynamical equations formally equivalent to those of the two-body Landau-Zener approach. In accordance with Eq. (130), the associated Landau-Zener coefficient is given by

$$
\delta_{\mathrm{LZ}}^{\mathrm{BEC}}=(N / \mathcal{V}) \frac{4 \pi \hbar}{m}\left|\frac{a_{\mathrm{bg}} \Delta B}{\dot{B}}\right|=N \delta_{\mathrm{LZ}} .
$$

The asymptotic condensate depletion and its remnant uniform density can be inferred from Eqs. (124) and (125), respectively, using $\delta_{\mathrm{LZ}}^{\mathrm{BEC}}$ instead of $\delta_{\mathrm{Lz}}$.

An approach similar to Eqs. (132) and (133) but applicable to trapped gases beyond a local density treatment was derived on the basis of a mean field approximation (Drummond et al., 1998; Timmermans et al., 1998, 1999a; Tommasini et al., 1998). The associated many-body model Hamiltonian was originally introduced in the context of superconductivity (Friedberg and Lee, 1989; Ranninger and Robaszkiewicz, 1985). This procedure leads to the following dynamical equations:

$$
\begin{aligned}
i \hbar \dot{\Psi}(\mathbf{x}, t) & =H_{\mathrm{GP}} \Psi(\mathbf{x}, t)+g_{\mathrm{res}}^{*} \Psi^{*}(\mathbf{x}, t) \Psi_{\text {res }}(\mathbf{x}, t), \\
i \hbar \dot{\Psi}_{\mathrm{res}}(\mathbf{R}, t) & =H_{\mathrm{res}}(t) \Psi_{\mathrm{res}}(\mathbf{R}, t)+g_{\mathrm{res}} \Psi^{2}(\mathbf{R}, t) .
\end{aligned}
$$

Here the background scattering is included in terms of the usual Gross-Pitaevskii mean field Hamiltonian, $H_{\mathrm{GP}}$, in the contact pseudo interaction approximation (Gross, 1961; Pitaevskii, 1961). Given a spherically symmetric harmonic atom trap, $H_{\mathrm{GP}}$ therefore consists of the following contributions:

$$
H_{\mathrm{GP}}=-\frac{\hbar^{2} \nabla^{2}}{2 m}+\frac{m}{2} \omega_{\mathrm{ho}}^{2}|\mathbf{x}|^{2}+\frac{4 \pi \hbar^{2}}{m} a_{\mathrm{bg}}|\Psi(\mathbf{x}, t)|^{2} .
$$

Typical frequencies $v_{\text {ho }}=\omega_{\text {ho }} /(2 \pi)$ associated with such comparatively weakly confining traps are on the order of $100 \mathrm{~Hz}$. The generalised resonance energy, $H_{\text {res }}(t)$, contains the centre of mass kinetic energy of correlated pairs as well as a magnetic field shift from $B_{\text {res }}$ to the measurable position of the singularity of the scattering length. Its explicit expression reads:

$$
H_{\mathrm{res}}(t)=-\frac{\hbar^{2} \nabla^{2}}{4 m}+m \omega_{\mathrm{ho}}^{2}|\mathbf{R}|^{2}+\mu_{\mathrm{res}}\left[B(t)-B_{0}\right]
$$

The mean fields $\Psi(\mathbf{x}, t)$ and $\Psi_{\text {res }}(\mathbf{R}, t)$ refer to the amplitudes of the densities of atoms in the condensate at the position $\mathbf{x}$ and of correlated pairs with the centre of mass $\mathbf{R}$, respectively. Similarly to the configuration interaction approach, Eqs. (136) and (137) give rise to a constant of motion consistent with the conservation of the total number of atoms:

$$
\int d \mathbf{x}|\Psi(\mathbf{x}, t)|^{2}+\int d \mathbf{R}\left|\Psi_{\text {res }}(\mathbf{R}, t)\right|^{2}=N .
$$

Since its first applications in the context of Feshbach resonances in the physics of cold gases, the concept of this two-level mean field approach has been continually extended to a variety of physical situations (van Abeelen and Verhaar,
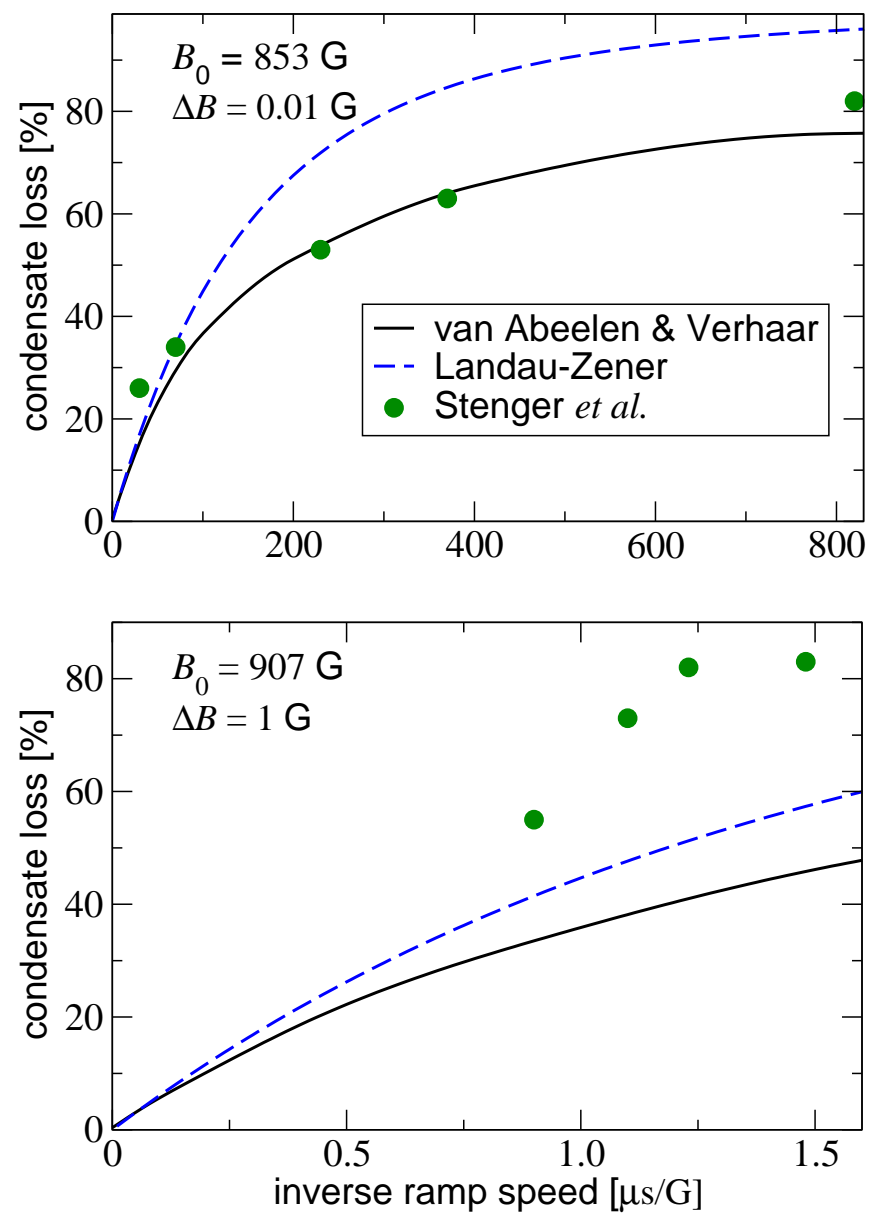

FIG. 20 (Colour in online edition) Loss of condensate atoms in upward sweeps of the Feshbach resonance level across the $853 \mathrm{G}$ and $907 \mathrm{G}$ zero energy resonances of ${ }^{23} \mathrm{Na}$ versus the inverse ramp speed, $1 /|\dot{B}|$ (Stenger et al., 1999). The experimental data are compared to theoretical predictions using the LandauZener (Góral et al., 2004; Mies et al., 2000) and two-level mean field (van Abeelen and Verhaar, 1999) approaches. The theoretical Feshbach resonance parameters employed refer to the values of $a_{\mathrm{bg}}$ and $\Delta B$ given in Tables $[\mathrm{IV}$ and $\mathrm{V}$ We note the differences in the ramp speeds of three orders of magnitude between the upper and lower panels which reflect the different widths of the $853 \mathrm{G}$ and $907 \mathrm{G}$ zero energy resonances.

1999; Duine and Stoof, 2003b; Góral et al., 2001; Holland et al., 2001; Yurovsky and Ben-Reuven, 2003b).

Figure 20 shows that Eqs. (136) and (137) give reasonable agreement with the loss of condensed atoms observed in experiments involving zero energy resonances of ${ }^{23} \mathrm{Na}$ (Stenger et al., 1999). These measurements refer to asymptotic upward sweeps of the Feshbach resonance level across $B_{0}$ leading to the production of unbound correlated pairs of atoms (van Abeelen and Verhaar, 1999). We note that Eqs. (136) and (137) well account for the onset of atom loss over ranges of ramp speeds which differ by three orders of magnitude between the upper and lower panels of Fig.20. A similarly fair agreement has been reported for predictions of several different approaches (Köhler et al., 2004; 
Mackie et al., 2002a; Yurovsky and Ben-Reuven, 2003a) on the observed atom loss of a Bose-Einstein condensate exposed to magnetic field sweeps across the $155 \mathrm{G}$ zero energy resonance of ${ }^{85} \mathrm{Rb}$ (Cornish et al., 2000). The dashed curves in Fig. 20 indicate the asymptotic Landau-Zener predictions based on the coefficient of Eq. (135) and the local density approximation. Both theoretical approaches recover the asymptotic behaviour of the loss of condensed atoms in the case of fast sweeps given by Eq. (130). Their functional forms differ in the opposite, adiabatic limit of low ramp speeds (Góral et al., 2004; Ishkhanyan et al., 2004), i.e., when the parameter $1 /|\dot{B}|$ in Fig. 20 increases. We note that both the twolevel mean field approach and its associated Landau-Zener estimate of asymptotic populations do not distinguish between the sweep directions. Consequently, the molecule production in a downward sweep of the Feshbach resonance level is treated completely symmetrically to the heating of the gas in an upward sweep.

While Eqs. (132) and (133) refer to a genuinely two-level system, the two-level mean field approach accounts for the background scattering continuum, in principle, through the parameter $a_{\mathrm{bg}}$ of the mean field Hamiltonian of Eq. (138). Such a contact pseudo interaction treatment presupposes a separation between the typical time scales associated with the evolution of the Bose-Einstein condensate and the diatomic collisional duration (Köhler and Burnett, 2002; Proukakis et al., 1998). Accordingly, the two-level mean field approach can be derived formally in terms of the Markov limit of microscopic many-body theories of dilute gases (Góral et al., 2004) outlined in Subsection V.C. The assumption of a separation of time scales between two- and many-body evolutions is violated during a magnetic field sweep across a singularity of the scattering length. This implies that Eqs. (136) and (137) can describe the dynamics, at most, in the asymptotic regime where the dilute gas parameter, $\left[N|a(B)|^{3} / \mathcal{V}\right]^{1 / 2}$, is small compared to unity. Similarly to the limitations of the two-body Landau-Zener model, the intermediate evolution of a Bose-Einstein condensate is influenced by phenomena beyond the range of validity of the mean field approximation (Góral et al., 2004; Holland et al., 2001; Köhler et al., 2004). While most of the theoretical approaches agree in their predictions on the condensate loss in the fast sweep limit of Eq. (130), the saturation of molecule production is a matter of ongoing research (Naidon and Masnou-Seeuws, 2003, 2006).

\section{Molecule production in cold Bose and Fermi gases}

Cold Bose and two spin component Fermi gases are subject to a considerable momentum spread which generally tends to reduce the efficiency of molecule production via linear magnetic field sweeps across a zero energy resonance. Its significance is particularly obvious in the case of slow asymptotic sweeps, given the spectrum of dressed energies of an atom pair illustrated in Fig. 18 Only the $v=0$ mode adiabatically correlates with the Feshbach molecular level when the resonance energy is decreased, while all the excited states undergo cooling transitions. From this too simplistic viewpoint, for instance, two spin components of a dilute vapour of Fermi atoms distributed according to the Pauli exclusion principle would produce just a single molecule in the adiabatic limit of the ramp speed. Contrary to the case of BoseEinstein condensates, binary physics alone is therefore not even sufficient to qualitatively explain the observed substantial molecule production in such gases (Regal et al., 2003a; Strecker et al., 2003).

\section{Transitions from continuum to bound states}

A quantitative analysis of the problems associated with the theoretical description of molecule production in the presence of momentum spread may be based on the exact treatment of linear magnetic field sweeps of SubsectionIV.A Accordingly, the probability for transitions from an initial dressed continuum level of a pair of distinguishable atoms in a periodic box of volume $\mathcal{V}$ to the Feshbach molecular state at the final magnetic field strength is given by

$$
p_{\text {ass }}(\mathbf{k})=\frac{(2 \pi \hbar)^{3}}{\mathcal{V}}\left|\left\langle\phi_{\mathrm{b}}^{\mathrm{f}}\left|U_{2 \mathrm{~B}}\left(t_{\mathrm{f}}, t_{\mathrm{i}}\right)\right| \phi_{\hbar \mathbf{k}}^{\mathrm{i}}\right\rangle\right|^{2} .
$$

Here the arguments of the two-body time evolution operator, $t_{\mathrm{i}}$ and $t_{\mathrm{f}}$, refer to the initial and final times of the sweep, respectively, and $\hbar \mathbf{k}$ denotes the initial relative momentum of the atoms. Similarly to the derivation of Eq. (123), the asymptotic transition probability including the background scattering continuum can be determined analytically using the stationary phase approach in Eq. (141) in the limits $t_{\mathrm{i}} \rightarrow-\infty$ and $t_{\mathrm{f}} \rightarrow \infty$. This yields:

$$
p_{\text {ass }}(\mathbf{k})=\frac{(2 \pi \hbar)^{3}}{\mathcal{V}} \frac{2 \pi}{\hbar\left|\dot{E}_{\text {res }}\right|}\left|\left\langle\phi_{\text {res }}|W| \phi_{\hbar \mathbf{k}}^{(+)}\right\rangle\right|^{2} e^{-2 \operatorname{Im} \varphi\left(z_{\mathrm{i}}, t_{\mathrm{i}}\right)}
$$

Here $z_{\mathrm{i}}=\hbar^{2} k^{2} / m+i 0$ denotes the regularised energy argument of the initial phase. In accordance with Eqs. (110), (118) and (53), the exponent of Eq. (142) is determined by the formula

$$
\operatorname{Im} \varphi\left(z_{\mathrm{i}}, t_{\mathrm{i}}\right)=\frac{\pi \hbar^{2}}{\left|\dot{E}_{\text {res }}\right|} \int d \mathbf{k}^{\prime} \theta\left(k-k^{\prime}\right)\left|\left\langle\phi_{\text {res }}|W| \phi_{\hbar \mathbf{k}^{\prime}}^{(+)}\right\rangle\right|^{2} .
$$

Here the step function of the wave numbers, $\theta\left(k-k^{\prime}\right)$, indicates that transitions occur just in the intuitive, downward direction of an asymptotic sweep across $B_{0}$ (Demkov and Osherov, 1968). According to Eq. (143), the exponential damping of Eq. (142) increases in the limit $\left|\dot{E}_{\text {res }}\right| \rightarrow 0$. This confirms the intuitive picture suggested by Fig. 18 that adiabatic sweeps in a diatomic system with a continuum of modes eventually lead to negligible molecule production. While the momentum dependence of the exponent of Eq. (143) recovers experimental dissociation spectra (Mukaiyama et al., 2003), in the context of molecular association in cold gases, Eq. (142) gives rise to exact predictions just in the fast sweep limit.

\section{Fast sweep limit of molecule production}

The onset of molecule production in asymptotic magnetic field sweeps across zero energy resonances is sensitive to the 
statistics associated with identical atoms. SubsectionV.B. provides a strict approach to the determination of dimer populations on the basis of the two-particle correlation function of the gas. Similarly to the prediction of the loss of condensed atoms of Eq. (130), however, low depletions can be inferred intuitively from the fast sweep limit of Eq. (142), treating the gas as a reservoir of atom pairs. In this limit, $|\dot{B}| \rightarrow \infty$, the damping term of Eq. (143) describing cooling transitions into continuum levels below the initial energy $\hbar^{2} k^{2} / m$ vanishes. In the context of cold collisions in the Wigner threshold law regime the matrix element involving the inter-channel coupling in Eq. (142) may be evaluated at $\mathbf{k}=0$. Consequently, the transition probability of Eq. (142) becomes momentum independent and recovers the result of the Landau-Zener approach, $p_{\text {ass }}=2 \pi \delta_{\mathrm{LZ}}$.

For the purpose of studies involving $s$-wave collisions, Fermi gases are usually prepared as incoherent mixtures of two different Zeeman states with occupation numbers $N_{1}$ and $N_{2}$. Accordingly, $N=N_{1}+N_{2}$ is the total number of atoms. Each one of the $N_{1}$ atoms of the first component has $N_{2}$ atoms of the second component to interact with via $s$-wave collisions. Classical probability theory and the incoherent nature of the initial state therefore lead to the following estimate for the number of diatomic Feshbach molecules produced in the fast sweep limit (Chwedeńczuk et al., 2004):

$$
N_{\mathrm{d}}^{\mathrm{f}}=2 \pi N_{1} N_{2} \delta_{\text {LZ }}
$$

We note that the two-body Landau-Zener coefficient, given explicitly by the right hand side of Eq. (135), is inversely proportional to the volume $\mathcal{V}$ of the periodic box. The fraction of molecules, $N_{\mathrm{d}}^{\mathrm{f}} / N$, is therefore proportional to the density, $N / \mathcal{V}$, which allows for an extension of Eq. (144) to inhomogeneous gases via the local density approximation.

Given a balanced mixture of Zeeman states, i.e. $N_{1}=N_{2}=$ $N / 2$, Eqs. (144) and (131) show that the onset of molecule production in a Fermi gas is half as large as in a Bose-Einstein condensate of identical density and resonance parameters. In the limit of zero temperature such a dilute, initially weakly interacting vapour of atoms may be approximately described by a pair of filled Fermi seas. As fast magnetic field sweeps imply small atomic depletions, it may be argued intuitively that two-body cooling transitions leading into occupied modes below the Fermi energy are suppressed by the Pauli exclusion principle. This suggests a description of the two spin component Fermi sea in terms of a single level over a significant range of ramp speeds. The associated Landau-Zener approach (Chwedeńczuk et al., 2004) consistent with the linear limit of Eq. (144) is illustrated in Fig.21 in comparison to experiments on asymptotic magnetic field sweeps across the $543 \mathrm{G}$ zero energy resonance of ${ }^{6} \mathrm{Li}$ (Strecker et al., 2003). Accordingly, a suppression of cooling transitions gives an intuitive explanation for the onset of a substantial molecule production in two spin component Fermi gases.

In thermal Bose gases each one of the $N$ constituents can interact with all the other $N-1$ atoms to form a dimer Feshbach molecule. As opposed to the zero energy mode of condensed pairs, diatomic wave functions in the presence of momentum spread need to be explicitly symmetrised which enhances the

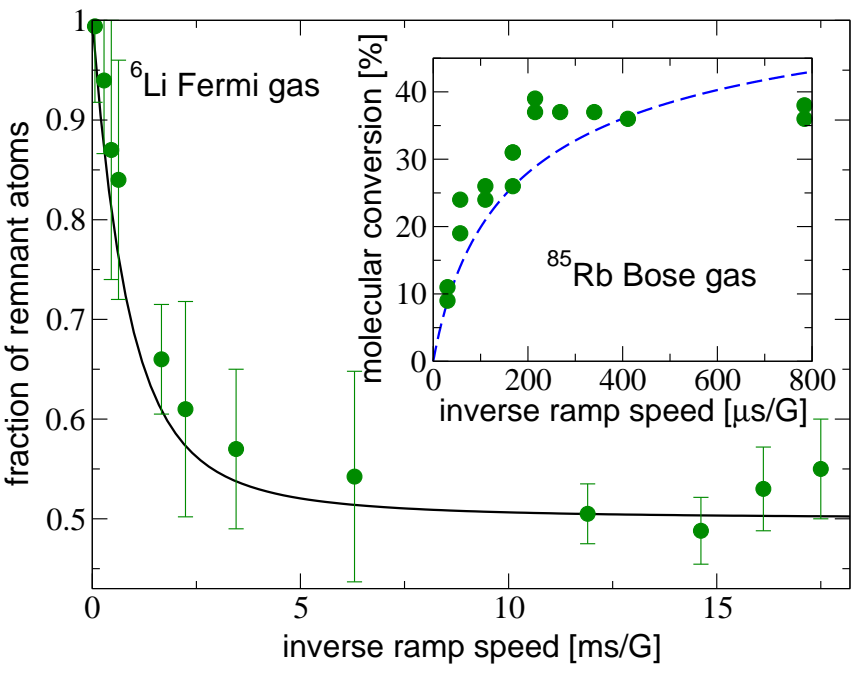

FIG. 21 (Colour in online edition) The fraction of remnant atoms, $1-2 N_{\mathrm{d}}^{\mathrm{f}} / N$, at the end of asymptotic magnetic field sweeps across the closed-channel dominated $543 \mathrm{G}$ zero energy resonance of ${ }^{6} \mathrm{Li}$ versus the inverse ramp speed (Strecker et al., 2003). The solid curve refers to a Landau-Zener estimate for the onset of molecule production in the fast sweep limit (Chwedeńczuk et al., 2004) using the local density approximation. The inset shows the molecular conversion, $2 N_{\mathrm{d}}^{\mathrm{f}} / N$, observed in a thermal Bose gas with a mean density of $1.3 \times 10^{11}$ atoms $/ \mathrm{cm}^{3}$ and a temperature of $40.6 \mathrm{nK}$ using magnetic field sweeps across the $155 \mathrm{G}$ zero energy resonance of ${ }^{85} \mathrm{Rb}$ (Hodby et al., 2005). For comparison, the dashed curve indicates the pairwise ensemble average over the transition probabilities, $2 p_{\text {ass }}(\mathbf{k})$, with respect to the Maxwell distribution of relative velocities. This semi-classical estimate for the onset of molecule production in Bose gases is based on Eq. (142) and therefore consistent with the limit of Eq. (145). Both the solid and dashed curves are associated with the parameters $a_{\mathrm{bg}}$ and $\Delta B$ of Tables $[\mathrm{IV}$ and $\mathrm{V}$ respectively.

association probability of Eq. (141) in the thermal average by a factor of two (Stoof et al., 1989). As the number of interacting pairs is $N(N-1) / 2 \approx N^{2} / 2$, the fast sweep limit of the number of Feshbach molecules produced in a thermal Bose gas is given by the following formula:

$$
N_{\mathrm{d}}^{\mathrm{f}}=2 \pi N^{2} \delta_{\mathrm{LZ}} .
$$

This estimate is illustrated in the inset of Fig. 21 in comparison to experiments associated with thermal clouds of ${ }^{85} \mathrm{Rb}$ (Hodby et al., 2005). We note that a similar enhancement of dimer formation in thermal Bose gases as compared to condensates was observed in the context of inelastic three-body recombination and reflects correlation properties (Burt et al., 1997; Kagan et al., 1985).

According to Eqs. (144), (131) and (145), the onsets of molecule production increase by factors of two between balanced two spin component Fermi gases, Bose-Einstein condensates, and thermal Bose gases of identical densities and resonance parameters. The validity of these statistical estimates based on two-body physics depends on the significance of multiple collisions of each atom during a magnetic field sweep. This implies that the range of ramp speeds described by the fast sweep limits depends on the density 
of the gas in addition to the specific nature of the Feshbach molecules associated with entrance- and closed-channel dominated zero energy resonances. Predictions accessing wider ranges of ramp speeds require genuinely many-body approaches (Javanainen et al., 2004; Pazy et al., 2005, 2004; Williams et al., 2004a.b).

\section{Saturation of molecule production}

Magnetic field sweeps sufficiently slow to convert up to $88 \%$ of the atoms into Feshbach molecules were employed to produce Bose-Einstein condensates of dimers from balanced two spin component mixtures of ${ }^{40} \mathrm{~K}$ Fermi atoms (Greiner et al., 2003). Figure4 shows a typical density profile of such a final molecular cloud (right image) in comparison to a thermal distribution (left image). Motivated by the theory of ideal Bose gases, the conditions for condensation in these experiments are likely to depend on the phase space density, $n_{\mathrm{a}}^{\mathrm{i}} \lambda_{\text {th }}^{3}$, associated with the initial dilute atomic gas of density $n_{\mathrm{a}}^{\mathrm{i}}$ and temperature $T$. Here $\lambda_{\mathrm{th}}=\left[2 \pi \hbar^{2} /\left(m k_{\mathrm{B}} T\right)\right]^{1 / 2}$ is the thermal de Broglie wavelength. Given the experimental densities on the order of $10^{13}$ atoms per $\mathrm{cm}^{3}$ and the $202 \mathrm{G}$ zero energy resonance parameters, magnetic field sweeps with low ramp speeds of typically $160 \mathrm{G} / \mathrm{s}$ (Greiner et al., 2003) may be considered to be adiabatic. Such processes smoothly alter diatomic wave functions but are not necessarily expected to change the occupation of states in phase space. It is therefore plausible that not only the condition for molecular condensation but also the saturated production efficiency, $2 N_{\mathrm{d}}^{\mathrm{f}} / N$, is determined just by the phase space density rather than the number of atoms or their temperature individually.

Subsequent systematic studies of adiabatic magnetic field sweeps in both two spin component Fermi gases of ${ }^{40} \mathrm{~K}$ and thermal Bose gases of ${ }^{85} \mathrm{Rb}$ have supported this view (Hodby et al. , 2005). The measured molecule production efficiencies of Fig. 22 were analysed in terms of a stochastic model relying upon the assumption that the probability for two atoms to form a dimer depends solely on their proximity in phase space. Based on the experimental data, the associated proximity conditions turned out to be virtually identical for both atomic species, ${ }^{40} \mathrm{~K}$ and ${ }^{85} \mathrm{Rb}$. These observations can be understood from first principles using coupled Boltzmann equations for the Wigner functions associated with density matrices of separated atoms and pairs in the resonance state configuration (Williams et al., 2006). Figure 22 illustrates the accuracy of this approach including quantum statistical effects (Bloch, 1928; Uehling and Uhlenbeck, 1933) as well as its classical gas limit.

Similar experiments using adiabatic magnetic field sweeps across the $834 \mathrm{G}$ zero energy resonance in cold two spin component Fermi gases of ${ }^{6} \mathrm{Li}$ yielded up to $80 \%$ Feshbach molecular conversion (Cubizolles et al., 2003). Such observations were analysed in terms of theoretical approaches based on the assumption of thermal equilibrium throughout the sweep (Chin and Grimm, 2004; Kokkelmans et al., 2004). In the magnetic field range of positive scattering lengths about $B_{0}$ the Feshbach molecular level gives rise to a local minimum

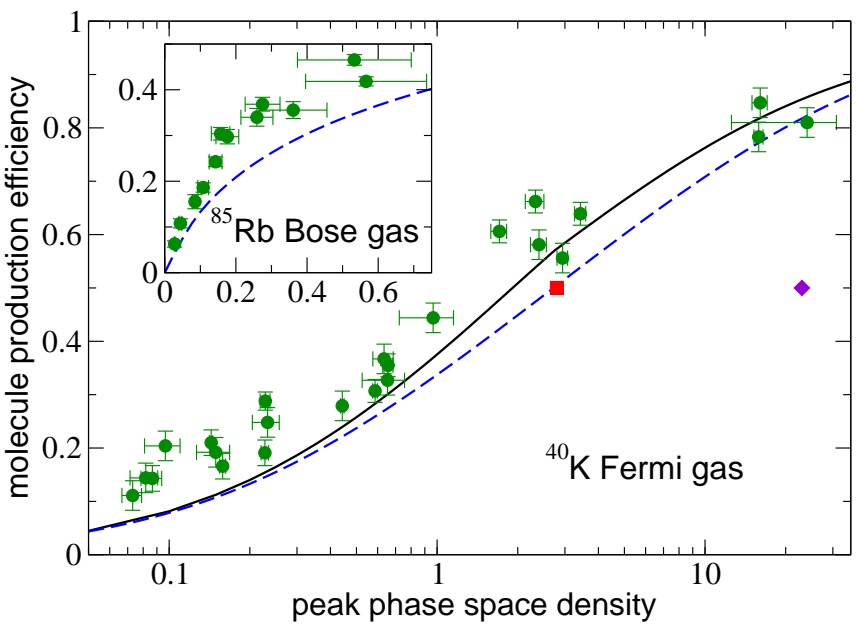

FIG. 22 (Colour in online edition) The molecule production efficiency, $2 N_{\mathrm{d}}^{\mathrm{f}} / N$, versus the peak phase space density of cold Bose as well as two spin component Fermi gases. The circles refer to dimer populations measured at the end of adiabatic magnetic field sweeps across the zero energy resonances of ${ }^{85} \mathrm{Rb}$ at $155 \mathrm{G}$ (inset) and of ${ }^{40} \mathrm{~K}$ at $202 \mathrm{G}$ (Hodby et al., 2005). The curves indicate predictions (Williams et al., 2006) based on coupled Boltzmann equations including quantum statistical effects (solid curve) as well as their associated classical gas limit (dashed curves). For comparison, the square and the diamond refer to the molecular production efficiencies observed for the lowest ramp speeds in earlier sweep experiments using zero energy resonances of ${ }^{40} \mathrm{~K}$ at $224 \mathrm{G}$ (Regal et al., 2003a) and of ${ }^{6} \mathrm{Li}$ at $543 \mathrm{G}$ (Strecker et al., 2003), respectively.

of the many-particle action (Szymańska et al., 2005) which is associated with a meta-stable state. As this state is energetically favourable to a gas of separated atoms, it may be argued intuitively that production of dimer molecules should occur eventually also at a stationary magnetic field strength when the system equilibrates. This principle has been employed to convert balanced incoherent two spin component mixtures of ${ }^{6} \mathrm{Li}$ atoms into dilute vapours of Feshbach molecules (Jochim et al., 2003a). While energy conservation inevitably leads to components of comparatively hot atoms and dimers, the stability of universal Feshbach molecules discussed in Subsection $\amalg$ III.E allows such gases to be cooled by evaporation. The associated increase in phase space density provides an alternative route to the Bose-Einstein condensation of ${ }^{6} \mathrm{Li}_{2}$ (Jochim et al., 2003b; Zwierlein et al., 2003) besides the approach of adiabatic magnetic field sweeps.

\section{Dissociation of Feshbach molecules}

Asymptotic upward sweeps of the resonance energy lead to the dissociation of Feshbach molecules which often serves as a precursor to their detection. To this end, a molecular component is usually spatially separated from the environment of a remnant atomic gas using, for instance, the SternGerlach technique illustrated in Section III.G. After this separation the dissociation allows the fragments to be detected conventionally using probe lasers tuned to resonance with an 
atomic spectral line. The energy provided by the time varying homogeneous magnetic field during the sweep is transferred to the relative motion of the constituents of a Feshbach molecule. Such correlated atom pairs with a relative velocity depending on the ramp speed were detected in several experiments (Dürr et al., 2004b; Mukaiyama et al., 2003; Volz et al., 2005). Their spectrum of kinetic energies of the relative motion is given by the following expression:

$$
n_{\mathrm{diss}}\left(\hbar^{2} k^{2} / m\right)=\frac{m \hbar k}{2} \int d \Omega\left|\left\langle\phi_{\hbar \mathbf{k}}^{\mathrm{f}}\left|U_{2 \mathrm{~B}}\left(t_{\mathrm{f}}, t_{\mathrm{i}}\right)\right| \phi_{\mathrm{b}}^{\mathrm{i}}\right\rangle\right|^{2} .
$$

Here $d \Omega$ denotes the angular component of $d \mathbf{k}$ describing the direction of the momentum $\hbar \mathbf{k}$, while $\left|\phi_{\mathrm{b}}^{\mathrm{i}}\right\rangle$ and $\left|\phi_{\hbar \mathbf{k}}^{\mathrm{f}}\right\rangle$ are bound and dressed continuum states associated with the initial and final magnetic field strengths, respectively.

Similarly to the derivation of Eqs. (142) and (143), the energy spectrum of Eq. (146) can be determined analytically in the asymptotic limits $t_{\mathrm{i}} \rightarrow-\infty$ and $t_{\mathrm{f}} \rightarrow \infty$. As dissociation and association are related to each other just by time reversal, their transition probability densities are identical. Typical energies $\hbar^{2} k^{2} / m$ of atomic fragments are on the order of $\mu \mathrm{K}$ in units of the Boltzmann constant which is usually inside the Wigner threshold law regime, i.e. $k\left|a_{\mathrm{bg}}\right| \ll 1$. This implies that the matrix elements involving the inter-channel coupling in Eqs. (142) and (143) can be evaluated at $\mathbf{k}=0$ and $\mathbf{k}^{\prime}=0$, respectively. Consequently, the asymptotic dissociation spectrum of Eq. (146) is well approximated by the following formula (Góral et al., 2004; Mukaiyama et al., 2003):

$$
n_{\mathrm{diss}}(E)=-\frac{\partial}{\partial E} \exp \left(-\frac{4}{3} \sqrt{\frac{m E}{\hbar^{2}}} \frac{\left|a_{\mathrm{bg}} \Delta B\right| E}{\hbar|\dot{B}|}\right) .
$$

We note that the integral of Eq. (147) over all kinetic energies $E$ of the relative motion gives unity. This implies that Feshbach molecules are dissociated with certainty in an asymptotic upward sweep of the resonance energy, provided that transitions to the highest excited entrance channel vibrational level are negligible.

According to Eq. (147), the width of the dissociation spectrum increases with increasing ramp speeds. The associated single particle kinetic energies are usually inferred from the velocities of the fragments which constitute a radially expanding cloud of atoms. Their average, $E_{\text {diss }}$, amounts to one half of the mean energy of the relative motion of all correlated pairs given in terms of Eq. (146) by the expression

$$
E_{\mathrm{diss}}=\frac{1}{2} \int_{0}^{\infty} d E E n_{\mathrm{diss}}(E) .
$$

Accordingly, the Wigner threshold law approximation of Eq. (147) determines $E_{\text {diss }}$ to be

$$
E_{\mathrm{diss}}=\frac{1}{3}\left(\frac{3}{4} \sqrt{\frac{\hbar^{2}}{m a_{\mathrm{bg}}^{2}}} \frac{\hbar|\dot{B}|}{|\Delta B|}\right)^{2 / 3} \Gamma(2 / 3) .
$$

Figure 23 illustrates the accuracy of the predictions of Eq. (149) on average dissociation energies detected at the end

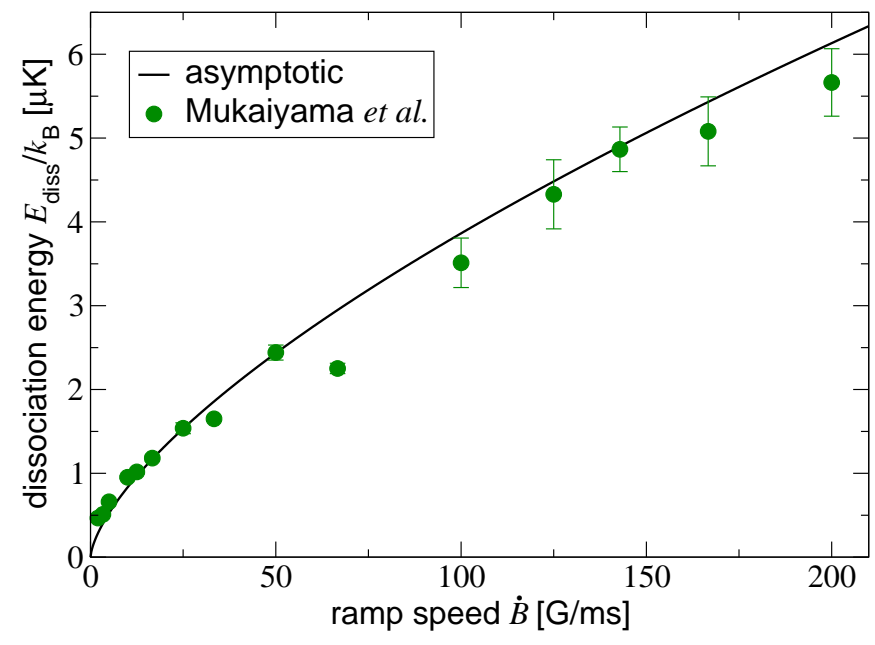

FIG. 23 (Colour in online edition) The mean energies of the atomic fragments of ${ }^{23} \mathrm{Na}_{2}$ Feshbach molecules dissociated by a linear upward sweep of the $907 \mathrm{G}$ resonance level versus the ramp speed. Circles indicate experimental data (Mukaiyama et al., 2003), while the solid curve refers to the predictions of Eq. (149) using the parameters $a_{\mathrm{bg}}$ and $\Delta B$ of Tables [V] and $\mathrm{V}$ respectively.

of asymptotic magnetic field sweeps across the $907 \mathrm{G}$ zero energy resonance of sodium (Mukaiyama et al., 2003). While the solid curve refers to independently determined parameters $a_{\mathrm{bg}}$ and $\Delta B$ (Mies et al., 2000), the good agreement with the measurements has motivated experiments using molecular dissociation to characterise resonances (Brouard and Plata, 2005; Dürr et al., 2004b).

We note that the asymptotic energy spectrum of Eq. (147) depends just on the product $a_{\mathrm{bg}} \Delta B$ which, according to Eq. (39), determines near resonant scattering lengths in the universal regime of magnetic field strengths. A similar statement applies to all Landau-Zener coefficients as well as the long time asymptotic occupations predicted by the two-level mean field approach (Góral et al., 2004). In the limits $t_{\mathrm{i}} \rightarrow$ $-\infty$ and $t_{\mathrm{f}} \rightarrow \infty$ the number of correlated atom pairs produced by linear magnetic field sweeps is, therefore, insensitive to the parameters $\mu_{\text {res }}$ and $B_{0}-B_{\text {res }}$ which directly refer to properties of the resonance level. This suggests that such populations can be inferred from any one of the single channel approaches outlined in Subsections III.F and III.G which all give the correct magnetic field dependence of the scattering length (Góral et al., 2004; Köhler et al., 2004). All derivations of the exact diatomic dynamics associated with linear magnetic field sweeps of Subsection IV.A may be performed, for instance, using a single channel Hamiltonian with a time dependent separable potential. Despite the fact that such a two-body interaction is not suited to describe closed-channel dominated Feshbach molecular states, it exactly recovers the asymptotic dissociation spectrum of Eq. (147).

In accordance with Eq. (122), the range of validity of the limits $t_{\mathrm{i}} \rightarrow-\infty$ and $t_{\mathrm{f}} \rightarrow \infty$ in applications to linear sweeps is determined by the accuracy of the stationary phase approach. Given a fixed ramp speed, this approximation is violated when the sweep starts or terminates too close to $B_{0}$ for the variation 
of the phases in Eq. (113) to produce sufficiently many oscillations of the complex exponential functions. We note that according to Eq. (119) the phase gradient with respect to the energy parameter is proportional to $1 / \dot{B}$. This implies that slow sweeps tend to require smaller ranges of magnetic field strengths about $B_{0}$ for the asymptotic limits to be applicable. Conversely, the faster a sweep the more it resolves details of both the intermediate dynamics and the initial and final states.

In the idealised limit of a jump of the magnetic field strength across $B_{0}$ the Feshbach molecular dissociation spectrum of Eq. (146) just probes the overlap of the dressed initial bound and final continuum states. Such a scenario has been realised using a narrow closed-channel dominated zero energy resonance of ${ }^{87} \mathrm{Rb}$ (Dürr et al., 2004b). In these experiments the initial magnetic field strength was chosen in such a way that the Feshbach molecule was virtually identical to the resonance state, $\left|\phi_{\mathrm{res}}, \mathrm{cl}\right\rangle$. According to Eq. (33), this implies that the dissociation spectrum is determined simply by the modulus squared of the amplitude of Eq. (34), i.e. $n_{\text {diss }}\left(p^{2} / m\right) 2 /(m p)=4 \pi\left|A\left(B_{\mathrm{f}}, p^{2} / m\right)\right|^{2}$. Its resonance denominator gives rise to a sharp maximum at $E_{\mathrm{res}}\left(B_{\mathrm{f}}\right)$ (Dürr et al., 2004b; Haque and Stoof, 2005) slightly shifted by the real part of Eq. (54), while the associated imaginary part yields the spectral width. Such dissociation jumps across $B_{0}$ using a resonance state of $d$-wave symmetry can populate several outgoing partial waves of the atomic fragments. This approach was employed, for instance, for the spectroscopy of a $d$-wave shape resonance (Dürr et al., 2005; Volz et al., 2005).

\section{ATOM-MOLECULE COHERENCE}

Besides the production of Feshbach molecules via asymptotic linear sweeps across $B_{0}$, several approaches to date rely upon ramp sequences (Mark et al., 2005; Yurovsky and Ben-Reuven, 2005) or resonant oscillating magnetic fields (Thompson et al., 2005a). Such experimental techniques are designed to improve the efficiency of dimer formation in Bose gases mainly by avoiding the region of large scattering lengths and accordingly strong inter-atomic interactions. The associated nonlinear magnetic field variations lead to dependences of the molecular population on properties of zero energy resonances beyond those included in the LandauZener parameter.

\section{A. Ramsey interferometry with atoms and molecules}

Pulses starting and ending on the positive scattering length side of a zero energy resonance (Claussen et al., 2002) are of particular interest in this context. Their repeated application to a ${ }^{85} \mathrm{Rb}$ Bose-Einstein condensate gives rise to the coherent oscillations between the final components of separated atoms and Feshbach molecules of Fig.2 (Claussen et al., 2003; Donley et al., 2002).

\section{Magnetic field pulse sequence}

A typical experimental pulse sequence (Donley et al., 2002) is illustrated in Fig. 24 All the associated magnetic field strengths, $B$, are on the high field side of the $155 \mathrm{G}$ zero energy resonance of ${ }^{85} \mathrm{Rb}$ where the scattering length is positive. Consequently, the inter-atomic potential supports a loosely bound dimer state whose energy as a function of $B$ is shown in Fig. 12 The conversion of pairs of BoseEinstein condensed atoms to Feshbach molecules in these experiments crucially relies upon intermediate order of magnitude variations of the scattering length, $a(B)$, and, accordingly, of the dimer bond length, $\langle r\rangle \approx a(B) / 2$. The pulse sequence of Fig. 24 starts at the evaporation field strength of about $162 \mathrm{G}$ at which a dilute ${ }^{85} \mathrm{Rb}$ Bose-Einstein condensate is usually produced (Cornish et al., 2000) with a scattering length of about $200 a_{\text {Bohr }}$. Given the experimental mean inter-atomic distance of about $12000 a_{\mathrm{Bohr}}$, the gas is therefore initially weakly interacting. Each pulse tunes the scattering length to $a\left(B_{\min }\right) \approx 9000 a_{\text {Bohr }}$ on time scales too short for the Bose-Einstein condensate to adjust its density. Consequently, at the magnetic field strength $B_{\min }$ closest to the zero energy resonance the gas is driven into the regime of strong inter-atomic interactions. During the course of the experiments (Claussen et al., 2003; Donley et al., 2002), the stationary field, $B_{\text {evolve }}$, associated with the evolution period separating the pulses as well as the duration $t_{\text {evolve }}$ were varied. The variation of $B_{\text {evolve }}$ between $156 \mathrm{G}$ and $162 \mathrm{G}$ corresponds to the range of Feshbach molecular binding energies displayed in the inset of Fig.12 with associated scattering lengths between about $4000 a_{\text {Bohr }}$ and $200 a_{\text {Bohr }}$.

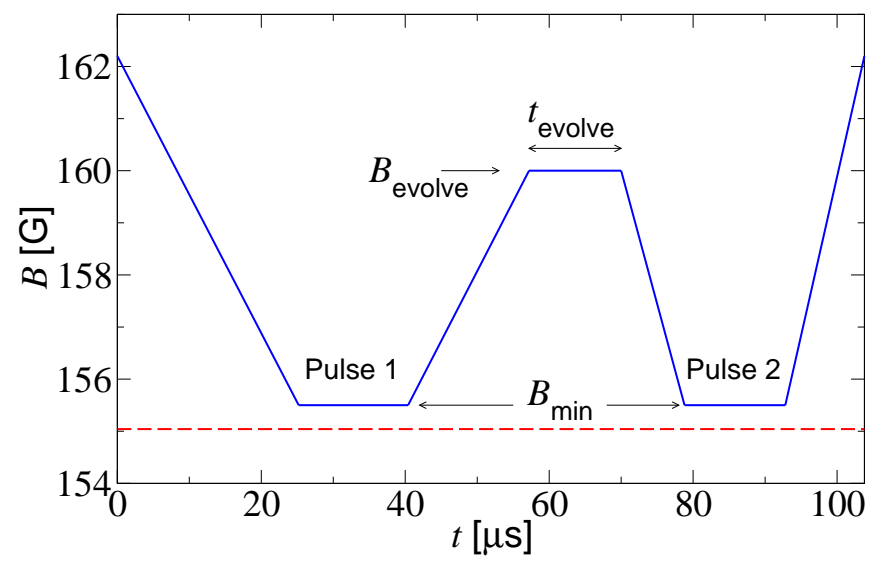

FIG. 24 (Colour in online edition) Scheme of a typical magnetic field pulse sequence (solid lines) employed in experiments producing coherent oscillations between final atomic and molecular components in a ${ }^{85} \mathrm{Rb}$ Bose-Einstein condensate (Donley et al., 2002). A pair of pulses with a minimum field strength, $B_{\min }=155.5 \mathrm{G}$, is separated by an evolution period of variable duration, $t_{\text {evolve }}$, and field strength, $B_{\text {evolve }}$. The zero energy resonance position, $B_{0} \approx 155 \mathrm{G}$, is indicated by the dashed line.

All measurements probing the densities of atoms were performed after each pulse sequence had terminated and therefore reflect the state of the weakly interacting gas at the final 
magnetic field strength of about $162 \mathrm{G}$ in Fig. 24, According to these observations, the fast magnetic field variation gives rise to the three components of the atomic cloud illustrated in Fig. 22 The occupation numbers associated with the remnant Bose-Einstein condensate, burst component and undetected atoms all oscillate with respect to each other as a function of the evolution time, $t_{\text {evolve }}$. It turned out that their common angular frequency, $\omega_{\mathrm{e}}$, is accurately determined by the Feshbach molecular energy in the evolution period, $E_{\mathrm{b}}^{\text {evolve }}$, via the relation $\omega_{\mathrm{e}}=\left|E_{\mathrm{b}}^{\mathrm{evolve}}\right| / \hbar$. Donley et al. concluded that the undetected atoms were transferred into Feshbach molecules whose fast phase evolution as compared to the atomic components leads to interference fringes in the final occupations.

In accordance with such an intuitive explanation, the first pulse provides the overlap between the dimer size and the average distance between the condensed atoms which is crucial to the molecular association. As this fast magnetic field variation is not resonant with the binding energy, it inevitably leads to the additional production of excited atom pairs which constitute a burst of atoms. During the evolution period all components of the weakly interacting gas evolve independently and coherently and therefore accumulate a phase difference of $\Delta \varphi=\omega_{\mathrm{e}} t_{\mathrm{evolve}}$. Finally, the second pulse gets the Feshbach molecules and separated atoms to overlap once again and thereby probes $\Delta \varphi$ via an interference in their occupations. This intuitive scenario is analogous to the principle of a Ramsey interferometer using pairs of separated atoms and Feshbach molecules (Donley et al., 2002; Zoller, 2002) whose scheme is illustrated in Fig. 25.

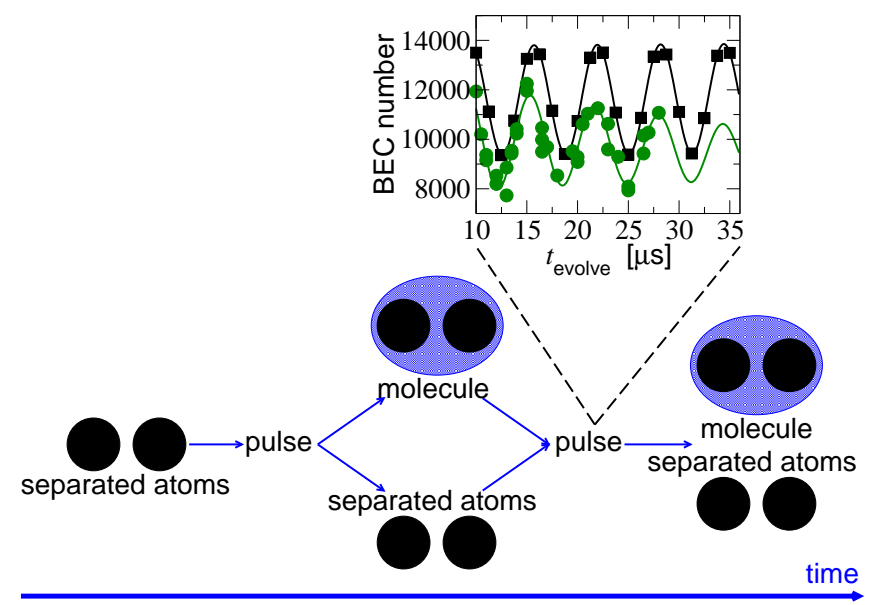

FIG. 25 (Colour in online edition) Schematic illustration of the Ramsey interferometry with ${ }^{85} \mathrm{Rb}$ atoms and Feshbach molecules associated with the magnetic field pulse sequence of Fig. 24 (Donley et al., 2002; Zoller, 2002). The first pulse drives the initial Bose-Einstein condensate into a coherent superposition of bound and unbound atom pairs. Between the pulses the individual orthogonal components evolve independently. The second pulse gets the atomic and molecular states to overlap and thereby probes their phase difference. The inset shows a typical interference fringe pattern at the end of the pulse sequence as a function of the evolution time, $t_{\text {evolve }}$. Circles refer to measurements of the remnant Bose-Einstein condensate (Claussen et al., 2003), while squares indicate theoretical predictions (Góral et al., 2005).

\section{Dynamics of a single atom pair}

In analogy to the analysis of Subsection IV.C, the observations of Fig. 2 can be qualitatively understood in terms of the dynamics of just a single pair of atoms (Borca et al., 2003; Góral et al., 2005) in a periodic box of volume $\mathcal{V}$. To this end, the two-body time evolution operator of Eq. (98) associated with the magnetic field variation of Fig. 24 may be split into its contributions of the first and second pulse and of the evolution period. This yields:

$$
U_{2 \mathrm{~B}}\left(t_{\mathrm{f}}, t_{\mathrm{i}}\right)=U_{2}\left(t_{\mathrm{f}}, t_{2}\right) U_{\text {evolve }}\left(t_{\text {evolve }}\right) U_{1}\left(t_{1}, t_{\mathrm{i}}\right) .
$$

Here the first pulse starts at the initial time $t_{\mathrm{i}}$ and terminates at $t_{1}$, while $t_{2}=t_{1}+t_{\text {evolve }}$ indicates the beginning of the second pulse which ends at the final time of the sequence, $t_{\mathrm{f}}$. Accordingly, $U_{1}\left(t_{1}, t_{\mathrm{i}}\right)$ and $U_{2}\left(t_{\mathrm{f}}, t_{2}\right)$ describe the two-body dynamics during the first and second pulse, respectively. In between the pulses the magnetic field strength is stationary, in accordance with Fig. 24. This implies that the associated time evolution operator, $U_{\text {evolve }}\left(t_{\text {evolve }}\right)$, depends just on the duration $t_{\text {evolve }}$. Its spectral decomposition in terms of dressed states of the relative motion of an atom pair exposed to the magnetic field of strength $B_{\text {evolve }}$ is given by the following formula:

$$
U_{\text {evolve }}\left(t_{\text {evolve }}\right)=\sum_{v=-1}^{\infty}\left|\phi_{v}^{\text {evolve }}\right\rangle e^{-i E_{v}^{\text {evolve }} t_{\text {evolve }} / \hbar}\left\langle\phi_{v}^{\text {evolve }}\right| .
$$

Here the index $v$ labels the vibrational quantum numbers of the box states in such a way that $\left|\phi_{-1}^{\text {evolve }}\right\rangle$ correlates adiabatically, in the limit of infinite volume $\mathcal{V}$, with the Feshbach molecular dressed state, $\left|\phi_{\mathrm{b}}^{\text {evolve }}\right\rangle$. Accordingly, the associated vibrational energy levels are denoted by $E_{v}^{\text {evolve }}$ which implies the asymptotic behaviour $E_{-1}^{\text {evolve }} \sim E_{\mathrm{b}}^{\text {evolve }}$ with increasing $\mathcal{V}$. The more tightly bound states associated with vibrational quantum numbers $v<-1$ contribute negligibly to the fringe pattern of Fig. 25 and are therefore omitted in Eq. (151).

Similarly to the treatment of Subsection IV.C the interference fringes in the population of the remnant condensate component shown in the inset of Fig. 25 can be described in terms of the following transition probability:

$$
p_{0,0}=\left|\left\langle\phi_{0}^{\mathrm{f}}\left|U_{2 \mathrm{~B}}\left(t_{\mathrm{f}}, t_{\mathrm{i}}\right)\right| \phi_{0}^{\mathrm{i}}\right\rangle\right|^{2} .
$$

To this end, it is instructive to split the associated amplitude, in accordance with Eq. (151), into its contributions of the vibrational states with quantum numbers $v \geq 0$ and of the Feshbach molecule. This yields:

$$
\left\langle\phi_{0}^{\mathrm{f}}\left|U_{2 \mathrm{~B}}\left(t_{\mathrm{f}}, t_{\mathrm{i}}\right)\right| \phi_{0}^{\mathrm{i}}\right\rangle=D_{2 \mathrm{~B}}+A_{2 \mathrm{~B}} .
$$

Due to the quasi continuum of levels, the first term, $D_{2 \mathrm{~B}}$, on the right hand side of Eq. (153) tends to decay via phase diffusion as a function of $t_{\text {evolve }}$. Its explicit expression reads:

$$
\begin{aligned}
D_{2 \mathrm{~B}}= & \sum_{v=0}^{\infty}\left\langle\phi_{0}^{\mathrm{f}}\left|U_{2}\left(t_{\mathrm{f}}, t_{2}\right)\right| \phi_{v}^{\text {evolve }}\right\rangle e^{-i E_{v}^{\text {evolve }} t_{\text {evolve }} / \hbar} \\
& \times\left\langle\phi_{v}^{\text {evolve }}\left|U_{1}\left(t_{1}, t_{\mathrm{i}}\right)\right| \phi_{0}^{\mathrm{i}}\right\rangle .
\end{aligned}
$$


The magnitude of the second term, $A_{2 \mathrm{~B}}$, on the right hand side of Eq. 153 depends on the product of the amplitudes for Feshbach molecular association during the first pulse and dissociation into the zero mode due to the second pulse. Its evolution as a function of $t_{\text {evolve }}$ stems just from the phase shift associated with the energy $E_{-1}^{\text {evolve }}$, in accordance with the following formula:

$$
\begin{aligned}
A_{2 \mathrm{~B}}= & \left\langle\phi_{0}^{\mathrm{f}}\left|U_{2}\left(t_{\mathrm{f}}, t_{2}\right)\right| \phi_{-1}^{\text {evolve }}\right\rangle e^{-i E_{-1}^{\text {evolve }} t_{\text {evolve }} / \hbar} \\
& \times\left\langle\phi_{-1}^{\text {evolve }}\left|U_{1}\left(t_{1}, t_{\mathrm{i}}\right)\right| \phi_{0}^{\mathrm{i}}\right\rangle .
\end{aligned}
$$

The modulus squared of the sum of interfering amplitudes, $D_{2 \mathrm{~B}}$ and $A_{2 \mathrm{~B}}$, therefore leads to the anticipated fringe pattern in its usual form,

$$
p_{0,0}=\left|D_{2 \mathrm{~B}}\right|^{2}+\left|A_{2 \mathrm{~B}}\right|^{2}+2\left|D_{2 \mathrm{~B}}\right|\left|A_{2 \mathrm{~B}}\right| \sin \left(\omega_{\mathrm{e}} t_{\text {evolve }}+\varphi\right)
$$

in agreement with the illustration of Fig. 25. Here the angular frequency, $\omega_{\mathrm{e}}=\left|E_{-1}^{\mathrm{evolve}}\right| / \hbar$, is determined by the Feshbach molecular binding energy. The absolute phase, $\varphi$, is associated with both amplitudes $A_{2 \mathrm{~B}}$ and $D_{2 \mathrm{~B}}$ and depends sensitively on the exact shape of each pulse. A similar statement applies to the efficiency of dimer production and dissociation which are both crucial to the fringe visibility, according to Eqs. (155) and (156).

The populations of Feshbach molecules as well as the burst spectrum composed of the excited dressed levels at the final magnetic field strength may be inferred from the associated transition probabilities, similarly to Eq. (156). Their pairwise averages in a volume $\mathcal{V}$ in addition to a subsequent local density approximation give a good account of the magnitudes of all components of the gas shown in Fig. 2 (Góral et al., 2005). The range of validity of such a two-body estimate is set by the requirement that $B_{\text {evolve }}$ is sufficiently far from $B_{0}$ that the mean distance between the atoms is much larger than the scattering length during the evolution period. As the $155 \mathrm{G}$ zero energy resonance of ${ }^{85} \mathrm{Rb}$ is entrance-channel dominated, the two-body dynamics is well described in terms of both the effective two- and single-channel approaches of Subsection II.G Góral et al., 2005; Köhler et al., 2003a). We note, however, that the contact pseudo interaction is insufficient. This is immediately apparent, for instance, from the inaccuracy of the universal estimate of the binding energies in the inset of Fig. 12 whose experimental data were determined using measured fringe frequencies (Claussen et al., 2003). The interference experiments are therefore sensitive to the van der Waals tail of the inter-atomic potential which is not accounted for by the Landau-Zener parameter associated with asymptotic linear magnetic field sweeps.

\section{B. Number of dimers produced in Bose and Fermi gases}

Another significant difference in the descriptions of the linear sweep and magnetic field pulse techniques is associated with the treatment of the Feshbach molecular state. The results of Subsection IV.A suggest that the number of dimers produced via asymptotic magnetic field sweeps may be inferred from two-level approaches effectively identifying dressed Feshbach molecules with bare resonance states. For the Ramsey interference experiments (Claussen et al., 2003; Donley et al., 2002) operating just in the vicinity of the zero energy resonance a maximum final molecular conversion of about $16 \%$ was reported under the conditions referred to in Fig. 2] The closed channel admixture to the dressed bound state, however, is approximately $25 \%$ at the final magnetic field strength of $162 \mathrm{G}$ (Köhler et al., 2004) and even smaller throughout the pulse sequence (Braaten et al., 2003; Köhler et al., 2003b). In order to predict the observed number of dimers, it is therefore necessary to include the long range nature of the Feshbach molecules produced in the description of their detection.

An associated generic approach suitable for both magnetic field sweeps and pulse sequences may be based on the following observable for the population of any diatomic state, $\left|\phi_{\mathrm{d}}\right\rangle$, in a gas consisting of $N$ atoms (Köhler et al., 2003a):

$$
\mathrm{N}_{\mathrm{d}}=\frac{1}{2} \sum_{\substack{i, j=1 \\ i \neq j}}^{N}\left|\phi_{i j}^{\mathrm{d}}\right\rangle\left\langle\phi_{i j}^{\mathrm{d}}\right| .
$$

Here the indices $i$ and $j$ label the atoms, $\left|\phi_{i j}^{\mathrm{d}}\right\rangle$ refers to the two-body state, $\left|\phi_{\mathrm{d}}\right\rangle$, associated with the atoms $i$ and $j$, and the factor of $1 / 2$ on the right hand side of Eq. 157 prevents double counting of pairs. Given any state of the gas generally described by a density matrix, $\rho_{N \mathrm{~B}}$, the number of atomic pairs in the state $\left|\phi_{\mathrm{d}}\right\rangle$ is consequently determined by the following expectation value:

$$
N_{\mathrm{d}}=\left\langle\mathrm{N}_{\mathrm{d}}\right\rangle=\operatorname{Tr}\left(\mathrm{N}_{\mathrm{d}} \rho_{N \mathrm{~B}}\right) .
$$

Here the symbol "Tr" refers to the trace over the degrees of freedom of all atoms constituting the gas.

Many-body systems consisting of identical atoms are usually described using the approach of second quantisation (Fetter and Walecka, 1971). Accordingly, any $N$ particle state is constructed by repeated application of creation operators to the vacuum, $|\Omega\rangle$, which refers to the absence of atoms. The associated field operators, $\psi_{\alpha}(\mathbf{x})$ and $\psi_{\beta}^{\dagger}(\mathbf{y})$, annihilating and creating single atoms at the positions $\mathbf{x}$ and $\mathbf{y}$, respectively, fulfil the following (anti-)commutation relations:

$$
\psi_{\alpha}(\mathbf{x}) \psi_{\beta}^{\dagger}(\mathbf{y}) \mp \psi_{\beta}^{\dagger}(\mathbf{y}) \psi_{\alpha}(\mathbf{x})=\delta_{\alpha \beta} \delta(\mathbf{x}-\mathbf{y}) .
$$

Here the minus sign is associated with bosons while the plus sign refers to fermions, and the Greek labels $\alpha$ and $\beta$ indicate their Zeeman states. Given the diatomic wave function of the relative motion, $\phi_{\alpha \beta}^{\mathrm{d}}(\mathbf{r})=\left\langle\mathbf{r} ; \alpha, \beta \mid \phi_{\mathrm{d}}\right\rangle$, the second quantised representation of the number operator of Eq. 157 reads:

$$
\begin{gathered}
\mathrm{N}_{\mathrm{d}}=\frac{1}{2} \sum_{\alpha, \beta, \alpha^{\prime}, \beta^{\prime}} \int d \mathbf{R} \int d \mathbf{r} \int d \mathbf{r}^{\prime} \phi_{\alpha \beta}^{\mathrm{d}}(\mathbf{r})\left[\phi_{\alpha^{\prime} \beta^{\prime}}^{\mathrm{d}}\left(\mathbf{r}^{\prime}\right)\right]^{*} \\
\times \psi_{\alpha}^{\dagger}(\mathbf{x}) \psi_{\beta}^{\dagger}(\mathbf{y}) \psi_{\beta^{\prime}}\left(\mathbf{y}^{\prime}\right) \psi_{\alpha^{\prime}}\left(\mathbf{x}^{\prime}\right) .
\end{gathered}
$$

Here the single-particle coordinates are given in terms of the centre of mass and relative positions by $\mathbf{x}=\mathbf{R}+\mathbf{r} / 2, \mathbf{y}=$ $\mathbf{R}-\mathbf{r} / 2, \mathbf{x}^{\prime}=\mathbf{R}+\mathbf{r}^{\prime} / 2$, and $\mathbf{y}^{\prime}=\mathbf{R}-\mathbf{r}^{\prime} / 2$. 
The approach of Eq. (160) was introduced in the context of simulations of the Ramsey fringes of Fig. 2 (Köhler et al., $2003 \mathrm{a}$ b) and subsequently applied to the molecule production via magnetic field sweeps in Bose (Góral et al., 2004) and Fermi gases (Perali et al., 2005). In accordance with Eq. (160), the expectation value of Eq. (158) depends on the two-body correlation function,

$$
G_{\alpha^{\prime} \beta^{\prime}, \alpha \beta}^{(2)}\left(\mathbf{x}^{\prime}, \mathbf{y}^{\prime} ; \mathbf{x}, \mathbf{y}\right)=\left\langle\psi_{\alpha}^{\dagger}(\mathbf{x}) \psi_{\beta}^{\dagger}(\mathbf{y}) \psi_{\beta^{\prime}}\left(\mathbf{y}^{\prime}\right) \psi_{\alpha^{\prime}}\left(\mathbf{x}^{\prime}\right)\right\rangle .
$$

Here the average refers to the final state of the gas, $\rho_{N \mathrm{~B}}\left(t_{\mathrm{f}}\right)$. In the context of the dimer production via fast magnetic field sweeps of Subsection IV.C for instance, the dynamics of $G^{(2)}$ is determined simply by the two-body evolution operator, $U_{2 \mathrm{~B}}\left(t_{\mathrm{f}}, t_{\mathrm{i}}\right)$, in the limit of short interaction times. Given an ideal gas initial state, $\rho_{N \mathrm{~B}}\left(t_{\mathrm{i}}\right)$, associated with either Bose or Fermi atoms, the exact form of the initial two-body correlation function can be inferred from Wick's theorem of statistical mechanics (Fetter and Walecka, 1971; Matsubara, 1955; Wick, 1950). In the single resonance approach, the diatomic channel wave functions of the Feshbach molecular state occurring in Eq. (160) are determined in terms of the entranceand closed-channel components by the following formula:

$$
\sum_{\alpha, \beta}|\alpha, \beta\rangle \phi_{\alpha \beta}^{\mathrm{b}}(\mathbf{r})=|\mathrm{bg}\rangle \phi_{\mathrm{b}}^{\mathrm{bg}}(\mathbf{r})+|\mathrm{cl}\rangle \phi_{\mathrm{b}}^{\mathrm{cl}}(\mathbf{r})
$$

Based on these assumptions, the number of Feshbach molecules predicted by Eq. (160) strictly confirms the fast sweep limits of Eqs. (144), (131) and (145), in particular, their coefficients associated with the identical nature of the atoms. Besides these statistical estimates, the general observable of Eq. (160) may be applied to a variety of dynamical magnetic field variations as well as physical quantities. Given the associated two-body correlation function, it recovers, for instance, not only the measured maximum conversion into bound dimers of about $16 \%$ in Fig. 2 but also the populations of correlated pairs in continuum levels which constitute the burst of atoms (Köhler et al., 2003a). It turns out that due to the nonlinear magnetic field variation of Fig. 24, the determination of the time dependence of $G^{(2)}$ requires a description via techniques beyond the two-level mean field approach of Subsection IV.B.

\section{Dynamics of partially condensed Bose gases}

The precise many-body dynamics underlying all observations of Fig. 2 can be derived from the time dependent Schrödinger equation determined by the general Hamiltonian (Fetter and Walecka, 1971):

$$
\begin{aligned}
H= & \sum_{\alpha} \int d \mathbf{x} \psi_{\alpha}^{\dagger}(\mathbf{x})\left[-\frac{\hbar^{2}}{2 m} \nabla^{2}+E_{\alpha}^{\mathrm{a}}+V_{\alpha}^{\mathrm{ho}}(\mathbf{x})\right] \psi_{\alpha}(\mathbf{x}) \\
& +H_{\text {int. }}
\end{aligned}
$$

Here $E_{\alpha}^{\mathrm{a}}$ refers to an atomic Zeeman energy whose typical magnetic field dependence is illustrated in Fig. 17] for the example of ${ }^{87} \mathrm{Rb}$, and $V_{\alpha}^{\text {ho }}(\mathbf{x})$ describes the harmonic confinement due to an atom trap. In the context of dilute gases, the inter-atomic interactions are predominantly pairwise which implies the following potential energy contribution:

$$
\begin{array}{r}
H_{\text {int }}=\frac{1}{2} \sum_{\alpha, \beta, \alpha^{\prime}, \beta^{\prime}} \int d \mathbf{x} \int d \mathbf{y} V_{\alpha \beta, \alpha^{\prime} \beta^{\prime}}(\mathbf{x}-\mathbf{y}) \\
\times \psi_{\alpha}^{\dagger}(\mathbf{x}) \psi_{\beta}^{\dagger}(\mathbf{y}) \psi_{\beta^{\prime}}(\mathbf{y}) \psi_{\alpha^{\prime}}(\mathbf{x}) .
\end{array}
$$

Here $V_{\alpha \beta, \alpha^{\prime} \beta^{\prime}}(\mathbf{x}-\mathbf{y})$ denotes the two-body potential associated with the incoming and outgoing spin channels $\left|\alpha^{\prime}, \beta^{\prime}\right\rangle$ and $|\alpha, \beta\rangle$, respectively. We note that Eqs. (163) and (164) are formulated sufficiently generally to treat the inter-atomic interactions on a microscopic level, similarly to the coupled channels theory of Subsection $\amalg$ II.A As a consequence, the atomic Zeeman levels, $E_{\alpha}^{\mathrm{a}}$, appear separately in Eq. (163), while in the two-body Hamiltonian of Eq. (16) the dissociation threshold energy associated with the closed channel is included in the potential $V_{\mathrm{cl}}(B, r)$. Accordingly, all interactions, $V_{\alpha \beta, \alpha^{\prime} \beta^{\prime}}(\mathbf{x}-\mathbf{y})$, vanish in the limit of infinite distances, $r=|\mathbf{x}-\mathbf{y}| \rightarrow \infty$, similarly to the interaction matrix $V_{\text {int }}(r)$ of Eq. (11).

\section{Beyond mean field approaches}

Several techniques were employed in treatments of the many-body dynamics of dilute gases beyond the GrossPitaevskii and two-level mean field approaches of Subsection IV.B. These methods involve the Schwinger-Keldysh formalism (Keldysh, 1965; Schwinger, 1961) suitable for descriptions of phenomena associated with the evolution toward thermal equilibrium, such as the dimer production via adiabatic magnetic field sweeps of Fig. 22 (Williams et al., 2004a b, 2006). Practical approaches describing equilibration on even longer time scales, i.e. beyond the range of validity of the generalised Boltzmann equations derived from the Schwinger-Keldysh theory, may be based on the two-particle irreducible action (Baym, 1962; Cornwall et al., 1974; Luttinger and Ward, 1960). In the context of the dynamics of cold gases, such techniques have been implemented, to date, using the contact pseudo interaction in a single spatial dimension (Gasenzer et al., 2005; Rey et al., 2005). While the Ramsey fringes of Fig. 2 are sensitive to parameters of the inter-atomic potential besides the scattering length, the associated typical pulse sequence of Fig. 24 involves time scales sufficiently short for equilibration phenomena to be negligible. This implies that the dynamics of the gas is captured by extensions of mean field theory which account for the two-body time evolution beyond the Markov approximation (Holland et al., 2001; Köhler and Burnett, 2002; Naidon and Masnou-Seeuws, 2006; (Proukakis et al., 1998).

The usual quantities of interest in these short time quantum kinetic approaches may be expressed in terms of correlation functions, i.e. expectation values of normal ordered products of field operators, such as $G^{(2)}$ of Eq. (161). Their general expression reads $\left\langle\psi_{\beta}^{\dagger}(\mathbf{y}) \cdots \psi_{\alpha}(\mathbf{x})\right\rangle_{t}$ where all creation operators appear to the left of all annihilation operators, and the average refers to the many-body state at time $t$. The number of field operators constituting the product shall be referred to in 
the following as the order of the correlation function. Based on the Schrödinger equation, the associated dynamics is determined by

$$
i \hbar \frac{\partial}{\partial t}\left\langle\psi_{\beta}^{\dagger}(\mathbf{y}) \cdots \psi_{\alpha}(\mathbf{x})\right\rangle_{t}=\left\langle\left[\psi_{\beta}^{\dagger}(\mathbf{y}) \cdots \psi_{\alpha}(\mathbf{x}), H\right]\right\rangle_{t} .
$$

Here $H$ is the Hamiltonian of Eq. 163), and the symbol $[A, B]=A B-B A$ indicates the commutator of the operators $A$ and $B$. Due to the potential energy contribution of Eq. (164), the commutator on the right hand side of Eq. (165) gives rise to products containing two more field operators than the expectation value to the left. This implies that the dynamics of any one correlation function is determined by a coupled set of equations involving all the others. Approximate solutions to Eq. [165), therefore, often rely upon schemes for the truncation of the associated infinite hierarchy of dynamical equations. The range of applicability of such approaches is sensitive to the initial state of the many-body system.

In the context of weakly interacting gases close to thermal equilibrium, an approximate form of all correlation functions may be determined using Wick's theorem of statistical mechanics (Fetter and Walecka, 1971; Matsubara, 1955; Wick, 1950). To this end, it is instructive to introduce the connected correlation functions (Weinberg, 1996), sometimes referred to as cumulants. These quantities may be inferred recursively from a decomposition of each correlation function into a sum of all possible products of cumulants which preserve the order of appearance of the operators. Given a set of Bose field operators, $A, B$ and $C$, for instance, the first three cumulants, denoted by $\langle A\rangle^{\mathrm{c}},\langle B A\rangle^{\mathrm{c}}$ and $\langle C B A\rangle^{\mathrm{c}}$, are determined implicitly by the following relations:

$$
\begin{aligned}
\langle A\rangle= & \langle A\rangle^{\mathrm{c}}, \\
\langle B A\rangle= & \langle B A\rangle^{\mathrm{c}}+\langle A\rangle^{\mathrm{c}}\langle B\rangle^{\mathrm{c}}, \\
\langle C B A\rangle= & \langle C B A\rangle^{\mathrm{c}}+\langle B A\rangle^{\mathrm{c}}\langle C\rangle^{\mathrm{c}}+\langle C A\rangle^{\mathrm{c}}\langle B\rangle^{\mathrm{c}} \\
& +\langle C B\rangle^{\mathrm{c}}\langle A\rangle^{\mathrm{c}}+\langle A\rangle^{\mathrm{c}}\langle B\rangle^{\mathrm{c}}\langle C\rangle^{\mathrm{c}} .
\end{aligned}
$$

The cumulants associated with Fermi field operators may be inferred from expressions similar to Eqs. (166), (167) and (168). In accordance with the anti-commutation relation of Eq. (159), however, the number of commutations of field operators in the decompositions needs to be accounted for by an appropriate sign of each contribution. In both cases, Bose and Fermi atoms, Wick's theorem reduces to the statement that all cumulants containing more than two field operators vanish provided that the gas is ideal and in thermal equilibrium. The magnitude of higher order cumulants therefore provides a measure of the deviations of the state from the grand canonical density matrix in the absence of inter-atomic interactions.

This suggests that in the context of dilute, weakly interacting gases a reasonable quantum kinetic approach may be based on the transformation of Eq. (165) into the associated set of dynamical equations for cumulants. In accordance with Wick's theorem, this coupled system allows for an approximate truncation at any order of correlation usually determined by external driving fields as well as the initial state (Fricke, 1996). The dilute gas of ${ }^{85} \mathrm{Rb}$ atoms in the Ramsey interferometry experiments illustrated in Fig. 25 was prepared as a
Bose-Einstein condensate in the $\left(f=2, m_{f}=-2\right)$ Zeeman level. Such a coherent initial state gives rise to a mean field, $\Psi_{\alpha}(\mathbf{x}, t)=\left\langle\psi_{\alpha}(\mathbf{x})\right\rangle_{t}$, describing the density associated with the macroscopically occupied mode via its modulus squared (Dalfovo et al., 1999). In accordance with Fig. 2, the condensate is depleted due to the near resonant magnetic field variation of Fig. 24 This implies that, in addition to the mean field, a minimum set of cumulants describing this experiment is given, respectively, by the pair function and the one-body density matrix of the non-condensed component:

$$
\begin{aligned}
& \Phi_{\alpha \beta}(\mathbf{x}, \mathbf{y}, t)=\left\langle\psi_{\beta}(\mathbf{y}) \psi_{\alpha}(\mathbf{x})\right\rangle_{t}-\Psi_{\alpha}(\mathbf{x}, t) \Psi_{\beta}(\mathbf{y}, t), \\
& \Gamma_{\alpha \beta}(\mathbf{x}, \mathbf{y}, t)=\left\langle\psi_{\beta}^{\dagger}(\mathbf{y}) \psi_{\alpha}(\mathbf{x})\right\rangle_{t}-\Psi_{\alpha}(\mathbf{x}, t) \Psi_{\beta}^{*}(\mathbf{y}, t) .
\end{aligned}
$$

Accordingly, at any time, $t$, the density of atoms in the Zeeman state with the index $\alpha$ is given by $\left|\Psi_{\alpha}(\mathbf{x}, t)\right|^{2}+\Gamma_{\alpha \alpha}(\mathbf{x}, \mathbf{x}, t)$. Due to the weak interactions of the gas at the beginning of the magnetic field pulse sequence, the second order cumulants of Eqs. (169) and (170) are negligible initially, i.e. $\Phi_{\alpha \beta}\left(\mathbf{x}, \mathbf{y}, t_{\mathrm{i}}\right)=$ $0=\Gamma_{\alpha \beta}\left(\mathbf{x}, \mathbf{y}, t_{\mathrm{i}}\right)$.

To a first approximation, the dynamical equations (165) may be transformed and truncated in such a way that they just include products of normal ordered cumulants containing at most three field operators (Köhler and Burnett, 2002). This yields the following relation for the time derivative of the condensate mean field:

$$
\begin{aligned}
i \hbar \dot{\Psi}_{\alpha}(\mathbf{x}, t)=H_{\alpha}^{1 \mathrm{~B}} \Psi_{\alpha}(\mathbf{x}, t)+ & \sum_{\alpha, \alpha^{\prime}, \beta^{\prime}} \int d \mathbf{y} V_{\alpha \beta, \alpha^{\prime} \beta^{\prime}}(\mathbf{r}) \\
& \times \Psi_{\beta}^{*}(\mathbf{y}, t)\left\langle\psi_{\beta^{\prime}}(\mathbf{y}) \psi_{\alpha^{\prime}}(\mathbf{x})\right\rangle_{t} .
\end{aligned}
$$

Here $\mathbf{r}=\mathbf{x}-\mathbf{y}$ refers to the relative coordinates of a pair of atoms at the positions $\mathbf{x}$ and $\mathbf{y}$, and the one-body Hamiltonian, $H_{\alpha}^{1 \mathrm{~B}}$, consists of the kinetic and Zeeman energies as well as the trap potential of Eq. (163). The correlation function, $\left\langle\psi_{\beta^{\prime}}(\mathbf{y}) \psi_{\alpha^{\prime}}(\mathbf{x})\right\rangle_{t}$, on the right hand side of Eq. (171) is determined in terms of cumulants by Eq. (169). In addition to Eq. (171), the dynamical equation associated with the pair function reads:

$$
\begin{aligned}
i \hbar \dot{\Phi}_{\alpha \beta}(\mathbf{x}, \mathbf{y}, t)= & \sum_{\alpha^{\prime}, \beta^{\prime}}\left[H_{\alpha \beta, \alpha^{\prime} \beta^{\prime}}^{2 \mathrm{~B}} \Phi_{\alpha^{\prime} \beta^{\prime}}(\mathbf{x}, \mathbf{y}, t)\right. \\
& \left.+V_{\alpha \beta, \alpha^{\prime} \beta^{\prime}}(\mathbf{r}) \Psi_{\alpha^{\prime}}(\mathbf{x}, t) \Psi_{\beta^{\prime}}(\mathbf{y}, t)\right] .
\end{aligned}
$$

Here $H_{\alpha \beta, \alpha^{\prime} \beta^{\prime}}^{2 \mathrm{~B}}$ denotes the two-body Hamiltonian matrix associated with the incoming and outgoing spin channels $\left|\alpha^{\prime}, \beta^{\prime}\right\rangle$ and $|\alpha, \beta\rangle$, respectively, describing both the centre of mass and relative motions of an atom pair.

Given this first order truncation scheme, Eqs. (171) and (172) uniquely determine the condensate mean field as well as the pair function. It turns out that the dynamical equation associated with the one-body density matrix of the noncondensed component may be solved implicitly in terms of the pair function. This yields:

$$
\Gamma_{\alpha \beta}(\mathbf{x}, \mathbf{y}, t)=\sum_{\gamma} \int d \mathbf{z} \Phi_{\alpha \gamma}(\mathbf{x}, \mathbf{z}, t)\left[\Phi_{\beta \gamma}(\mathbf{y}, \mathbf{z}, t)\right]^{*} .
$$


In accordance with the general expression for the associated observable, $\mathrm{N}=\sum_{\alpha} \int d \mathbf{x} \psi_{\alpha}^{\dagger}(\mathbf{x}) \psi_{\alpha}(\mathbf{x})$, the expectation value of the number of atoms, $N$, is strictly conserved by Eqs. (171), (172) and (173) at all times, i.e.

$$
\sum_{\alpha} \int d \mathbf{x}\left[\left|\Psi_{\alpha}(\mathbf{x}, t)\right|^{2}+\Gamma_{\alpha \alpha}(\mathbf{x}, \mathbf{x}, t)\right]=N
$$

In addition, Eqs. (170) and (173) preserve the positivity of the one-body density matrix $\left\langle\psi_{\beta}^{\dagger}(\mathbf{y}) \psi_{\alpha}(\mathbf{x})\right\rangle_{t}$, a consequence of the unitary time evolution, which is not necessarily recovered by higher order approximation schemes.

In the context of dimer formation in partially condensed Bose gases, similar extensions of mean field theory are based on the Hartree-Fock Bogoliubov approach (Holland et al., 2001) as well as the reduced pair wave function approximation (Cherny and Shanenko, 2000; Naidon and Masnou-Seeuws, 2003, 2006). As these methods all originate from Eq. (165), their short time asymptotic limits agree with the exact result given by the perturbation expansion of the dynamical many-body Schrödinger equation. In addition, the correlation functions predicted by any one of these approaches (Holland et al., 2001; Köhler and Burnett, 2002; Naidon and Masnou-Seeuws, 2003, 2006) are free of secular long time asymptotic behaviour, a common artifact of perturbation theory associated with a spurious polynomial time dependence. Both the cumulant (Köhler and Burnett, 2002) and the reduced pair wave function approach (Naidon and Masnou-Seeuws, 2003, 2006) were formulated in such a way that they are compatible with the use of microscopic potentials beyond contact pseudo interactions.

Most implementations of quantum kinetic approaches to the dimer production via Feshbach resonances to date are based on single-channel or two-channel single resonance binary interactions illustrated in Subsection $\amalg$.B. This implies that the Bose-Einstein condensate mode is described by a single mean field, $\Psi(\mathbf{x}, t)$, associated with the Zeeman level in which the gas is prepared. In addition, the entrance- and closed-channel components of the pair function may be inferred, similarly to Eq. 162, from the following formula:

$$
\sum_{\alpha, \beta}|\alpha, \beta\rangle \Phi_{\alpha \beta}(\mathbf{x}, \mathbf{y}, t)=|\mathrm{bg}\rangle \Phi_{\mathrm{bg}}(\mathbf{x}, \mathbf{y}, t)+|\mathrm{cl}\rangle \Phi_{\mathrm{cl}}(\mathbf{x}, \mathbf{y}, t)
$$

The resonance mean field of Subsection IV.B is determined by the relation $\Phi_{\mathrm{cl}}(\mathbf{x}, \mathbf{y}, t)=\Psi_{\text {res }}(\mathbf{R}, t) \phi_{\text {res }}(r)$. Here $\mathbf{R}=$ $(\mathbf{x}+\mathbf{y}) / 2$ refers to the centre of mass coordinates of a pair of atoms and $r=|\mathbf{x}-\mathbf{y}|$ denotes their relative distance. Based on a two-channel implementation of Eqs. (171) and (172), the two-level mean field approach (Drummond et al., 1998; Timmermans et al., 1998, 1999a; Tommasini et al., 1998) may be recovered by formally solving the dynamical equation associated with the entrance-channel component of the pair function. A subsequent elimination of $\Phi_{\mathrm{bg}}(\mathbf{x}, \mathbf{y}, t)$ from the coupled equations (171) and (172) yields the functional form of the right hand sides of Eqs. (136) and (137). The coupling constants of Eq. (134) and of the entrance channel contact pseudo interaction are determined by the Markov approximation (Góral et al., 2004).

We note that truncation schemes of higher order than Eqs. (171) and (172) can involve cross coupling terms that give rise to pair functions beyond the two-channel decomposition of Eq. (175). Such a scenario occurs in quantum kinetic approaches associated with both Bose and Fermi gases provided that one and the same atomic Zeeman state is shared between the two-body entrance and closed channels (Bruun et al., 2005; Parish et al., 2005). This, in turn, restricts the applicability of commonly employed model Hamiltonians (Friedberg and Lee, 1989; Ranninger and Robaszkiewicz, 1985) which separate out the resonance state in the description of Feshbach molecule production.

\section{The remnant Bose-Einstein condensate}

The dynamics of the condensate mean field associated with the Ramsey interferometry experiments (Donley et al., 2002) was described using the Hartee-Fock Bogoliubov method (Kokkelmans and Holland, 2002) and related techniques (Mackie et al., 2002b), as well as the first order cumulant approach of Eqs. (171) and (172) (Köhler et al., 2003a). Such studies involving beyond mean field theories, can often be simplified by eliminating the pair function from the set of dynamical or associated eigenvalue equations, respectively (Burnett, 1999). In the context of Eqs. (171) and (172), this procedure yields (Köhler and Burnett, 2002):

$$
\begin{aligned}
i \hbar \dot{\Psi}(\mathbf{x}, t)= & H_{1 \mathrm{~B}} \Psi(\mathbf{x}, t) \\
& -\Psi^{*}(\mathbf{x}, t) \int_{t_{\mathrm{i}}}^{\infty} d t^{\prime} \Psi^{2}\left(\mathbf{x}, t^{\prime}\right) \frac{\partial}{\partial t^{\prime}} h\left(t, t^{\prime}\right) .
\end{aligned}
$$

Here $H_{1 \mathrm{~B}}$ is the one-body Hamiltonian associated with the initial Zeeman state of the condensed atoms. The coupling function on the right hand side of Eq. (176),

$$
h\left(t, t^{\prime}\right)=\theta\left(t-t^{\prime}\right)(2 \pi \hbar)^{3}\left\langle 0, \mathrm{bg}\left|V U_{2 \mathrm{~B}}\left(t, t^{\prime}\right)\right| 0, \mathrm{bg}\right\rangle
$$

is determined by the two-body time evolution operator of Eq. (150). Here $\langle\mathbf{r} \mid 0\rangle=1 /(2 \pi \hbar)^{3 / 2}$ denotes the zero momentum plane wave of the relative motion of an atom pair and $V$ is the microscopic potential matrix of Eq. (164).

The representation of Eqs. (176) and (177) shows that all findings of Subsection V.A about the two-body time evolution and physical origin of the Ramsey fringes are included in the quantum kinetic approach. Its degree of accuracy in comparison to experimental data of the condensate component remaining at the end of a magnetic field pulse sequence (Claussen et al.,2003) is illustrated in the inset of Fig. 25. The associated theory curve is based on an implementation of the two-channel singe-resonance approach of Subsection III.Gusing a separable background scattering potential (Góral et al., 2005). 


\section{Feshbach molecule and burst components}

In accordance with Eq. (174), the total number of atoms can be decomposed into a mean field contribution as well as a noncondensed component, $N_{\mathrm{nc}}(t)$, described by the density matrix of Eq. (170). The physical significance of $N_{\text {nc }}(t)$ in the Ramsey interferometry experiments (Donley et al., 2002) may be inferred from Eq. (173). To this end, it is instructive to replace the spatial average of Eq. (173) using the completeness of the set of dressed bound and continuum energy states associated with the magnetic field strength at time $t$. This leads to the following relation:

$$
\begin{aligned}
N_{\mathrm{nc}}(t)=\int d \mathbf{R}\left[\int d \mathbf{p}\left|\left\langle\mathbf{R}, \phi_{\mathbf{p}} \mid \Phi(t)\right\rangle\right|^{2}\right. \\
\left.+\left|\left\langle\mathbf{R}, \phi_{\mathrm{b}} \mid \Phi(t)\right\rangle\right|^{2}\right] .
\end{aligned}
$$

Here $\mathbf{R}$ may be interpreted in terms of the centre of mass position of an atom pair, and $|\Phi(t)\rangle$ refers to the pair function whose channel components in the spatial representation are given by Eq. (169). For simplicity, the spectral decomposition of Eq. (178) includes just the dressed continuum states, $\left|\phi_{\mathbf{p}}\right\rangle$, and the Feshbach molecular state, $\left|\phi_{\mathrm{b}}\right\rangle$. More deeply bound levels are neglected.

Equation (178) suggests that the continuum contribution may be interpreted in terms of correlated atom pairs associated with relative momenta, $\mathbf{p}$, while the bound state part yields the number of atoms converted into dimers. An analysis based on the observable of Eq. (160) confirms this view (Köhler et al., 2003a). Its practical implementation based on Eqs. (171) and (172) involves a cumulant expansion of the two-body correlation function in accordance with the first order truncation scheme. This yields:

$$
\begin{aligned}
G_{\alpha^{\prime} \beta^{\prime}, \alpha \beta}^{(2)}\left(\mathbf{x}^{\prime}, \mathbf{y}^{\prime} ; \mathbf{x}, \mathbf{y} ; t\right)= & \left\langle\psi_{\alpha}^{\dagger}(\mathbf{x}) \psi_{\beta}^{\dagger}(\mathbf{y})\right\rangle_{t} \\
& \times\left\langle\psi_{\beta^{\prime}}\left(\mathbf{y}^{\prime}\right) \psi_{\alpha^{\prime}}\left(\mathbf{x}^{\prime}\right)\right\rangle_{t} .
\end{aligned}
$$

Here the correlation functions on the right hand side can be expressed in terms of pair functions and condensate mean fields via Eq. (169). Using Eqs. (162) and (179), it turns out that the diatomic number operator of Eq. (160) gives rise to a dimer mean field,

$$
\begin{aligned}
\Psi_{\mathrm{b}}(\mathbf{R}, t)= & \frac{1}{\sqrt{2}}\left\{\left\langle\mathbf{R}, \phi_{\mathrm{b}} \mid \Phi(t)\right\rangle\right. \\
& \left.+\int d \mathbf{r}\left[\phi_{\mathrm{b}}^{\mathrm{bg}}(\mathbf{r})\right]^{*} \Psi(\mathbf{x}, t) \Psi(\mathbf{y}, t)\right\},
\end{aligned}
$$

which determines the number of Feshbach molecules produced via the relation $N_{\mathrm{d}}(t)=\int d \mathbf{R}\left|\Psi_{\mathrm{b}}(\mathbf{R}, t)\right|^{2}$.

The first term on the right hand side of Eq. (180) recovers the bound state contribution of Eq. (178). In accordance with the long range of the ${ }^{85} \mathrm{Rb}_{2}$ entrance-channel wave functions of Fig. 11 the second term may be interpreted in terms of the overlap between the dimers produced and the surrounding gas. Its magnitude is determined by the dilute gas parameter squared, $n_{\mathrm{c}}(t) a^{3}$, where $n_{\mathrm{c}}(t)$ is the average condensate density and $a$ refers to the scattering length associated with the magnetic field strength at time $t$ (Köhler et al., 2003b). Consequently, the existence of this overlap term reflects the breakdown of the concept of diatomic molecules in the environment of a strongly interacting gas. This breakdown occurs when the bond length $\langle r\rangle=a / 2$ of the universal dimer wave function of Eq. (2) is comparable to the mean inter-atomic distance $n_{\mathrm{c}}^{-1 / 3}$ of the condensate. Similar phenomena are visible in Figures 13,14 and 15 .

At the end of the magnetic field pulse sequence of Fig. 24 the gas is weakly interacting and the second, overlap term on the right hand side of Eq. (180) is negligible. Based on Eqs. (176) and (180), the predicted number of atoms converted into dimers recovers the $16 \%$ maximum fraction of missing atoms of Fig. 2 as well as its modulation as a function of $t_{\text {evolve }}$ (Köhler et al., 2003a). In accordance with Fig. 15. the fact that these Feshbach molecules were not detected reflects their lifetime with respect to spin relaxation on the order of only $100 \mu \mathrm{s}$ at the final magnetic field strength of about $162 \mathrm{G}$ (Köhler et al., 2005; Thompson et al., 2005b). An analysis based on Eqs. (160) and (179) also shows that the continuum contribution on the right hand side of Eq. (178) is associated with correlated atom pairs which constitute the measured burst component of Fig. 2] (Köhler et al., 2003a).

\section{Three-component Ramsey fringes}

The physical origin of the Ramsey fringes (Donley et al., 2002) was the subject of several theoretical works (Góral et al., 2005; Köhler et al., 2003a; Kokkelmans and Holland, 2002; Mackie et al., 2002b) using different beyond mean field approaches. Figure 26 shows such a prediction (Góral et al., 2005) referring to the magnetic field pulse sequence and number of atoms reported for the measurements of Fig. 2. The overall picture indicates that the quantum kinetic approaches support the interpretation of Subsection V.A provided that the gas is weakly interacting during the evolution period.

Some measurements were performed also in the regime of near resonant evolution fields, $B_{\text {evolve }}<157 \mathrm{G}$. Under these conditions, the scattering length as well as the spatial extent of the Feshbach molecule are comparable to the mean interatomic distance of the gas. As the atomic and dimer components are no longer orthogonal during the evolution period, the classic concept of a Ramsey interferometer is expected to

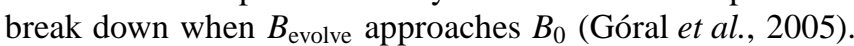
Such a phenomenon was observed in terms of a pronounced damping of the fringe pattern accompanied by an upward shift of its frequency with respect to the two-body prediction of $\left|E_{\mathrm{b}}^{\text {evolve }}\right| / h$ (Claussen et al., 2003). This shift was subsequently attributed to genuinely many-particle corrections to the intuitive viewpoint illustrated in Fig. 25 (Duine and Stoof, 2003b; Góral et al., 2005). 


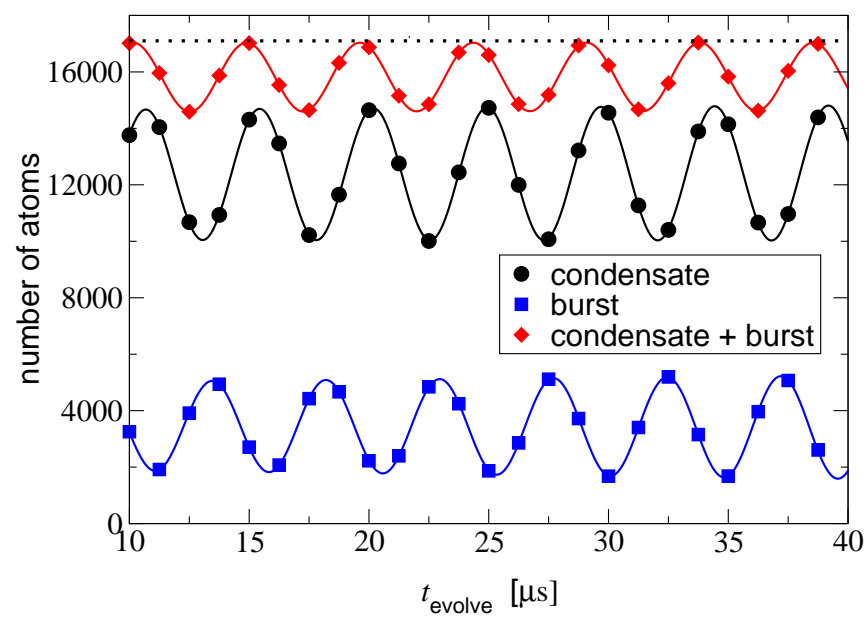

FIG. 26 (Colour in online edition) Predicted Ramsey fringes between three components of a dilute gas of ${ }^{85} \mathrm{Rb}$ (Góral et al., 2005) at the end of the magnetic field pulse sequence of Fig. 24 versus the evolution time, $t_{\text {evolve }}$. The circles refer to the remnant Bose-Einstein condensate, while the squares indicate the final population of correlated atom pairs constituting the burst component. The remnant condensate and burst add up to the number of detectable atoms (diamonds). Its difference to the total number of $N=17100$ atoms (dotted line) in the experiments (Donley et al., 2002) determines the population of Feshbach molecules. The magnetic field strength in the evolution period of $B_{\text {evolve }}=159.84 \mathrm{G}$ associated with these predictions refers to the conditions of the measured fringes of Fig.2

\section{CONCLUSIONS AND OUTLOOK}

This article has given an overview of a variety of concepts associated with the description of properties of Feshbach molecules as well as the techniques for their production in the environment of a cold atomic gas. Its conclusions can be summarised as follows: Coupled channels theory provides the foundation for an accurate understanding of the diatomic molecular and collision physics. Its predictions can be recovered, in an experimentally relevant energy range about the dissociation threshold, by two-channel approaches characterised in terms of just a few measurable quantities. These involve the resonance position and width, the background scattering length and the van der Waals dispersion coefficient, as well as the difference in the magnetic moments associated with the resonance state and a pair of asymptotically separated atoms. Universal properties of Feshbach molecules and the low energy collision physics are directly related to Wigner's threshold law. Such concepts associated with twobody physics treat identical bosons and fermions in different Zeeman states as well as distinguishable atoms in essentially the same manner. The fundamental transition amplitudes for the Feshbach molecular association of an atom pair via linear magnetic field sweeps can be represented analytically, in the context of two-channel single-resonance approaches. Such an exact treatment provides the foundation of the Landau-Zener approach to the dimer production in tight traps, such as optical lattice sites, as well as in cold gases in the fast sweep limit. While these limits including their quantum statistical phenomena can be understood largely in terms of classical probability theory, the saturation of molecule production involves the many-body dynamics of thermalisation. Given sufficiently short time scales, beyond mean field approaches can provide quantitative descriptions of Feshbach molecule production in Bose-Einstein condensates also in the context of non-linear magnetic field variations.

Closely related to the enhancement of the $s$-wave scattering cross sections discussed in this review are similar magnetically tunable Feshbach resonance phenomena involving finite angular momentum quantum numbers, $\ell>0$. Associated scattering amplitudes, $f_{\ell}(p)$, are usually negligible compared to the $s$-wave contribution in cold collision physics due to their $p^{2 \ell}$ scaling with the relative momentum of an atom pair. In gases of identical Fermi atoms involving just a single Zeeman state, however, only the odd partial waves contribute to diatomic scattering, in accordance with the Pauli exclusion principle. In such cases, $p$-wave $(\ell=1)$ resonances have been observed in gases of both ${ }^{40} \mathrm{~K}$ (Regal et al., 2003b) and ${ }^{6} \mathrm{Li}$ (Schunck et al., 2005; Zhang et al., 2004), providing possibilities of magnetically tuning interactions as well as associating dimers.

Optical Feshbach resonances (Bohn and Julienne, 1997, 1999; Fedichev et al., 1996) provide an alternative approach to tune $s$-wave scattering cross sections in cold gases. This technique relies upon coupling the internal states of a pair of separated atoms to a closed channel using laser light instead of a homogeneous magnetic field. According to experimental studies in cold gases of ${ }^{23} \mathrm{Na}$ (Fatemi et al., 2000) and ${ }^{87} \mathrm{Rb}$ Bose-Einstein condensates (Theis et al., 2004), significant changes of the scattering length tend to cause substantial atom loss due to spontaneous photon emission. Recent predictions indicate, however, that such loss may be efficiently suppressed in applications to cold gases of alkaline earth metal atoms, such as bosonic calcium, as well as ytterbium (Ciurylo et al., 2005). As these atoms lack hyperfine structure due to the absence of nuclear spin, magnetic Feshbach resonances are ruled out. Their optical counterpart may therefore provide the only possibility of tuning interactions in such gases.

Inter-species magnetic Feshbach resonances were observed in boson-fermion mixtures consisting of cold gases of ${ }^{6} \mathrm{Li}$ and ${ }^{23} \mathrm{Na}$ (Stan et al., 2004) as well as ${ }^{40} \mathrm{~K}$ and ${ }^{87} \mathrm{Rb}$ (Inouye et al., 2004). Their binary physics can be described using the approaches outlined in this review, whereas the collisional stability of inter-species Feshbach molecules requires special attention (Petrov et al., 2005a). The production of such diatomic molecules is of particular interest because of a possibly polar character of their bonds (Doyle et al., 2004). Cold polar molecules composed of Bose atoms have been produced via photo-association (Kerman et al., 2004; Wang et al., 2004). According to predictions (Baranov et al., 2002), the long range, anisotropy and magnitude of the dipole-dipole interactions give rise to a host of new features in the associated fermionic and bosonic superfuids. Arrays of polar molecules in optical lattices may provide an opportunity for studies of super-solid phases (Góral et al., 2002). It is hoped that heavy polar molecules will enable us to improve tests of fundamental 
physical symmetries, including measurements of the electron dipole moment (Hudson et al., 2002; Sandars, 1975). Stabilisation with respect to collisional relaxation of excited molecular states may be achieved via transfer to their vibrational ground states, an approach also demonstrated in the context of photo-association (Sage et al., 2005). Such a de-excitation, in turn, tends to enhance the polar character of their bonds.

Few-body scattering phenomena, such as dimer-dimer resonances recently observed in cold gases of ${ }^{133} \mathrm{Cs}_{2}$, Chin et al. 2005), may provide possibilities of extending existing techniques of Feshbach molecule production to more complex species. One of the long standing goals in this context is an experimental confirmation of predictions associated with Efimov's effect in the three-body energy spectrum of identical Bose particles (Efimov, 1970, 1971). The Efimov spectrum consists of an infinite sequence of isotropic three-body bound states accumulating at the dissociation threshold, which occurs in the limit of infinite $s$-wave scattering length of each two-body subsystem. This scenario is therefore directly related to the long range nature of weakly bound twoparticle halo states (Jensen et al., 2004) and may be realised via magnetic Feshbach resonances in cold gases. Signatures of the emergence of such Efimov states are predicted to occur in terms of a modulation of three-body recombination loss rates as a function of the near resonant magnetic field strength (Braaten and Hammer, 2001; Esry et al., 1999; Nielsen and Macek, 1999). Recently, the first experimental evidence for this phenomenon has been reported (Kraemer et al., 2006). Such inelastic scattering resonances may be exploited to associate meta-stable three-body Efimov molecules from cold Bose gases loaded into optical lattices, using the magnetic field sweep technique discussed in this review (Stoll and Köhler, 2005).

\section{Acknowledgements}

We are grateful to Johannes Hecker Denschlag, Stephan Dürr, Rudi Grimm, Tom Hanna, Eleanor Hodby, Randy Hulet, Debbie Jin, Wolfgang Ketterle, Servaas Kokkelmans, Tobias Kraemer, Takashi Mukaiyama, Christoph Nägerl, Nicolai Nygaard, Cindy Regal, Gregor Thalhammer, Sarah Thompson, Boudewijn Verhaar, Carl Wieman, Jamie Williams and Kaiwen $\mathrm{Xu}$ for providing their experimental and theoretical data to us. We thank Keith Burnett, Cheng Chin, Thomas Gasenzer, Josh Milstein, Bill Stwalley, Marzena Szymańska and Eite Tiesinga for valuable discussions. This review was supported by the Royal Society (T.K. and K.G.). P.S.J. gratefully acknowledges his support from the Office of Naval Research.

\section{References}

van Abeelen, F. A., and B. J. Verhaar, 1998, unpublished, quoted by S. Inouye et al. (Inouve et al., 1998).

van Abeelen, F. A., and B. J. Verhaar, 1999, Phys. Rev. Lett. 83, 1550.

Abraham, E. R. I., W. I. McAlexander, J. M. Gerton, R. G. Hulet, R. Côté, and A. Dalgarno, 1997, Phys. Rev. A 55, R3299.
Anderson, M. H., J. R. Ensher, M. R. Matthews, C. E. Wieman, and E. A. Cornell, 1995, Science 269, 5221.

Arimondo, E., M. Inguscio, and P. Violino, 1977, Rev. Mod. Phys. 49, 31 .

Balakrishnan, N., R. C. Forrey, and A. Dalgarno, 1997, Chem. Phys. Lett. 280, 1.

Baranov, M., L. Dobrek, K. Góral, L. Santos, and M. Lewenstein, 2002, Phys. Scr. T 102, 74.

Bartenstein, M., A. Altmeyer, S. Riedl, R. Geursen, S. Jochim, C. Chin, J. H. Denschlag, R. Grimm, A. Simoni, E. Tiesinga, C. J. Williams, and P. S. Julienne, 2005, Phys. Rev. Lett. 94, 103201.

Bartenstein, M., A. Altmeyer, S. Riedl, S. Jochim, C. Chin, J. H. Denschlag, and R. Grimm, 2004, Phys. Rev. Lett. 92, 120401.

Baym, G., 1962, Phys. Rev. 127, 1391.

Bender, P. L., 1963, Phys. Rev. 132, 2154.

Bethe, H. A., 1949, Phys. Rev. 76, 38.

Blatt, J. M., and V. F. Weisskopf, 1952, Theoretical Nuclear Physics (Wiley, New York).

Bloch, F., 1928, Z. Phys. 52, 555.

Blume, D., and C. H. Greene, 2002, Phys. Rev. A 65, 043613.

Bohn, J. L., and P. S. Julienne, 1997, Phys. Rev. A 56, 1486.

Bohn, J. L., and P. S. Julienne, 1999, Phys. Rev. A 60, 414.

Bolda, E. L., E. Tiesinga, and P. S. Julienne, 2002, Phys. Rev. A 66, 013403.

Borca, B., D. Blume, and C. H. Greene, 2003, New J. Phys. 5, 111.

Bose, S. N., 1924, Z. Phys. 26, 178.

Bourdel, T., L. Khaykovich, J. Cubizolles, J. Zhang, F. Chevy, M. Teichmann, L. Tarruell, S. J. J. M. F. Kokkelmans, and C. Salomon, 2004, Phys. Rev. Lett. 93, 050401.

Braaten, E., and H.-W. Hammer, 2001, Phys. Rev. Lett. 87, 160407.

Braaten, E., H.-W. Hammer, and M. Kusunoki, 2003, Comment on "Ramsey Fringes in a Bose-Einstein Condensate between Atoms and Molecules", e-print cond-mat/0301489.

Bradley, C. C., C. A. Sackett, and R. G. Hulet, 1997, Phys. Rev. Lett. 78, 985.

Bradley, C. C., C. A. Sackett, J. J. Tollett, and R. G. Hulet, 1995, Phys. Rev. Lett. 75, 1687.

Bransden, B. H., and C. J. Joachain, 2003, Physics of Atoms and Molecules (Pearson Education Ltd., Harlow).

Breit, G., and I. I. Rabi, 1931, Phys. Rev. 38, 2082.

Brouard, S., and J. Plata, 2005, Phys. Rev. A 72, 023620.

Bruun, G. M., A. D. Jackson, and E. E. Kolomeitsev, 2005, Phys. Rev. A 71, 052713.

Bruun, G. M., and C. J. Pethick, 2004, Phys. Rev. Lett. 92, 140404.

Burke, J. P., C. H. Greene, and J. L. Bohn, 1998, Phys. Rev. Lett. 81, 3355.

Burnett, K., 1999, in Bose-Einstein Condensation in Atomic Gases, edited by M. Inguscio, S. Stringari, and C. E. Wieman, International School of Physics Enrico Fermi (IOS Press, Amsterdam), p. 265.

Burt, E. A., R. W. Ghrist, C. J. Myatt, M. J. Holland, E. A. Cornell, and C. E. Wieman, 1997, Phys. Rev. Lett. 79, 337.

Busch, T., B.-G. Englert, K. Rzążewski, and M. Wilkens, 1998, Found. Phys. 28, 549.

Cherny, A. Y., and A. A. Shanenko, 2000, Phys. Rev. E 62, 1646.

Child, M. S., 1974, Molecular Collision Theory (Academic, London).

Chin, C., 2005, A simple model of Feshbach molecules, e-print condmat/0506313.

Chin, C., M. Bartenstein, A. Altmeyer, S. Riedl, S. Jochim, J. H. Denschlag, and R. Grimm, 2004a, Science 305, 1128.

Chin, C., and R. Grimm, 2004, Phys. Rev. A 69, 033612.

Chin, C., A. J. Kerman, V. Vuletic, and S. Chu, 2003, Phys. Rev. Lett. 90, 033201. 
Chin, C., T. Kraemer, M. Mark, J. Herbig, P. Waldburger, H.-C. Nägerl, and R. Grimm, 2005, Phys. Rev. Lett. 94, 123201.

Chin, C., V. Vuletic, A. J. Kerman, S. Chu, E. Tiesinga, P. J. Leo, and P. S. Julienne, 2004b, Phys. Rev. A 70, 032701.

Chwedeńczuk, J., K. Góral, T. Köhler, and P. S. Julienne, 2004, Phys. Rev. Lett. 93, 260403.

Ciurylo, R., E. Tiesinga, and P. S. Julienne, 2005, Phys. Rev. A 71, 030701(R).

Claussen, N. R., E. A. Donley, S. T. Thompson, and C. E. Wieman, 2002, Phys. Rev. Lett. 89, 010401.

Claussen, N. R., S. J. J. M. F. Kokkelmans, S. T. Thompson, E. A. Donley, E. Hodby, and C. E. Wieman, 2003, Phys. Rev. A 67, 060701(R).

Cornish, S. L., N. R. Claussen, J. L. Roberts, E. A. Cornell, and C. E. Wieman, 2000, Phys. Rev. Lett. 85, 1795.

Cornwall, J. M., R. Jackiw, and E. Tomboulis, 1974, Phys. Rev. D 10, 2428.

Courteille, P., R. S. Freeland, D. J. Heinzen, F. A. van Abeelen, and B. J. Verhaar, 1998, Phys. Rev. Lett. 81, 69.

Cubizolles, J., T. Bourdel, S. J. J. M. F. Kokkelmans, G. V. Shlyapnikov, and C. Salomon, 2003, Phys. Rev. Lett. 91, 240401.

Cvitas, M. T., P. Soldán, J. M. Hutson, P. Honvault, and J.-M. Launay, 2002, Phys. Rev. Lett. 89, 153201.

Cvitas, M. T., P. Soldán, J. M. Hutson, P. Honvault, and J.-M. Launay, 2005a, Phys. Rev. Lett. 94, 200402.

Cvitas, M. T., P. Soldán, J. M. Hutson, P. Honvault, and J.-M. Launay, 2005b, Phys. Rev. Lett. 94, 033201.

Dalfovo, F., S. Giorgini, L. P. Pitaevskii, and S. Stringari, 1999, Rev. Mod. Phys. 71, 463.

Dalgarno, A., and M. R. H. Rudge, 1965, Proc. Roy. Soc. (London) A 286, 519 .

Dalibard, J., 1999, in Bose-Einstein Condensation in Atomic Gases, edited by M. Inguscio, S. Stringari, and C. E. Wieman, International School of Physics Enrico Fermi (IOS Press, Amsterdam), p. 463.

Davis, K. B., M.-O. Mewes, M. R. Andrews, N. J. van Druten, D. S. Durfee, D. M. Kurn, and W. Ketterle, 1995, Phys. Rev. Lett. 75, 3969.

Demkov, Y. N., and V. I. Osherov, 1968, Sov. Phys. - JETP 26, 916.

Derevianko, A., W. R. Johnson, M. S. Safronova, and J. F. Babb, 1999, Phys. Rev. Lett. 82, 3589.

Donley, E. A., N. R. Claussen, S. L. Cornish, J. L. Roberts, E. A. Cornell, and C. E. Wieman, 2001, Nature (London) 412, 295.

Donley, E. A., N. R. Claussen, S. T. Thompson, and C. E. Wieman, 2002, Nature (London) 417, 529.

Doyle, J., B. Friedrich, R. V. Krems, and F. Masnou-Seeuws, 2004, Eur. Phys. J. D 31, 149.

Drummond, P. D., and K. V. Kheruntsyan, 2004, Phys. Rev. A 70, 033609.

Drummond, P. D., K. V. Kheruntsyan, and H. He, 1998, Phys. Rev. Lett. 81, 3055.

Duine, R. A., and H. T. C. Stoof, 2003a, Phys. Rev. A 68, 013602.

Duine, R. A., and H. T. C. Stoof, 2003b, Phys. Rev. Lett. 91, 150405.

Duine, R. A., and H. T. C. Stoof, 2004, Phys. Rep. 396, 115.

Dürr, S., T. Volz, A. Marte, and G. Rempe, 2004a, Phys. Rev. Lett. 92, 020406.

Dürr, S., T. Volz, and G. Rempe, 2004b, Phys. Rev. A 70, 031601(R).

Dürr, S., T. Volz, N. Syassen, G. Rempe, E. van Kempen, S. Kokkelmans, B. Verhaar, and H. Friedrich, 2005, Phys. Rev. A 72, 052707.

Eagles, D. M., 1969, Phys. Rev. 186, 456.

Eckart, C., 1930, Rev. Mod. Phys. 2, 305.

Efimov, V., 1970, Phys. Lett. 33, B 563.

Efimov, V. N., 1971, Sov. J. Nucl. Phys. 12, 589.
Einstein, A., 1924, Sitzungsberichte der Preussischen Akademie der Wissenschaften 22, 261.

Einstein, A., 1925, Sitzungsberichte der Preussischen Akademie der Wissenschaften 1, 3.

Esry, B. D., C. H. Greene, and J. P. Burke, 1999, Phys. Rev. Lett. 83, 1751.

Fano, U., 1935, Nuovo Cimento 12, 154, translation edited by G. Pupillo, A. Zannoni, and C. W. Clark, e-print cond-mat/0502210.

Fano, U., 1961, Phys. Rev. 124, 1866.

Fatemi, F. K., K. M. Jones, and P. D. Lett, 2000, Phys. Rev. Lett. 85, 4462.

Fedichev, P. O., Y. Kagan, G. V. Shlyapnikov, and J. T. M. Walraven, 1996, Phys. Rev. Lett. 77, 2913.

Feshbach, H., 1958, Ann. Phys. (NY) 5, 357.

Feshbach, H., 1962, Ann. Phys. (NY) 19, 287.

Fetter, A. L., and J. D. Walecka, 1971, Quantum theory of manyparticle systems (McGraw-Hill, New York).

Fioretti, A., D. Comparat, A. Crubellier, O. Dulieu, F. MasnouSeeuws, and P. Pillet, 1998, Phys. Rev. Lett. 80, 4402.

Flambaum, V. V., G. F. Gribakin, and C. Harabati, 1999, Phys. Rev. A 59, 1998.

Folman, R., P. Krüger, J. Schmiedmayer, J. Denschlag, and C. Henkel, 2002, Adv. Atom. Mol. Opt. Phys. 48, 263.

Fricke, J., 1996, Ann. Phys. 252, 479.

Friedberg, R., and T. D. Lee, 1989, Phys. Rev. B 40, 6745.

Gao, B., 1996, Phys. Rev. A 54, 2022.

Gao, B., 1998a, Phys. Rev. A 58, 4222.

Gao, B., 1998b, Phys. Rev. A 58, 1728.

Gao, B., 2004, J. Phys. B 37, 4273.

Gao, B., E. Tiesinga, C. J. Williams, and P. S. Julienne, 2005, Phys. Rev. A 72, 042719.

Gasenzer, T., J. Berges, M. G. Schmidt, and M. Seco, 2005, Phys. Rev. A 72, 063604.

Góral, K., M. Gajda, and K. Rzążewski, 2001, Phys. Rev. Lett. 86, 1397.

Góral, K., T. Köhler, and K. Burnett, 2005, Phys. Rev. A 71, 023603.

Góral, K., T. Köhler, S. A. Gardiner, E. Tiesinga, and P. S. Julienne, 2004, J. Phys. B 37, 3457.

Góral, K., L. Santos, and M. Lewenstein, 2002, Phys. Rev. Lett. 88, 170406.

Greiner, M., C. A. Regal, and D. S. Jin, 2003, Nature (London) 426, 537.

Gribakin, G. F., and V. V. Flambaum, 1993, Phys. Rev. A 48, 546.

Grisenti, R. E., W. Schöllkopf, J. P. Toennies, G. C. Hegerfeldt, T. Köhler, and M. Stoll, 2000, Phys. Rev. Lett. 85, 2284.

Gross, E. P., 1961, Nuovo Cimento 20, 454.

Haque, M., and H. T. C. Stoof, 2005, Phys. Rev. A 71, 063603.

Herbig, J., T. Kraemer, M. Mark, T. Weber, C. Chin, H.-C. Nägerl, and R. Grimm, 2003, Science 301, 1510.

Hinds, E. A., C. J. Vale, and M. G. Boshier, 2001, Phys. Rev. Lett. 86, 1462.

Hodby, E., S. T. Thompson, C. A. Regal, M. Greiner, A. C. Wilson, D. S. Jin, E. A. Cornell, and C. E. Wieman, 2005, Phys. Rev. Lett. 94, 120402.

Holland, M., J. Park, and R. Walser, 2001, Phys. Rev. Lett. 86, 1915. Houbiers, M., H. T. C. Stoof, W. I. McAlexander, and R. G. Hulet, 1998, Phys. Rev. A 57, R1497.

Hudson, J. J., B. E. Sauer, M. R. Tarbutt, and E. A. Hinds, 2002, Phys. Rev. Lett. 89, 023003.

Inouye, S., M. R. Andrews, J. Stenger, H.-J. Miesner, D. M. StamperKurn, and W. Ketterle, 1998, Nature (London) 392, 151.

Inouye, S., J. Goldwin, M. L. Olsen, C. Ticknor, J. L. Bohn, and D. S. Jin, 2004, Phys. Rev. Lett. 93, 183201.

Ishkhanyan, A., M. Mackie, A. Carmichael, P. L. Gould, and J. Ja- 
vanainen, 2004, Phys. Rev. A 69, 043612.

Jaksch, D., V. Venturi, J. I. Cirac, C. J. Williams, and P. Zoller, 2002, Phys. Rev. Lett. 89, 040402.

Javanainen, J., M. Kostrun, Y. Zheng, A. Carmichael, U. Shrestha, P. J. Meinel, M. Mackie, O. Dannenberg, and K.-A. Suominen, 2004, Phys. Rev. Lett. 92, 200402.

Jensen, A. S., K. Riisager, D. V. Fedorov, and E. Garrido, 2004, Rev. Mod. Phys. 76, 215.

Jochim, S., M. Bartenstein, A. Altmeyer, G. Hendl, C. Chin, J. H. Denschlag, and R. Grimm, 2003a, Phys. Rev. Lett. 91, 240402.

Jochim, S., M. Bartenstein, A. Altmeyer, G. Hendl, S. Riedl, C. Chin, J. H. Denschlag, and R. Grimm, 2003b, Science 302, 2101.

Julienne, P. S., and F. H. Mies, 1989, J. Opt. Soc. Am. B 6, 2257.

Julienne, P. S., E. Tiesinga, and T. Köhler, 2004, J. Mod. Opt. 51, 1787.

Kagan, Y., B. V. Svistunov, and G. V. Shlyapnikov, 1985, JETP Lett. 42, 209.

Keldysh, L. V., 1965, JETP 20, 1018.

van Kempen, E. G. M., S. J. J. M. F. Kokkelmans, D. J. Heinzen, and B. J. Verhaar, 2002, Phys. Rev. Lett. 88, 093201.

van Kempen, E. G. M., and B. J. Verhaar, 2004, private communication, quoted by S. Dürr et al. (Dürr et al., 2004a).

Kerman, A. J., J. M. Sage, S. Sainis, T. Bergeman, and D. DeMille, 2004, Phys. Rev. Lett. 92, 033004.

Kharchenko, P., J. F. Babb, and A. Dalgarno, 1997, Phys. Rev. A 55, 3566.

Kinast, J., S. L. Hemmer, M. E. Gehm, A. Turlapov, and J. E. Thomas, 2004, Phys. Rev. Lett. 92, 150402.

Köhler, T., and K. Burnett, 2002, Phys. Rev. A 65, 033601.

Köhler, T., T. Gasenzer, and K. Burnett, 2003a, Phys. Rev. A 67, 013601.

Köhler, T., T. Gasenzer, P. S. Julienne, and K. Burnett, 2003b, Phys. Rev. Lett. 91, 230401.

Köhler, T., K. Góral, and T. Gasenzer, 2004, Phys. Rev. A 70, 023613.

Köhler, T., E. Tiesinga, and P. S. Julienne, 2005, Phys. Rev. Lett. 94, 020402.

Kokkelmans, S. J. J. M. F., 2002, private communication, quoted by E. A. Donley et al. (Donley et al., 2002).

Kokkelmans, S. J. J. M. F., and M. J. Holland, 2002, Phys. Rev. Lett. 89, 180401.

Kokkelmans, S. J. J. M. F., J. N. Milstein, M. L. Chiofalo, R. Walser, and M. J. Holland, 2002, Phys. Rev. A 65, 053617.

Kokkelmans, S. J. J. M. F., G. V. Shlyapnikov, and C. Salomon, 2004, Phys. Rev. A 69, 031602(R).

Kotochigova, S., E. Tiesinga, and P. S. Julienne, 2001, Phys. Rev. A 63, 012517.

Kraemer, T., M. Mark, P. Waldburger, J. G. Danzl, C. Chin, B. Engeser, A. D. Lange, K. Pilch, A. Jaakkola, H.-C. Nägerl, and R. Grimm, 2006, Nature (London) 440, 315.

Landau, L. D., 1932, Phys. Z. der Sowjetunion 2, 46.

Leggett, A. J., 1980, in Modern Trends in the Theory of Condensed Matter, edited by A. Pekalski and R. Przystawa (Springer-Verlag, Berlin), p. 13.

Leo, P. J., C. J. Williams, and P. S. Julienne, 2000, Phys. Rev. Lett. 85, 2721.

Loftus, T., C. Regal, C. Ticknor, J. Bohn, and D. Jin, 2002, Phys. Rev. Lett. 88, 173201.

Long, R., T. Steinmetz, P. Hommelhoff, W. Hänsel, T. W. Hänsch, and J. Reichel, 2003, Phil. Trans. R. Soc. Lond. A 361, 1375.

Lovelace, C., 1964, Phys. Rev. 135, B1225.

Luo, F., G. C. McBane, G. Kim, C. F. Giese, and W. R. Gentry, 1993, J. Chem. Phys. 98, 3564.

Luttinger, J. M., and J. C. Ward, 1960, Phys. Rev. 118, 1417.
Macek, J. H., and M. J. Cavagnero, 1998, Phys. Rev. A 58, 348.

Mackie, M., A. Carmichael, M. Kostrun, R. J. Perkins, C. Xu, Y. Zhen, K.-A. Suominen, and J. Javanainen, 2002a, Rapid Adiabatic Passage from an Atomic to a Molecular Condensate, e-print physics/0210131.

Mackie, M., K.-A. Suominen, and J. Javanainen, 2002b, Phys. Rev. Lett. 89, 180403.

Marcelis, B., E. G. M. van Kempen, B. J. Verhaar, and S. J. J. M. F. Kokkelmans, 2004, Phys. Rev. A 70, 012701.

Mark, M., T. Kraemer, J. Herbig, C. Chin, H.-C. Nägerl, and R. Grimm, 2005, Europhys. Lett. 69, 706.

Marte, A., T. Volz, J. Schuster, S. Dürr, G. Rempe, E. G. M. van Kempen, and B. J. Verhaar, 2002, Phys. Rev. Lett. 89, 283202.

Matsubara, T., 1955, Prog. Theor. Phys. (Kyoto) 14, 351.

Mies, F. H., P. S. Julienne, C. J. Williams, and M. Krauss, 1996, J. Res. Natl. Inst. Stand. Technol. 101, 521.

Mies, F. H., and M. Raoult, 2000, Phys. Rev. A 62, 012708.

Mies, F. H., and M. Raoult, 2004, Phys. Rev. A 70, 012710.

Mies, F. H., E. Tiesinga, and P. S. Julienne, 2000, Phys. Rev. A 61, 022721 .

Mitra, A. N., 1962, Phys. Rev. 127, 1342.

Moerdijk, A. J., B. J. Verhaar, and A. Axelsson, 1995, Phys. Rev. A 51, 4852.

Moritz, H., T. Stöferle, K. Günter, M. Köhl, and T. Esslinger, 2005, Phys. Rev. Lett. 94, 210401.

Mukaiyama, T., J. R. Abo-Shaeer, K. Xu, J. K. Chin, and W. Ketterle, 2003, Phys. Rev. Lett. 92, 180402.

Müller, D., D. Z. Anderson, R. J. Grow, P. D. D. Schwindt, and E. A. Cornell, 1999, Phys. Rev. Lett. 83, 5194.

Naidon, P., and F. Masnou-Seeuws, 2003, Phys. Rev. A 68, 033612.

Naidon, P., and F. Masnou-Seeuws, 2006, Phys. Rev. A 73, 043611.

Nielsen, E., and J. H. Macek, 1999, Phys. Rev. Lett. 83, 1566.

Nikolov, A. N., E. E. Eyler, X. T. Wang, J. Li, H. Wang, W. C. Stwalley, and P. L. Gould, 1999, Phys. Rev. Lett. 82, 703.

Nozières, P., and S. Schmitt-Rink, 1985, J. Low Temp. Phys. 59, 195.

Nygaard, N., B. I. Schneider, and P. S. Julienne, 2006, Phys. Rev. A 73, 042705.

O'Hara, K. M., S. L. Hemmer, M. E. Gehm, S. R. Granade, and J. E. Thomas, 2002, Science 298, 2179.

Orso, G., L. P. Pitaevskii, S. Stringari, and M. Wouters, 2005, Phys. Rev. Lett. 95, 060402.

Parish, M. M., B. Mihaila, B. D. Simons, and P. B. Littlewood, 2005, Phys. Rev. Lett. 94, 240402.

Partridge, G. B., K. E. Strecker, R. I. Kamar, M. W. Jack, and R. G. Hulet, 2005, Phys. Rev. Lett. 95, 020404.

Pauling, L., 1939, The nature of the chemical bond and the structure of molecules and crystals (Cornell University Press, Ithaca, NY).

Pazy, E., I. Tikhonenkov, Y. B. Band, M. Fleischhauer, and A. Vardi, 2005, Phys. Rev. Lett. 95, 170403.

Pazy, E., A. Vardi, and Y. B. Band, 2004, Phys. Rev. Lett. 93, 120409.

Perali, A., P. Pieri, and G. C. Strinati, 2005, Phys. Rev. Lett. 95, 010407.

Petrov, D. S., 2004, Phys. Rev. Lett. 93, 143201.

Petrov, D. S., C. Salomon, and G. V. Shlyapnikov, 2004, Phys. Rev. Lett. 93, 090404.

Petrov, D. S., C. Salomon, and G. V. Shlyapnikov, 2005a, J. Phys. B 38, S645.

Petrov, D. S., C. Salomon, and G. V. Shlyapnikov, 2005b, Phys. Rev. A 71, 012708.

Pitaevskii, L. P., 1961, Sov. Phys. - JETP 13, 451.

Proukakis, N. P., K. Burnett, and H. T. C. Stoof, 1998, Phys. Rev. A 57, 1230.

Randeria, M., 1995, in Bose-Einstein Condensation, edited by A. Griffin, D. W. Snoke, and S. Stringari (Cambridge University 
Press, Cambridge), p. 355.

Ranninger, J., and S. Robaszkiewicz, 1985, Physica B+C 135, 468.

Regal, C. A., M. Greiner, and D. S. Jin, 2004a, Phys. Rev. Lett. 92, 083201.

Regal, C. A., M. Greiner, and D. S. Jin, 2004b, Phys. Rev. Lett. 92, 040403.

Regal, C. A., M. Greiner, and D. S. Jin, 2004c, Phys. Rev. Lett. 92, 040403.

Regal, C. A., and D. S. Jin, 2003, Phys. Rev. Lett. 90, 230404.

Regal, C. A., C. Ticknor, J. L. Bohn, and D. S. Jin, 2003a, Nature (London) 424, 47.

Regal, C. A., C. Ticknor, J. L. Bohn, and D. S. Jin, 2003b, Phys. Rev. Lett. 90, 053201.

Rey, A. M., B. L. Hu, E. Calzetta, and C. W. Clark, 2005, Phys. Rev. A 72, 023604

Rice, O. K., 1933, J. Chem. Phys. 1, 375.

Roberts, J. L., N. R. Claussen, J. P. Burke, C. H. Greene, E. A. Cornell, and C. E. Wieman, 1998, Phys. Rev. Lett. 81, 5109.

Roberts, J. L., N. R. Claussen, S. L. Cornish, E. A. Donley, E. A. Cornell, and C. E. Wieman, 2000a, Phys. Rev. Lett. 86, 4211.

Roberts, J. L., N. R. Claussen, S. L. Cornish, and C. E. Wieman, 2000b, Phys. Rev. Lett. 85, 728.

Sage, J. M., S. Sainis, T. Bergeman, and D. DeMille, 2005, Phys. Rev. Lett. 94, 203001.

Sandars, P. G. H., 1975, in Atomic Physics 4, edited by G. zu Putlitz (Plenum, New York), p. 71.

Schöllkopf, W., and J. P. Toennies, 1994, Science 266, 1345.

Schrieffer, J. R., 1964, Theory of Superconductivity (W. A. Benjamin, New York).

Schunck, C. H., M. W. Zwierlein, C. A. Stan, S. M. F. Raupach, W. Ketterle, A. Simoni, E. Tiesinga, C. J. Williams, and P. S. Julienne, 2005, Phys. Rev. A 71, 045601.

Schwinger, J., 1947a, Harvard lecture notes, quoted by H. A. Bethe (Bethe, 1949).

Schwinger, J., 1947b, Phys. Rev. 72, 738.

Schwinger, J., 1961, J. Math. Phys. 2, 407.

Simonucci, S., P. Pieri, and G. C. Strinati, 2005, Europhys. Lett. 69, 713.

Stan, C. A., M. W. Zwierlein, C. H. Schunck, S. M. F. Raupach, and W. Ketterle, 2004, Phys. Rev. Lett. 93, 143001.

Stenger, J., S. Inouye, M. R. Andrews, H.-J. Miesner, D. M. StamperKurn, and W. Ketterle, 1999, Phys. Rev. Lett. 82, 2422.

Stoll, M., and T. Köhler, 2005, Phys. Rev. A 72, 022714.

Stoof, H. T. C., A. M. L. Janssen, J. M. V. A. Koelman, and B. J. Verhaar, 1989, Phys. Rev. A 39, 3157.

Stoof, H. T. C., J. M. V. A. Koelman, and B. J. Verhaar, 1988, Phys. Rev. B 38, 4688.

Strecker, K. E., G. B. Partridge, and R. G. Hulet, 2003, Phys. Rev. Lett. 91, 080406.

Stwalley, W. C., 1976, Phys. Rev. Lett. 37, 1628.

Szymańska, M. H., K. Góral, T. Köhler, and K. Burnett, 2005, Phys. Rev. A 72, 013610.

Takekoshi, T., B. M. Patterson, and R. J. Knize, 1999, Phys. Rev. A 59, R5.

Tang, K. T., J. P. Toennies, and C. L. Yiu, 1995, Phys. Rev. Lett. 74, 1546.

Taylor, J. R., 1972, Scattering Theory (Wiley, New York).

Thalhammer, G., K. Winkler, F. Lang, S. Schmid, R. Grimm, and J. H. Denschlag, 2006, Phys. Rev. Lett. 96, 050402.

Theis, M., G. Thalhammer, K. Winkler, M. Hellwig, G. Ruff, R. Grimm, and J. H. Denschlag, 2004, Phys. Rev. Lett. 93, 123001.

Thompson, S., E. Hodby, and C. Wieman, 2005a, Phys. Rev. Lett. 95, 190404.
Thompson, S. T., E. Hodby, and C. E. Wieman, 2005b, Phys. Rev. Lett. 94, 020401.

Thywissen, J. H., R. M. Westervelt, and M. Prentiss, 1999, Phys. Rev. Lett. 83, 3762.

Tiesinga, E., B. J. Verhaar, and H. T. C. Stoof, 1993, Phys. Rev. A 47, 4114.

Tiesinga, E., C. J. Williams, F. H. Mies, and P. S. Julienne, 2000, Phys. Rev. A 61, 063416.

Timmermans, E., P. Tommasini, R. Côté, M. Hussein, and A. Kerman, 1998, Inter-Condensate Tunneling in Bose-Einstein Condensates with Feshbach Resonances, e-print cond-mat/9805323.

Timmermans, E., P. Tommasini, R. Côté, M. Hussein, and A. Kerman, 1999a, Phys. Rev. Lett. 83, 2691.

Timmermans, E., P. Tommasini, M. Hussein, and A. Kerman, 1999b, Phys. Rep. 315, 199.

Tommasini, P., E. Timmermans, M. Hussein, and A. Kerman, 1998, Feshbach Resonance and Hybrid Atomic/Molecular BECSystems, e-print cond-mat/9804015.

Uang, Y. H., R. F. Ferrante, and W. C. Stwalley, 1981, J. Chem. Phys. 74, 6267.

Uang, Y.-H., and W. C. Stwalley, 1980, Phys. Rev. Lett. 45, 627.

Uehling, E. A., and G. E. Uhlenbeck, 1933, Phys. Rev. 43, 552.

Volz, T., S. Dürr, S. Ernst, A. Marte, and G. Rempe, 2003, Phys. Rev. A 68, 010702(R).

Volz, T., S. Dürr, N. Syassen, G. Rempe, E. van Kempen, and S. Kokkelmans, 2005, Phys. Rev. A 72, 010704.

Vuletic, V., A. J. Kerman, C. Chin, and S. Chu, 1998, Phys. Rev. Lett. 82, 1406.

Wang, D., J. Qi, M. F. Stone, O. Nikolayeva, H. Wang, B. Hattaway, S. D. Gensemer, P. L. Gould, E. E. Eyler, and W. C. Stwalley, 2004, Phys. Rev. Lett. 93, 243005.

Weber, T., J. Herbig, M. Mark, H.-C. Nägerl, and R. Grimm, 2003, Science 299, 232.

Weinberg, S., 1996, The Quantum Theory of Fields (Cambridge University Press, Cambridge, England).

Weiner, J., V. S. Bagnato, S. Zilio, and P. S. Julienne, 1999, Rev. Mod. Phys. 71, 1.

Weinstein, J. D., and K. G. Libbrecht, 1995, Phys. Rev. A 52, 4004.

Wick, G. C., 1950, Phys. Rev. 80, 268.

Wigner, E. P., 1927, Z. Phys. 43, 624.

Wigner, E. P., 1948, Phys. Rev. 73, 1002.

Williams, J. E., T. Nikuni, N. Nygaard, and C. W. Clark, 2004a, J. Phys. B 37, 351.

Williams, J. E., N. Nygaard, and C. W. Clark, 2004b, New J. Phys. 6, 123 .

Williams, J. E., N. Nygaard, and C. W. Clark, 2006, New J. Phys. 8, 150.

Wouters, M., and G. Orso, 2006, Phys. Rev. A 73, 012707.

Wynar, R., R. S. Freeland, D. J. Han, C. Ryu, and D. J. Heinzen, 2000, Science 287, 1016.

Xu, K., T. Mukaiyama, J. R. Abo-Shaeer, J. K. Chin, D. E. Miller, and W. Ketterle, 2003, Phys. Rev. Lett. 91, 210402.

Yamaguchi, Y., 1954, Phys. Rev. 95, 1628.

Yan, Z.-C., J. F. Babb, A. Dalgarno, and G. W. F. Drake, 1996, Phys. Rev. A 54, 2824.

Yurovsky, V. A., and A. Ben-Reuven, 1998, J. Phys. B 31, 1.

Yurovsky, V. A., and A. Ben-Reuven, 2003a, J. Phys. B 36, 335.

Yurovsky, V. A., and A. Ben-Reuven, 2003b, Phys. Rev. A 67, 043611.

Yurovsky, V. A., and A. Ben-Reuven, 2004, Phys. Rev. A 70, 013613. Yurovsky, V. A., and A. Ben-Reuven, 2005, Phys. Rev. A 72, 053618. Yurovsky, V. A., A. Ben-Reuven, P. S. Julienne, and Y. B. Band, 1999a, J. Phys. B 32, 1845.

Yurovsky, V. A., A. Ben-Reuven, P. S. Julienne, and C. J. Williams, 
1999b, Phys. Rev. A 60, R765.

Yurovsky, V. A., A. Ben-Reuven, P. S. Julienne, and C. J. Williams, 2000, Phys. Rev. A 62, 043605.

Zener, C., 1932, Proc. Roy. Soc. London Ser. A 137, 696.

Zhang, J., E. G. M. van Kempen, T. Bourdel, L. Khaykovich, J. Cubizolles, F. Chevy, M. Teichmann, L. Tarruell, S. J. J. M. F. Kokkelmans, and C. Salomon, 2004, Phys. Rev. A 70, 030702(R).

Zoller, P., 2002, Nature (London) 417, 493.
Zwierlein, M. W., J. R. Abo-Shaeer, A. Schirotzek, C. H. Schunck, and W. Ketterle, 2005, Nature 435, 1047.

Zwierlein, M. W., C. A. Stan, C. H. Schunck, S. M. F. Raupach, S. Gupta, Z. Hadzibabic, and W. Ketterle, 2003, Phys. Rev. Lett. 91, 250401.

Zwierlein, M. W., C. A. Stan, C. H. Schunck, S. M. F. Raupach, A. J. Kerman, and W. Ketterle, 2004, Phys. Rev. Lett. 92, 120403. 